\title{
ICC 2002 Innovative Confinement Concepts
}

\author{
E.B. Hooper
}

January 15, 2002

U.S. Department of Energy

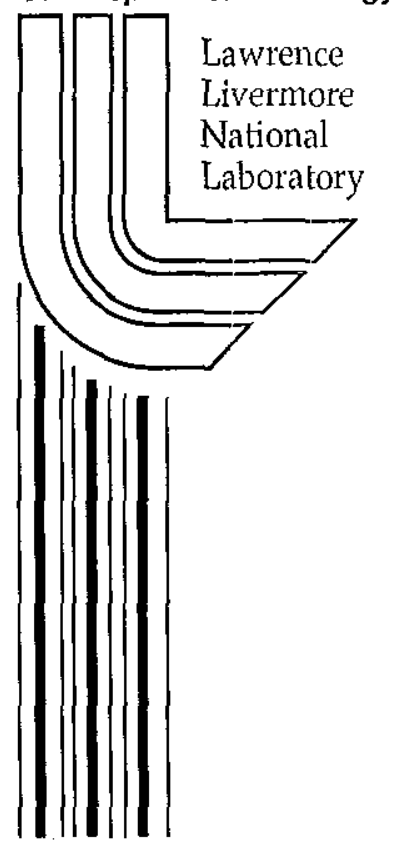





\section{DISCLAIMER}

This document was prepared as an account of work sponsored by an agency of the United States Government. Neither the United States Government nor the University of California nor any of their employees, makes any warranty, express or implicd, or assumes any legal liability or responsibility for the accuracy, completeness, or usefulness of any information, apparatus, product, or process disclosed, or represents that its use would not infringe privately owned rights. Reference herein to any specific commercial product, process, or service by trade name, trademark, manufacturer, or otherwise, does not necessarily constitute or imply its endorsement, recommendation, or favoring by the United States Government or the University of California. The views and opinions of authors expressed herein do not necessarily state or reflect those of the United States Government or the University of California, and shall not be used for advertising or product endorsement purposes.

This work was performed under the auspices of the U. S. Department of Energy by the University of California, Lawrence Livermore National Laboratory under Contract No. W-7405-Eng-48.

This report has been reproduced directly from the best available copy.

Available electronically at http://www.doc.gov/bridge

Available for a processing fee to U.S. Department of Energy

And its contractors in paper from

U.S. Department of Energy

Office of Scientific and Technical Information

P.O. Box 62

Oak Ridge, TN 37831-0062

Telephone: (865) 576-8401

Facsimile: (865) $576-5728$

E-mail: reports@adonis.osti.gov

Available for the sale to the public from

U.S. Department of Commerce

National Technical Information Service

5285 Port Royal Road

Springfield, VA 22161

Telephone: (800) 553-6847

Facsimile: (703) 605-6900

E-mail: orders@ntis.fedworld.gov

Online ordering: http://www.ntis.gov/ordering.htm

OR

Lawrence Livermore National Laboratory

Technical Information Department's Digital Library

http://www.llnl.gov/tid/Library.html 



\section{$1 \mathrm{CCC} 2002$ \\ Hnnovative $\mathbb{C}$ onn finnement Concepts}

January 22-24, 2002

University of Maryland, College Park
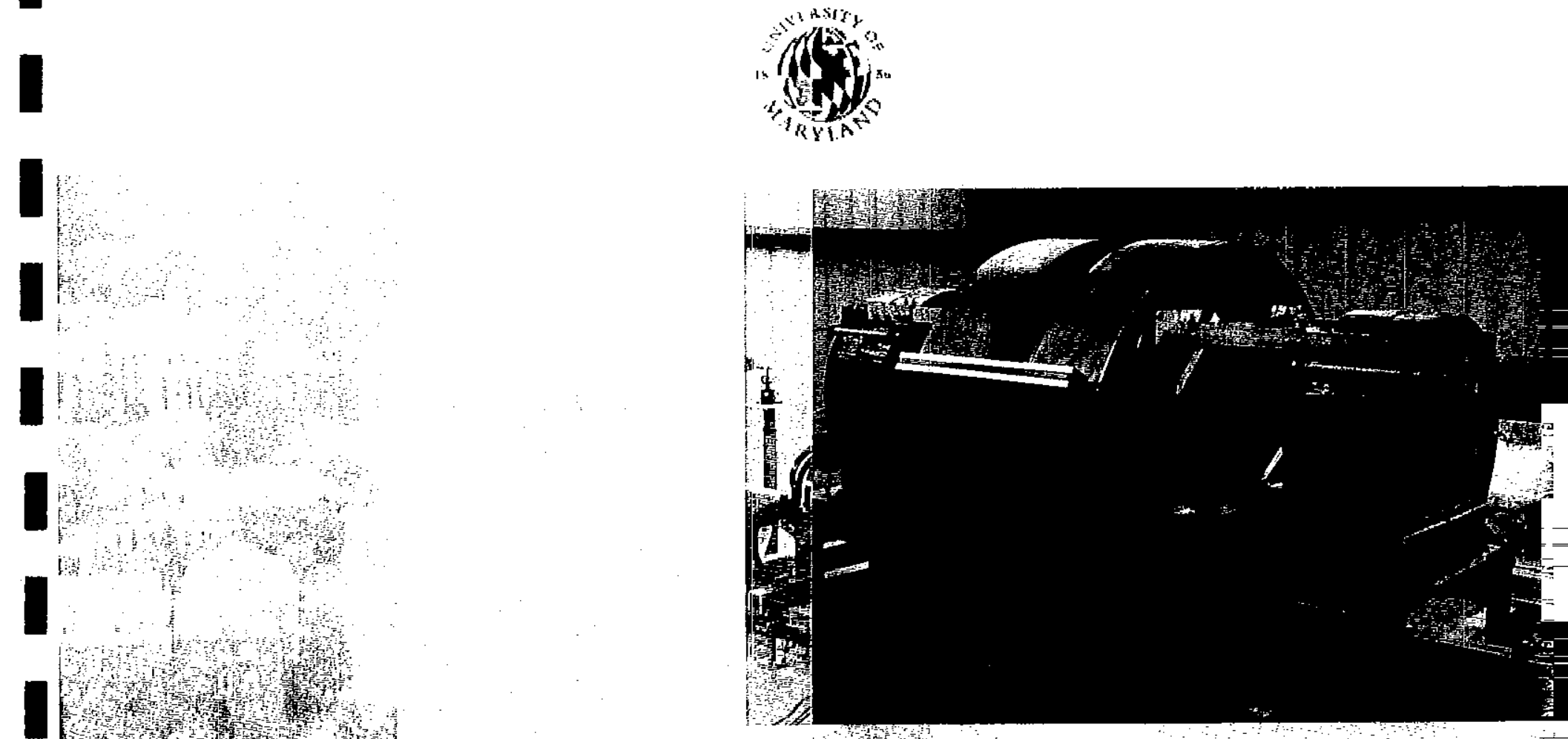

Maryland Centrifugal Experiment, MCX, jan 2002

Whlute Stabilization in MCX, simulation, R- $\phi$ plane Fin
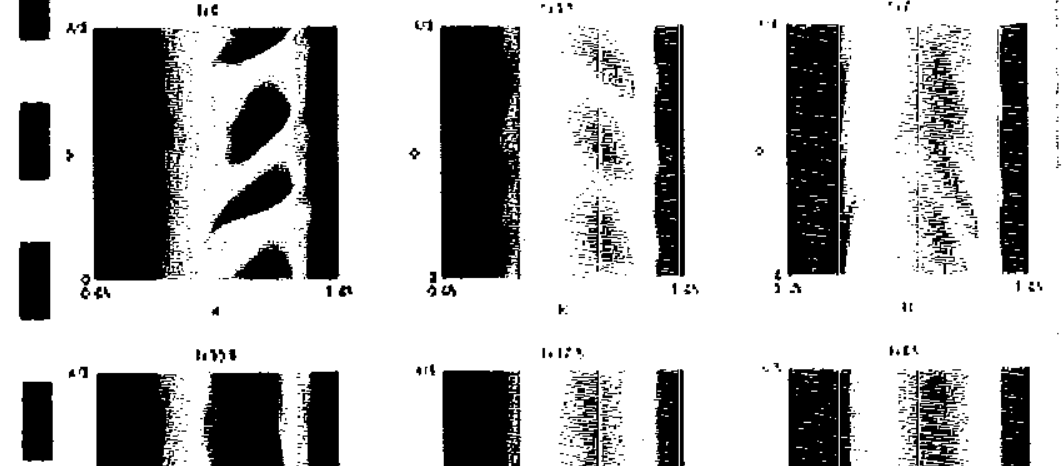

ine
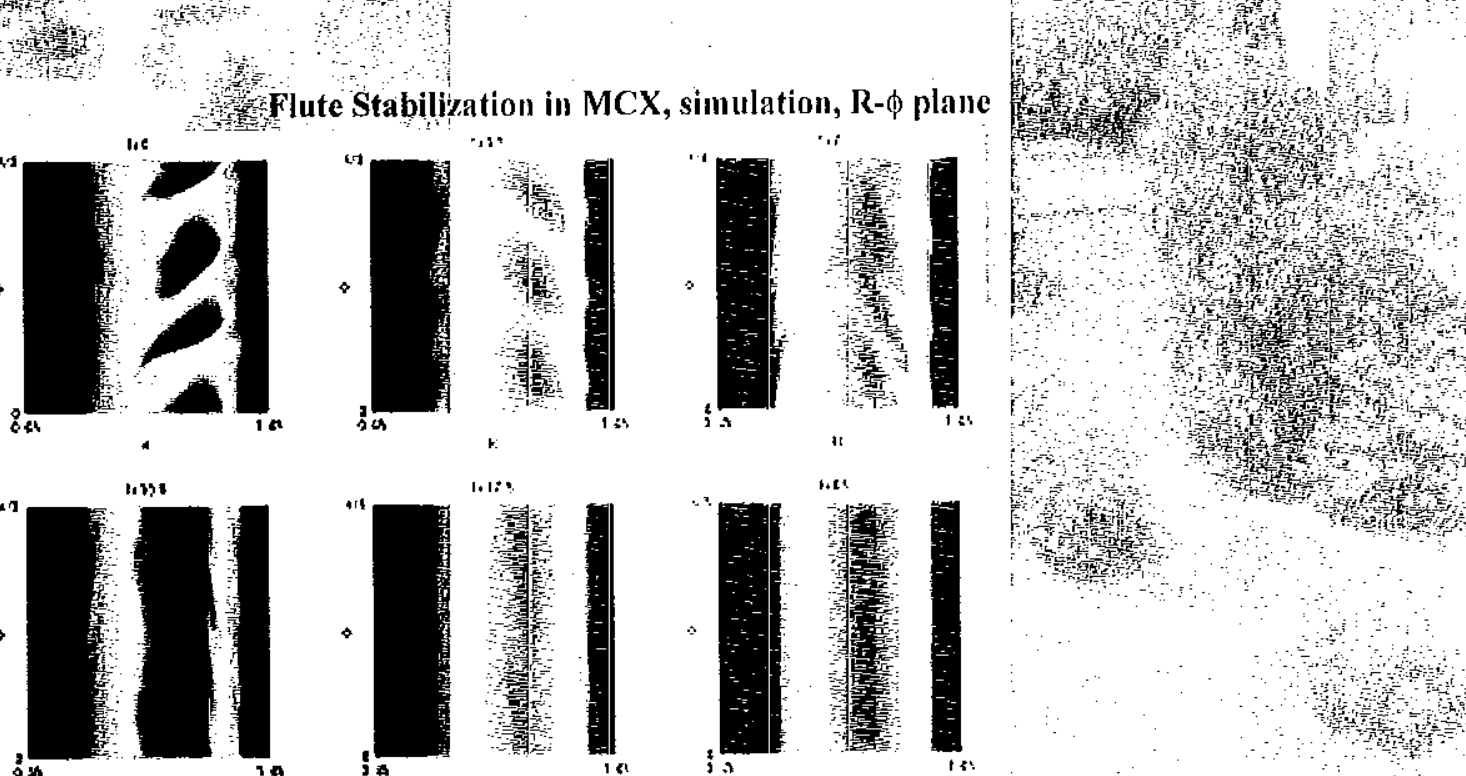
The program, abstracts, etc. are also available at https:/ / wormhole.ucllnl.org/ICC2002/

\section{ACKNOWLEDGEMENTS}

The Program and Local Committees gratefully acknowledge the hard work contributed by Bill Meyer, Web Master, and Gloria Davalos, Administrator. The high quality organization of this conference is due entirely to their effort. 


\section{Program Committee:}

David Anderson, University of Wisconsin

John Greenley, Cornell University

Rob Goldston, Princeton Plasma Physics Laboratory

Cris Hegna, University of Wisconsin

William Heidbrink, University of California, Irvine

Alan Hoffman, University of Washington

Bick Hooper, Lawrence Livermore National Laboratory

Steve Jardin, Princeton Plasma Physics Laboratory

Jay Kesner, MIT Plasma Science and Fusion Center

Ron Kirkpatrick, Los Alamos National Laboratory

Grant Logan, Lawrence Berkeley National Laboratory

Jim Lyon, Oak Ridge National Laboratory

Jerry Navratil, Columbia University

Martin Peng, Oak Ridge National Laboratory

John Perkins, Lawrence Livermore National Laboratory

Stewart Prager, University of Wisconsin

John Sarff, University of Wisconsin

Mike Schaffer, General Atomics

Kurt Schoenberg, Los Alamos National Laboratory

Bob Taylor, University of California, Los Angeles

George Tynan, University of California, San Diego

Mike Zarnstroff, Princeton Plasma Physics Laboratory

\section{Local Committee:}

Rick Ellis, University of Maryland Adil Hassam, University of Maryland 
7

r
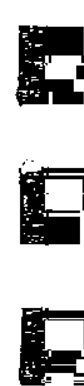

$\equiv$

$r=$

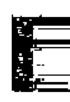

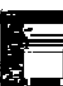




\section{ICC2002 Agenda}

Tuesday, January 22, 2002

8:15 Setup posters for Tuesday's talks

8:30 Welcome, Administrative items

Oral Session 1 (Kurt Schoenberg, chair)

$8: 45$

$9: 10$

$9: 15$

$9: 40$

$9: 45$

10:10

$10: 15$

Stewart Prager
Discussion

Advances in Energy Continement in the Reversed Field Pinch

David Hill

Discussion

Alan Hoffman

Overview of Recent Fiesults from the SSPX Spheromak Experiment

Discussion

Field Reversed Configurations - Issues, Status, and Plans

Break

$10: 30$

$10: 55$

11:00

$11: 30$

$11: 50$

$11: 55$

Grant Logan

Discussion

Discussion of morning presentations

General Meeting on Breakout Sessions (Skunkworks. Burning Plasma Sclence and the Magnetic ICCs.) (Stewart Prager, Chair)

$12: 00$

Jerry Navratil Snowmass and the ICCs

Discussion

John Perkins $\quad$ Skunkworks

$1: 00$

Lunch

\section{Breakout sessions}

(1) Skunkworks

(2) Burning Plasma: Energetic Particles and Alpha Particles (Raffi Nazikian)

Oral Session 2 (Grant Logan, Chair)

$2: 30$

$2: 55$

3:00

$3: 25$

$3: 30$

$3: 55$

$4: 00$

$4: 30$

Andy Schmitt Laser Inertial Fusion Energy

Discussion

Mike Key

Discussion

Keith Matzen

Fast Ignition

Discussion

Pulsed Power ICF and IFE Research on $Z$ and $Z$ R .

Discussion of afternoon presentations

Poster Session 1 (RFP, spheromak, FRG, HIF, LIF, FI, Pulsed Power)

$6: 15$

End Session 


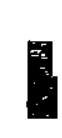

E

E

$\therefore$

-

F

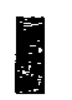

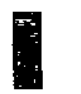

$x$

E

$\Rightarrow$
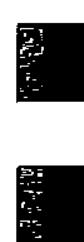

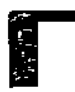

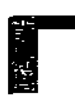

$\approx$

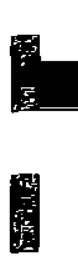


Wednesday, January 23, 2002

$8: 15$

$8: 30$

$8: 55$

9:00

$9: 25$

$9: 30$

9:55

10:00

$10: 25$

$10: 30$

$10: 45$

$11: 15$

$12: 00$

$1: 00$

$2: 30$

$2: 45$

3:10

$3: 15$

$3: 40$

$3: 45$

$4: 15$

$6: 00$

$8: 15$

Setup posters for Wedesday's talks

Oral Session 3 (John Sarf, Chair)

Randy Wilson Physics Progress on the NSTX Device

Discussion

Bob Taylor

ET Results and Burning Plasma Options

Discussion

Steve Knowlton

Stellarator

Discussion

Daren Garnier

Progress in Lovitated Dipole Research

Discussion

Break

Discussion of morning presentations

Breakout sessions

(1) Skunkworks

(2) Burning Plasma: MHD (Ted Strait and Chris Hegna)

Lunch

Breakout Sessions (cont.)

Break

Oral Session 4 (Ron Kirkpatrick Chair)

George Miley Overview of Research and Issues for Ion-Injected IEC Fusion

Discussion

Rick Nebel

Experimental and Theoretical Studies of Electrostatic Confinement

Discussion

Discussion of afternoon presentations

Poster Session 2 (ST, ET, stellarator, IEC)

End Session

Community meeting with OFES 
Thursday, January 24, 2002
$8: 15$
Setup posters for Thursday's talks
Oral Session 5 (Alan Hofiman, Chair)
$8: 30$
$8: 55$
9:00
$9: 25$
$9: 30$
$9: 55$
Dick Siemon
Discussion
An overview of Progress on MTF Research
Uri Shumlak
Evidence of Stabilization in the ZAP Z-pinch Experiment
Discussion
Rick Ellis
MCX
$10: 00$
Break
$10: 15$
Discussion of morning presentations
$10: 45$
Poster Session 3 (MTF, Z-Pinch, MCX)
$12: 15$
Lunch
$1: 15$
Breakout sessions
(1) Skunkworks
(2) Burning Plasma: Edge and Boundary Physics (Spencer Pitcher)
3:00
Summaries of Breakout Sessions
$5: 00$
End Workshop 


\section{Innovative Confinement Concepts \\ January 22, 2002 - January 24, 2002 \\ College Park, Maryland}

\begin{tabular}{|c|c|c|}
\hline Anderson, David & University of Wisconsin & dtanders@facstaff.wisc.edu \\
\hline Barnes, Daniel (Dan) & Los Alamos National Laboratory & dbarnes@lanl.gov \\
\hline Bauer, Bruno & University of Nevada Reno & bbauer@physics.unr.edu \\
\hline Sellan, Paul & Caltech & pbellan@its.caltech.edu \\
\hline Belova, Elena & Princeton Plasma Physics Laboratory & ebelova@pppl.gov \\
\hline \multicolumn{3}{|c|}{ Bernal, Santiago Institute for Research in Electronics and Applied Physics sabern@glue.umd.edu } \\
\hline Bhatt, Ronak & MTT & ronak@mit.edu \\
\hline Bourque, Robert & General Atomics & robert.bourque@gat.com \\
\hline rown, Michael & Swarthmore College & doc@swarthmore.edu \\
\hline ohen, Sam & Princeton Plasma Physics Laboratory & scohen@pppl.gov \\
\hline Commisso, Robert & Naval Research Laboratory & commisso@suzie.nrl.navy.mil \\
\hline Tothran, Christopher & Swarthmore College & ccothra1@swarthmore.edu \\
\hline llis, Richard & University of Maryland & rfellis@glue.umd.edu \\
\hline Fonck, Raymond & University of Wisconsin-Madison & fonck@engr.wisc.edu \\
\hline Garnier, Darren & Columbia University & garnier@psfc.mit.edu \\
\hline Garstka, Gregory & University of Wisconsin-Madison & garstka@engr.wisc.edu \\
\hline Gauvreau, Jean-Luc & UCLA & gauvreau@ucla.edu \\
\hline Goldston, Robert & Princeton Plasma Physics Laboratory & ton@pppl.gov \\
\hline Greenly, John & Cornell University & jbg2@cornell.edu \\
\hline Gretchikha, Alexei & Cor & ag50@cornell.edu \\
\hline Grossmann, William & ALSTOM Power & rossmann@power.alstom.com \\
\hline Grossnickle, Jim & University of Washington & jag@rppl.aa.washin \\
\hline Guo, Houyang & of Washington & @aa.washington.edu \\
\hline Gupta, Deepak & Aaryland & a@glue.umd:edu \\
\hline er, James & nce Livermore National Laboratory & r2@11 \\
\hline , Alexander & ersity & it.edu \\
\hline Hassam, Adil & Univ & has \\
\hline Hawryluk, Richard & Plasma Physics Laboratory & tk@pppl.gov \\
\hline Hill, & e National Laboratory & hill7@llnl.gov \\
\hline Hoffman, Alan & University of Washington ho & hoffman@aawashington.edu \\
\hline Hooper, Bick & Lawrence Livermore National Laboratory & hooper1@llnl.gov \\
\hline Hsu, Scott & Calte & tech.edu \\
\hline Huang, Yi-Min & , University of Maryland & d.edu \\
\hline Jarboe, Thomas & University of $W$ & @aa.washington.edu \\
\hline , Stephen & Princeton Plasma Physics Laboratory & pppl.gov \\
\hline Kesner, Jay & $\mathrm{MT \Gamma}$ & psfc.mit.edu \\
\hline Key, Michael & Lawrence Livermore National Laboratory & Inl.gov \\
\hline Kirkpatrick, Ronald & onal Laboratory & al.gov \\
\hline Knowlton, Stephen & iversity & knowlton@physics.auburn.edu \\
\hline Koloc, Paul & Prometheus II, Ltd. & pmk@plasmak.com \\
\hline Kotschenreuther, Mike & Institute for Fusion Studies & mtk@pauling.ph.utexas.edu \\
\hline Kulsrud, Russell & Princeton Plasma Physics Laboratory & rmk@pppl.gov \\
\hline Lee, Dongwoo & Cornell University & d1228@cornell.edu \\
\hline B. Grant & Lawrence Berkeley National Laboratory & bglogan@lbl.gov \\
\hline & idge National Laboratory & f@ornl.gov \\
\hline Majeski, Dick & Princeton Plasma Physics Laboratory & rmajeski@pppl.gov \\
\hline Makhin, Volodymyr & University of Nevada Reno & makhin@physics.unr.edu \\
\hline Manheimer, Wallace & Naval Research Laboratory & manheime@ccf.nrl.navy.mil \\
\hline
\end{tabular}


Matzen, $\mathrm{M}$.

Mauel, Michael

McCollam, Karsten

Mead, William

Messer, Sarah

Miley, George

Miller, Kenneth

Milroy, Richard

Navratil, Gerald

Nebel, Richard

Neilson, Hutch

O'Connell, Robert

OShea, Patrick

Ono, Masayuki

Ottinger, Paul

Paul, Stephen

Peng, Martin

Perkins, L. John

Peter, Andrew

Plummer, Dirk

Podulka, William

Post, Richard

Prager, Stewart

Presura, Radu

Raman, Roger

Redd, Aaron

Robertson, Scott

Ryutov, Dmitri

Santarius, John

Sarff, John

Schaffer, Michael

Schauer, Martin

Schmitt, Andrew

Schmitz, Lothar

Schoenberg, Kurt

Shumlak, Uri

Sieck, Paul

Siemon, Richard

Simakov, Andrei

Slough, John

Sotnikov, Vladimir

Sovinec, Carl

Spong, Donald

Stallard, Barry

Taccetti, Martin

Taylor, Robert

Thio, Y. C. Francis

Tynan, George

Wilson, James

Wong, H. Vernon

Woodruff, Simon

Wurden, Glen

Zarnstorff, Michael
Sandia National Laboratories

Columbia University

University of Wisconsin

ANSR, Inc.

University of Maryland-College Park

U of IL - NPRE

RPPL, University of Washington

University of Washington

Columbia University

Los Alamos National Laboratory

Princeton Plasma Physics Laboratory

University of Wisconsin - Madison

Univ. of Maryland

Princeton Plasma Physics Lab

Naval Research Laboratory

Princeton Plasma Physics Laboratory

Oak Ridge National Lab (1) PPPL.

Lawrence Livermore National Laboratory

RPPL

Dirk Arnold Plummer, P.E.

Cornell University

Lawrence Livermore National Laboratory

University of Wisconsin-Madison

University of Nevada Reno

University of Washington

The Helicity Injected Torus Experiment

Univ. of Colorado, Dept. Physics

Lawrence Livermore National Laboratory

Univ. of Wisconsin

Univ. of Wisconsin-Madison

General Atomics

Los Alamos National Laboratory

Naval Research Laboratory - Plasma

University of California, Los Angeles

Los Alamos Nat Laboratory

University of Washington

University of Washington

Los Alamos National Laboratory

MIT PSFC

University of Washington

University of Nevada Reno

University of Wisconsin-Madison

Oak Ridge National Laboratory

Lawrence Livermore National Laboratory

Los Alamos National Laboratory

University of California, Los Angees

NASA Marshall Space Flight Ctr

UCSD

Princeton Plasma Physics Lab

University of Texas at Austin

Lawrence Livermore National Laboratory

Los Alamos National Laboratory

Princeton Plasma Physics Laboratory mkmatze@sandia.gov

mauel@columbia.edu

kmccollam@facstaff.wisc.edu

wcm@ansr.com

messer@glue.umd.edu

g-miley@uiuc.edu

kemiller@rppl.aa.washington,edu

rmilroy@aa.washington.edu

navratil@columbia.edu

rnebel@lanl.gov

hneilson@pppl.gov

roconnell@facstaff.wisc.edu

poshea@eng.umd.edu

mono@pppl.gov

ottinger@suzie.nrl.navy.mil

spaul@pppl.gov

mpeng@pppl.gov

perkins3@llnl.gov

peter@aa.washington.edu

dap@alum.mit.edu

bpodul@lps.cornell.edu

post3@Ilnl.gov

scprager@facstaff.wisc.edu

presura@physics.unr.edu

raman@aa.washington.edu

redd@aa.washington.edu

robertso@stripe.colorado.edu

ryutov1@llnl.gov

santarius@engr.wisc.edu

jssarff@facstaff.wisc.edu

schaffer@fusion.gat.com

schauer@lanl.gov

schmitt@this.nrl,mil

1schmitz@ucla.edu

kurts@lanl.gov

shumlak@aa.washington.edu

sieck@aa.washington.edu

siemon@lanl.gov

simakov@mit.edu

slough@aa.washington.edu

sotnikov@physics.unr.edu

sovinec@engr.wisc.edu

spongda@ornl.gov

stallard1@lln.gov

taccetti@lanl.gov

rtay;or@ucla.edu

francis.thio@msfc.nasa.gov

gtynan@ucsd.edu

jrwilson@pppl.gov

navajo@mail.utexas,edu

woodruff7@llnl.gov

wurden@lanl.gov

mzarnstorff@pppl,gov 

Additional posters by Roger D. Bengtson, Priyanka Goswami, Aaron J. Redd, and Carl Sovinec have been included alphabetically in the poster abstract session. 
Bames, D. C, Bames, D. C. Baver, B. S.

Bellan, P. M

Bellan, P. M.

Belova, E.V. Belova, E. V. Bourque, R. F. Brown, M, R. Degnan, J. $\mathrm{H}$.

Ellls, R, F.

Fonck, R. J.

Frese, M. H.

Garnler, D. T.

Garstka, G. D.

Gauvreau, J, L.

Grabowski, T. C.

Greenly, J. B.

Gretchikha, A. V.

Grossnickle, J. A.

Guo, H. Y.

Gupta, D. K.

Hammer, J. $\mathrm{H}$.

Hansen, A. K.

Hassam, A.

Hill, D. N.

Hoffman, A. L.

Hooper, E. B.

Hsu, S. C.

Huang, Y. $M$

Jardln, S. C.

Kotschenreuther, $\mathrm{M}$. Kesner, J.

Key, M. H.

Klrkpatilck, R. C.

Knowlton, S. F.

Knowlton, S.F.

Knyazev, 8 .

Koloc, P. M.

Koloc, P. M.

Kuisrud, R. M

Kussrud, R. M

Logan. B. G,

Logan, B. G.

Lyon, J. F.

Lyon, J. F,

Majeskl, $R$,

Manhelmer, $\mathrm{W}$.

Mauel, M. E.

Matzen, M. K.

McCollam, $K, J$.
FRC Stability Explained?

FRC

Poster 1

1.13

Plasma Heat Engine for High Q Aneutronic Fusion?

Skunkworks

Energy Transport in Wall-Confined Plasmas Relevant to Magnetized MTF

Poster 3

3.05

Target Fusion (MTF)

How initially vacuum flux tubes develop uniform cross-sectlon when Spheromak

Poster 1

1.10

an axial current is made to flow

Fusion via stagnation of self-excited counter-streaming toroldal Skunkworks

flows in a highty rippled tokamak

Kinetic Stabillty of the Fleld-Reversed Configuration

Numerlcal Simulations of Compressional Alfven Modes In NSTX

RMS - Repetitive Merging of Spheromaks

Overview of the SSX-FRC experiment

Comparison of Z-Pinch and Theta-Pinch Drive Suitable for

Compression of FRC Configurations

The Maryland Centrigugal Experiment: Motivation and Status MCX

Performance and Stability Limits at Near-unity Aspect Ratlo In the ST

Pegasus Toroidal Experiment

Field-Reversed Formation Simulations with $M A C H 2 \quad$ FRC

Progress in Levltated Dipole Research LDX

Equilibrium and Stabllity Analysis of Pegasus Plasmas ST

Equllibrlum of the Electric Tokamak

Crowbar Switch for Configuration Formation Field Reversed

lon ring formation by radial injection

Simulations of lon ring dynamics

FRC Translation and Capture Measurements on TCS

Formation and steady state maintenance of FRCs using Rotating

Magnetic Field (RMF) current drive in the TCS

Status of the Maryland Centrlfugal Experiment (MCX) MCX

impact Fusion Revisited

Physics and Operations Plan for LOX

Alfvenically Confined Plasmas

Overview of Recent Results from the SSPX Spheromak Experiment

Fleld-Reversed Configurations - Issues, Status, and Plans

Modeling SSPX Formation and Equilibrio

FRC

Spheromak

FRC

MTF

SC

ST

ET

FRC

FRC

FRC

FRC

Skunkworks

LDX

Skunkworks

Spheromak

FRC

Spheromak

Experimental Study of Spheromak Formation Using a Planar Coaxial Spheromak

NMCX: Numerical Maryland Centrifugal Experiment MCX

SImulation of Inductive Startup of a Spherical Tonus without using an ST

OH Solenoid

Exhausting Plasma to Outside the TF coils

Theory of Plasma Confinement in a Levitated Dipole L.DX

Fast Ignition

Fleld Gradlent Dependence for Fusion Self-Heating in MTF Targets

Stellarator Overview

Planned MHD Stability Studies of Current-Carrying Plasmas In the

Compact Toroidai Hybrid

Spectroscopic Diagnostics of Plasma Response to fon Ring

Plasma Magnetic Confinement: The PLASMAK

The Engineering Physics of an Optimized Confinement Concept The Plasmak)

Muon Catalyzed Fusion Revisited

Muon Catalyzed Fusion Revisited

Heavy ion beam science and innovative technology towards

Inertial fusion energy

Fast lgnition and Heavy-lon Indirect Drive

Physics Overview of the QPS Experiment

Design of the QPS Experiment

A Lithium Wall Tokamak Experiment

Carbon Free Energy Self Sufficlency by 2050? Posslbly Vla the Thorlum/233U Fisslon Fusion Hybrid

Status of LDX Fabrication

Pulsed Power ICF and IFE Research on Z and ZR

Osclitating Field Current Orive in the Madison Symmetrlc Torus
IFE

MTF

Stellarator

Stellarator

FRC

Spheromak

MTF

Misc.

HIF

Skunkworks

Stellarator

Stellarator

ST

LDX

IFE

RFP
Poster 1

Poster 2

Poster I

Poster 1

Poster 3

Oral 5

Poster 2

Poster 1

Oral 3

Poster 2

Poster 2

Poster 3

Poster 1

Poster 1

Poster 1

Poster 1

Poster 3

Posier 2

Oral 1

Oral?

Poster 1

Poster 1

Poster 3

Poster 2

Poster 2

Poster 2

Oral 2

Poster 3

Oral 3

Poster 2

Poster 1

Poster 1

Poster 3

Poster 3

Skunkworks

Oral 1

Poster 2

Poster 2

Poster 2

Skunkworks

Poster 2

Oral 2

Poster 1
2.09

2.10

2.14

0.23

1.14

1.20

3.06

0.53

2.05

1.21

2.06

2.12

1.26

1.15

1.16

3.14

0.12

106

1.09

3.15

2.02

2.22

2.16

0.22

2.11

1.25

1.12

3.11 12 
Mead, W. C. Miley, G. H. Milroy, R. D.

Nebel, R. A, Nebel, R. A. Nellson, G. H.

O'Connell, $R$,

O'Shea

PerkIns, L. J.

Perkins, L. J.

Peter, A. M.

Podulka, W. J.

Post, R. f.

Prager, S, C.

Raman, $R$.

Robertson, $\mathrm{S}, \mathrm{H}$.

Ryutov, D. D.

Ryutov, D. D. Santarius, J. F. Sarff, J.S.

Schaffer, M. J.

Schaeffer, M. J

schauer, M. M.

Schmitt, A.

Schmliz, L.W.

Shumlak, U.

Sleck, P, E.

Slemon, R. E.

Slemon, R. E.

Simakov, A. N.

Slough, J.

Sotnlkov, V. I.

Spong, D. A.

Staillard, 8.W.

Sutter, L.

Taylor, R. J.

Thio, Y, C. F.

Thio, Y. C, F.

Thlo, Y, C. F.

Tynan, G.R.

Wilson, J. R.

Wong, H. V.

Woodruff, $S$.

Wurden, G, A.
Potential for Driving TN Fusion Using Sonlc Cavitation

Overview of Science and lssues far Ion-Injected ICE Fuslon

Measurements in RMF Driven FRCs

Experimental and Theoretical Studies of Electrostatic Confinement IEC

Modular Systems as a Route to Economical Fusion Power

Engineering Design of the National Compact Stellarator

Stellarator

Experiment (NCSX)

Fast Particle Confinement Studies on MST using HXR flux and Fokker- RFP

Planck Modelling

Experimental Studies of Space-Charge Dominated Beams for HIF

Heavy lon Fusion (HIF)

Inertial Fusion Energy with the Advanced Fusion Fuels

The Potentlal of Antiprotons as Drivers for Inertial Confinement

Doppler Effect Measurement of Ion Spin-up in an FRC with RMF

Current Drive

Dynamics of strong proton rings in the Cornell Ion Ring Experiment FRC

The Kinetic Stabilizer/Tandem Mirror: Some Issues and

Progress in Energy Confinement in the Reversed Field PInch RFP

Coaxial Helicity Injection for Plasma Start-Up in NSTX

Application of the Penning trap to the study of neoclassical

Active experiments on determining the quality of magnetlc flux

surfaces in a spheromak

Drift Instabslities in a strongly collisional, beto $>1$ plasma

Prolfferation-Proof Fusion Power

Confinement Improvement and Future Plans in MST

Advances in NOVEL FRC. Plasmas

Stead-State, Oblate, D-3He FRC Fusion for Terrestrlal Power and

Space Propulsion

Ion Resonance Instability in the Virtual Cathode of the Penning

Fuslon Experiment

Status and Prospects for Laser Direct-Drive for Fuslon Energy

Sawtooth and Edge Plasma Behavior during KCRH Heating in the

Electric Tokamak

Evidence of Stabilization in the ZaP Flow Z-Pinch Experiment

Construction of the Helicity Injected Tons with Steady Inductive

Helicity Injectlon (HIT_SI)

An Overview of Progress on MTF Research

MTF Prospects for Low-Cost Energy Development

Stability of Axisymmetric Plasmas in Closed Line Magnetlc Fields

RMF Driven FRC Fusion Reactor

Fast Ignition of Strongly Magnetized Plasma

Alpha Physlcs issues in Compact Stellarators

Considerations of Helicity Balance in SSPX

Reexamining the possibjlity of laser based ifE using a $\sim$ Imicron

ET Results and Burning Plasma Options

Magnetized Target Fusion Driven by Plamsa Liners

Plasma Liner Research for MIF at NASA Marshall Space Fllght

Conceptual Design of an MTF Space Propulsion System

DISCUS: A very high beta dipole disk-like equilibrium physics

experimental concept

Physlcs Progress on the NSTX Device

Hlgh beta stability of magnetic dipole configurations

Heuristic model to explain the slow formation of spheromaks in

SSPX without a globaliy coherent $n=1$ mode

Progress on the FRC-L FRC Plasma Injector at LANL for Magnetized MTF

Target Fusion
Mlsc.

IEC

FRC

Poster 3

3.13

Oral 4

0.41

Poster 1

1.17

Oral 4

0.42

Skunkworks

Poster 2

2.08

Poster 1

Poster 1

1.27

Poster 1

1.28

Skunkworks

Poster 1

1.18

Poster I

Skunkworks

Oral 1

Poster 2

0.11

2.03

2.20

1.07

Spheromak

Poster 1

Skunkworks

RFP

FRC

FRC

Poster 3

1.01

Poster 1

1.22

Poster 1

Poster 2

Oral 2

0.21

Poster 2

Flow Z-PInch

Oral 5

0.52

Poster 1

Oral 5

0.51

Poster 3

3.01

Poster 2

Poster 1

Poster 1

Poster 2

Poster 1

2.17

1.19

1,30

2.07

1.04

Oral 3

0.32

Poster 3

3.08

3.09

3.10

2.19

0.31

Oral 3

2.18

IDX

Poster 2

1.05

Poster 3

3.02 


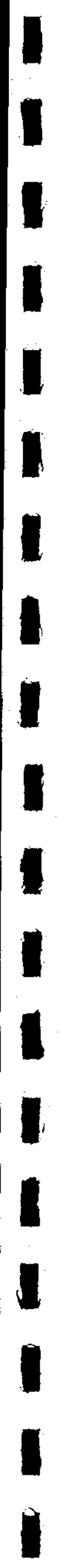

Oral Sessions 


\title{
The Maryland Centrifugal Experiment : Motivation and Status
}

\author{
R.F. Ellis, D.K. Gupta, A.B. Hassam, S. Messer, University of \\ Maryland
}

The Maryland Centrifugal Experiment (MCX) : Motivation and Status, R.F. Ellis, D. K.Gupta, A. B. Hassam, S. Messer, University of Maryland. The concept of centrifugal confinement is to employ centrifugal forces from supersonic rotation to enhance magnetic confinement along magnetic field lines allowing an open magnetic configuration. Rotation is driven by an applied electric field which is perpendicular to the confining magnetic field (ExB rotation). The plasma rotation will be nonuniform resulting in large velocity shear which should stabilize MHD instabilities and possibly quell microturbulence, leading to near classical confinement. MCX is designed to test these ideas. The geometry of the magnetic field is that of a solenoid with axisymmetric mirror end fields. Biasing of an inner core relative to the outer wall produces a radial electric field which will drive supersonic azimuthal rotation and the resulting centrifugal force will contain plasma to the solenoidal portion. Simple theoretical calculations as well as detailed 3D MHD+transport modeling (Numerical Maryland Centrifugal Experiment) will be presented, all of which support the concept. The design principles and status of MCX, which is in the construction phase, will be summarized, with details presented during the poster session. In brief, MCX is approximately $4 \mathrm{~m}$ in length with central diameter of $0.6 \mathrm{~m}$ with $\mathrm{B}$ in the $0.2-2 \mathrm{~T}$ range; expected parameters are: plasma density $1-10 \mathrm{x}$ $10^{13} \mathrm{~cm}-3$, temperature 10-50 eV, Mach number greater than 2. 


\title{
Progress in Levitated Dipole Research
}

\author{
D. T. Garnier, A. K. Hansen, M. E. Manel, T. Sunn Pedersen, E. Ortiz \\ Columbia Iniversity, Dcpartmenl of Applied Physics and Applied Mathematics \\ J. Kesner, C. M. Jones, I. Karim, J. Minervini, P. Michael, \\ A. Radovinsky, J. Schuitz, J3. Smith, A. Zhukovsky \\ Massachusetts Institute of Technology, Plasma Science and Fusion Center
}

The levitated dipole is a unique plasma confinement concept. Originally suggested by Hasegawa based on his study of magnetospheric plasmas, it features an internal levitated circular magnet surrounded by a large vacuum chamber. The magnetic geometry is approximated by a simple magnetic dipole, and has closed field lines. Without rotational transform, the plasma equilibrium is stabilized by plasma compressibility rather than by average good curvature. The marginal condition for stability requires that the plasma pressure profile be stationary under an adiabatic interchange of flux tubes; i.e. the entropy $s \equiv p^{\gamma \gamma}=$ constant, where $V \equiv \oint d \ell / B, \gamma=5 / 3$. Another consequence of closed field line geometry is that the plasma potential is not necessarily a flux function, leading to the possibility of convective flows. While convective cells are often associated with an increase in thermal transport, convective cells move plasma particles without convecting energy in plasmas with constant entropy profiles because of the adiabatic condition. Understanding the behavior of plasmas stabilized by compressibility, the formation of convective cells, and the resultant transport properties are the major challenges of levitated dipole research.

Recent study of linear drift wave stability in dipoles have included the effect of varying plasma temperature and density profiles. Drift wave stability exists for profiles with constant $s$ and $\eta \equiv$ $d \ln T / d \ln n>2 / 3$. Other groups have explored the non-linear evolution of closed field line systems showing the formation of convective cells for profiles above and near marginal stability. Results from recent simulations show the formation of convective cells and non-local transport which evolve the plasma towards constant entropy and stationary density and temperature profiles.

The Levitated Dipole Experiment (I,DX), a team effort of Columbia University and MIT, is a world-class superconducting experiment designed to illuminate the physics of dipole confinement. LDX, currently under construction and nearing first operations, consists of a $1 / 2$ ton, $1.5 \mathrm{MA}, 5 \mathrm{~T}$ magnet levitated within a $5 \mathrm{~m}$ diameter vacuum vessel. The floating coil ( $F$-coil), with its advanced $\mathrm{Nb}_{3} \mathrm{Sn}$ superconductor and statc-of-the-art cryostat will be cooled by a cryogenic heat exchanger and then charged inductively by a 1:3 M.J NbTi superconducting charging-coil. After launching by a mechanical launcher/catcher dovice. the F-coil will be magnetically supported by a high- $T_{c}$ superconducting levitation-coil. F-coil motion will be diagnosed with an 8 laser optical system and levitation will be continuously feedback stabilized by a digital control computer over the 8 hour run day. Work on all these systems, as well as plasma heating and diagnostic systems, is well advanced and the status of their construction will be presented.

First plasma experiments will focus on the stability of high- $\beta$ dipole plasmas produced by ECRH, with the F-coil initially in a supported mode. In later experiments the coil will be levitated to eliminate mirror losses and allow for higher plasma ${ }^{3}$. 'The presentation will conclude with a discussion of the experimental plan and the associated base diagnostic set for high- $\beta$ LDX operations. 
Overview of Recent Results from the SSPX Spheromak Experiment

D.N. Hill for the SSPX Group

Lawrence Livermore National Laboratory, Livermore, CA 94550 USA

The Sustained Spheromak Physics eXperiment is now in its third year of operation. In the past year we have added a new set of bias magnetic field coils to increase shaping flexibility and installed a new inductor which has allowed us to extend the pulse length and increase energy throughput by a factor of three. We are exploring the physics of driven spheromaks, specifically the relationship between injector current, electron temperature, and the resulting strength of the confining magnetic fields.

The SSPX device produces 500-600kA spheromak plasmas with edge magnetic fields of $0.25 \mathrm{~T}$, densities in the range of $0.5-2.0 \times 20 \mathrm{~m}^{-3}$, and peak electron temperatures of $150 \mathrm{eV}$ while driven. The plasma is sustained by coaxial DC helicity injection. With the new bias magnetic field coils, we have lowered the threshold injector current density $\left(\lambda=\mu_{0} \mathrm{j} / \mathrm{B}\right)$ from about $\lambda=20 \mathrm{~m}^{-1}$ to around $10 \mathrm{~m}^{-1}$, which about equal to the flux conserver eigenvalue, $\lambda=\left(3.83^{2} / \mathrm{R}^{2}+\pi^{2} / \mathrm{H}^{2}\right)^{1 / 2}$, where $\mathrm{R}$ is the flux conserver radius and $\mathrm{H}$ its height. This makes it possible to drive the spheromak with edge current densities more nearly equal to those corresponding to the fully-relaxed, force-free Taylor state with constant $\lambda$ throughout the plasma volume. Lower fluctuation levels and peaked temperature profiles are then observed. The nT product follows what appears to be a beta limit corresponding to a peak $\beta_{\mathrm{e}} \approx 6 \%$ and a volume average $\beta_{\mathrm{e}} \approx 4 \%$. Ion temperatures inferred from Doppler-broadening of impurity emission lines show $\mathrm{T}_{\mathrm{i}} \sim$ $\mathrm{T}_{\mathrm{e}}$, yielding a peak total $\beta \sim 12 \%$. The existence of this $\beta$ limit suggests that higher performance might follow if we can produce higher field spheromaks. This, in turn, points to increasing the current amplification (presently $\mathrm{I}_{\text {tor }} / \mathrm{I}_{\text {gun }} \sim 2.0$ ) to better utilize the existing capacitor bank.

Most recently, we have begun exploring very slow formation and buildup using an external inductor to extend the current pulse from 1 to $3 \mathrm{~ms}$. The inductor limits the rise time of the current to about $0.4 \mathrm{~ms}$ instead of $150 \mu \mathrm{s}$. In these discharges we observe steadily increasing field energy in the spheromak at constant injector current. Current amplification is improved by more than $50 \%$ and continues to increase until the capacitor bank driving the spheromak runs out of energy. The continual rise of current and field in the spheromak is observed both with and without the presence of large amplitude $n=1$ fluctuations. Helicity balance measurements point to the importance of determining both the captured magnetic flux and the sheath voltage at the injector electrodes, which has until now been neglected. Power balance shows that most of the input energy is ultimately conducted to the divertor plate and is not dissipated by radiation or charge exchange losses. The role of the magnetic geometry and plasma temperature in controlling the buildup rate are now being explored.

This work was performed under the auspices of US DOE by the University of California Lawrence Livermore National Laboratory under Contract No. W-7405-ENG-48. 
Field Reversed Configurations -- Issues, Status, and Plans Alan Hoffman, University of Washington

FRCs are an attractive reactor confinement scheme due to their high beta, simply connected geometry, and natural divertor. The principal issue is whether such a simple geometry, with no toroidal field, can be stable and have adequate confinement. The favorable stability behavior seen in present experiments has been attributed to kinetic effects represented by a small ratio, $s$, of the number of internal ion gyroradii between the field null and the separatrix. Stable FRCs with s values up to 4 were produced in the Large s Experiment (LSX). No theory has yet been successful in explaining this stability, but progress is being made based on particular elongated profiles and possible minimum energy states involving conservation of generalized helicity. More immediate problems are to develop technologically attractive formation and improved lifetime. High voltage theta pinches, trapping an initial reverse bias field, have been the standard device for forming hot FRCs, and recently the technique of merging more slowly formed spheromaks with opposite helicity has been developed, but both methods are probably limited to forming FRCs with only tens of $\mathrm{mWb}$ fluxes and sub-msec lifetimes, insufficient to study the high $\mathrm{s}$ regime required for reactor relevance. Recently methods of creating, building up the flux, and sustaining FRCs using particle beams and rotating magnetic fields (RMF) have begun to be explored. Present FRCs have too little flux to adequately trap high energy ions, but Neutral Beam (NB) injection experiments in Japan have demonstrated improved confinement in FRCs with a mainly axially directed (confined by end mirrors) small high energy ion component. RMF experiments in the US, based on the Australian developed rotamak technique, have also recently demonstrated formation and quasi-steady sustainment of standard, flux confined, prolate FRCs, but only at low density and temperature due to insufficient power to overcome initial radiation barriers, with a resultant high plasma resistivity. These RMF sustained FRCs are interesting in having a very low separatrix density due to a strong inward particle drive. Experiments are underway to try to build up and sustain the flux of hot FRCs formed in the normal theta pinch manner and then translated and expanded into a chamber containing RMF antennas. FRCs formed, translated, and then trapped between mirrors in such a manner have been noted in both US and Japanese experiments to have lower resistivity than predicted by previous high density derived empirical scaling $\left(D_{\perp} \sim\right.$ $10 / \mathrm{n}^{1 / 2}\left(10^{20} \mathrm{~m}^{3}\right) \mathrm{m}^{2} / \mathrm{s}$ ), which should provide a good test of the RMF current drive scheme. If such experiments are successful, the eventual plan would be to add low energy neutral beams to both counteract the RMF produced torque, assist in current drive, and hopefully favorably affect the plasma resistivity. 


\section{Fast Ignition}

\section{H Key, LLNL}

Fast ignition has significant potential advantages for IFE relative to conventional hot spot ignition, notably increased gain and reduced requirements for fuel density and compression symmetry.

It relies however on very new and complex physics for laser generation of intense relativistic electron beams or multi- $\mathrm{MeV}$ proton beams and the transport of these beams to the ignition hot spot.Validation of the feasibility of ignition by either electrons or protons is a key goal of current research .

The current status of fast ignition will be reviewed and illustrated by results from a recent OFES funded concept exploration project focused on the energy transport problem. The project is a LLNL/GA/UC Davis/Princeton collaboration and experiments have been carried out mainly through international collaborations in Europe. 


\title{
Innovation in the Stellarator Concept: An overview of new US stellarator experiments.
}

\author{
S. Knowlton \\ Department of Physics \\ Auburn University
}

As an alternate approach to fusion research, the stellarator is a mature concept. Nonetheless, relatively recent advances in theory and computation have led to the development of innovative stellarator concepts in which the magnetic configuration exhibits a near-symmetry in a specific direction. Among the potential benefits of introducing quasi-symmetry to the stellarator concept are greatly improved neoclassical confinement in the low collisionality regime as well as reduced neoclassical flow damping. If proven successful, the application of quasi-symmetry to helical devices could combine the good confinement of the axisymmetric tokamak with the inherent steady-state, low disruptivity operation of the stellarator for overall improved toroidal plasma performance. Furthermore, practical stellarator configurations with lower aspect ratio and higher beta limits than conventional designs have been developed through extensive optimization procedures.

New stellarator experiments in the US will test these theoretical advances: the operating HSX device is investigating neoclassical confinement improvement in a quasihelically symmetric plasma, the planned proof-of-principle scale NCSX experiment will address integrated issues of disruption-free, high-beta operation in a quasi-axisymmetric configuration with high input power, and the proposed QPS experiment will examine confinement and stability of quasi-poloidally symmetric plasmas in a very low aspect ratio device. While predicted bootstrap currents in finite-beta realizations of all of these configurations are lower than in advanced tokamaks, low aspect ratio stellarators will operate with moderate levels of plasma current. Therefore, the understanding and avoidance of kink instabilities in helical devices is also a component of this innovative program. The small CTH experiment under construction will investigate current-driven instabilities and the potential for passive suppression of disruptions in helical configurations. 
Innovative Confinement Concepts, January 22-24, 2002, College Park, Maryland "Heavy ion beam science and innovative technology towards inertial fusion energy""

B. Grant Logan, Director, Heavy-Ion Fusion Virtual National Laboratory

Abstract

Demonstration of inertial fusion ignition and energy gain in the laboratory is expected with the National Ignition Facility (NIF) around 2010, which will provide the scientific basis for inertial fusion energy (IFE). In addition, efficient and affordable drivers, mass-produced high gain targets, and long life, low activation chambers for $5-10 \mathrm{~Hz}$ pulse rates, must also be developed for practical IFE. The DOE Office of Fusion Energy Science (OFES) is supporting $\sim \$ 10 \mathrm{M} / \mathrm{yr}$ for heavy-ion fusion beam driver science, $\sim \$ 4 \mathrm{M} / \mathrm{yr}$ for related $\mathrm{HIF}$ chamber and target development, and another $\sim \$ 3 \mathrm{M} / \mathrm{yr}$ for laser IFE (while DOE-DP is supporting about $\$ 25 \mathrm{M} / \mathrm{yr}$ additional for $\mathrm{KrF}$ and solid-state (DPSSL) IFE). HIF is carried out by a virtual national lab (HIF-VNL) with LBNL, LLNL, and PPPL, with other HIF related research carried out by UMd, NRL, MIT, MRC, and UM. HIF related chamber and target development is supported in the Virtual Lab for Technology (VLT) by LLNL, GA, SNL, UCB, UCD and UCLA.

HIF is motivated because high-energy accelerators of MJ-beam energy are consistent with the efficiency, pulse-rate, and longevity required for IFE, and because clear-bore magnets used to focus heavy-ions might be tolerant of target debris and radiation. Transporting and focusing higher space charge beams at lower 10-30 MeV/amu heavy ions required for IFE is the main new challenge. Particle-in-cell simulations are in good agreement with past low current ( $\mathrm{mA}$ level) beam experiments in transport, merging, acceleration and final focus, in which the dimensionless beam perveances (space-charge potential/ion kinetic energy) were driver-like. Higher current experiments are beginning (100 mA to $1 \mathrm{~A}$ ) in high brightness merging beamlet sources, transport at $\sim 2 \mathrm{MeV}$, and ballistic focusing with plasma neutralization. Improved simulation models enable modeling these experiments with realistic (not just idealized) beam distributions. The higher beam space-charge potentials attendant to these higher current experiments will allow us to study the effects of ionization of the residual gas by the beam, the trapping in the beam of stray electrons, and the dynamical effects on the beam of these two processes. A key strategic goal of the program is an integrated and detailed source-to-target simulation capability.

After these separate experiments are completed, the next step will be to study transverse and longitudinal emittance growth through injection, acceleration, longitudinal beam compression, and final focus in an integrated beam experiment (IBX-a candidate proof-ofprinciple experiment to follow NCSX). The integrated beam experiment will be much more challenging because prediction of the final focus spot size with integrated models depends on the accumulated beam phase space changes through each region along the beam system.

HIF has significant potential for lower cost experiments through innovative technology: higher current density merging-beamlet injectors, high field, compact superconducting quadrupoles, and agile-waveform, high gradient injection and induction modules. Experiments on neutralizing beam space charge in the chamber may lead to lower voltage/cost drivers. Finally, innovative HIF target designs may increase allowed beam spot size, and fast ignition may reduce driver energy and lengthen pulse durations as well as allow larger focal spot size.

*This work was performed under anspices of the U.S. Department of Energy by the University of California, Lawrence Berkeley and Lawrence Livermore National Laboratories under Contract Numbers DE-AC0376SF00098 and W-7405-Engr-48, and by the Princeton Plasma Physics Laboratory under Contract Number DEAC02-76CH03073. 
Pulsed Power ICF and IFE Research on Z and ZR*

\section{Keith Matzen** \\ Sandia National Laboratories, Albuquerque, New Mexico USA}

Since its inception as a pulsed-power driver for z-pinch implosions in 1996, $Z$ has become a premiere facility for high energy density physics research relevant to both ICF and IFE. On Z, the high magnetic field pressures associated with 20 -MA load currents implode a wire array zpinch, generating up to $1.8 \mathrm{MJ}$ of x-rays at powers as high as $230 \mathrm{TW}$. These intense $\mathrm{x}$-ray sources have renewed interest in utilizing fast pulsed-power generators to drive ICF capsules. Target design calculations for two specific target configurations (z-pinch-driven hohlraum and dynamic hohlraum) indicate that fusion yields in excess of $500 \mathrm{MJ}$ can be obtained in configurations where the z-pinch implosions are driven by peak currents of $60 \mathrm{MA}$. For the zpinch-driven hohlraum concept, recent experiments on $Z$ with double-pinch hohlraums have demonstrated hohlraum energetics and coupling efficiencies that scale to high yield, and initial radiation symmetry and capsule implosion experiments have also been performed. For the dynamic hohlraum configuration, shock and capsule implosion dynamics have been studied. In addition, we have begun using the Z-Beamlet laser (ZBL) to backlight capsule implosions, and have proposed to study fast igniter physics by adding a PW capability to the ZBL laser. For IFE, recent developments have led to a conceptual approach for a rep-rated z-pinch power plant that exploits the advantages of going to high yield ( $\sim$ few GJ) at low rep-rate $(\sim 0.1 \mathrm{~Hz})$. This concept uses a recyclable transmission line to provide the necessary standoff between the fusion target and the power plant chamber. Based on this progress for both ICF and IFE, and the demand for experiments on $Z$, we have proposed to reburbish $Z$ to increase the shot rate, increase its precision, and improve the current pulse shaping capability and load coupling. Refurbished $Z$ (ZR) could produce over $2.5 \mathrm{MJ}$ and $350 \mathrm{TW}$ of $\mathrm{x}$-rays for ICF and radiation physics experiments.

In this presentation we will summarize recent experimental data supporting high-yield ICF driven by an intense z-pinch-driven x-ray source. We will also describe the conceptual approach to the rep-rated z-pinch power plant for IFE and describe the initial experiments to test the validity of the recyclable transmission line concept.

*Sandia is a multiprogram laboratory operated by Sandia Corporation, a Lockheed Martin Company, for the United States Department of Energy under Contract DE-AC04-94AL85000.

** In collaboration with D. D. Bloomquist, G. A. Chandler, M. E. Cuneo, C. Deeney, M. P. Desjarlais, G. L. Donovan, D. L. Hanson, R. J. Leeper, T. A. Mehlhorn, T. J. Nash, C. L. Olson, J. L. Porter, J. P. Quintenz, G. E. Rochau, L. E. Ruggles, C. L. Ruiz, T. W. L. Sanford, J. F. Seamen, S. A. Slutz, W. A. Stygar, R. A. Vesey, D. F. Wenger, J. H. Hammer, J. D. Lindl, M. Tabak, S. C. Wilks, R. L. Bowers, D. L. Peterson, P. F. Peterson, J. S. DeGroot, and R. R. Peterson 


\section{Overview of Research and Issues for Ion-Injected IEC Fusion}

George H. Miley

University of Illinois, Urbana-Champaign, LENR Laboratory, 103 South Goodwin Avenue, Urbana, IL 61801-2984, USA, g-miley@uiuc.edu

There are two fundamental approaches to IEC fusion, ion-injected and electron-injected devices. In either case, the injected species creates a potential well, which in turn traps the second species; the ultimate goal being the formation of a "double well". Typically, gridded IEC devices are currently employed for ion injection experiments, although in theory the grids can be removed from future power producing devices. Such experiments are presently under study at various labs including the University of Illinois, the University of Wisconsin, LANL, Kyoto University, and University of Sydney. Electron-injected devices include the Penning Trap, Oscillating Plasma IEC, and the Polywell Hybrid Magnetic IEC. Work on electron devices is primarily being done at LANL and EMC2. A brief summary of various experimental results from the ion-injected devices will be presented along with comments about the relationship between it and electron injection.

Several fundamental physics issues remain to be resolved for ioninjected devices. Experimental and theoretical studies verify double well formation, but the volume of the ion trap created is too small in relation to the total device size for a practical fusion unit. Increased ion angular momentum has been proposed as a solution, but this remains to be verified. Stability under high injection current conditions also remains as an issue for scale up to practical devices, although preliminary studies suggest a stability "window" exists. Finally, methods to create and inject ions while maintaining a very low neutral background density remain as a critical issue. Along this line, results from recent RF ion gun injection experiments will be presented. 


\title{
Experimental and Theoretical Studies of Electrostatic Confinement
}

\author{
R.A. Nebel, C.P. Munson, Los Alamos National Laboratory
}

Recent theoretical work $[1,2]$ has suggested that a tiny oscillating ion cloud may undergo a self-similar collapse that can result in the periodic and simultaneous attainment of ultra-high densities and temperatures. Theoretical projections[1] indicate that such a system may have net fusion gain even for an advanced fuel such as D-D. Schemes have also been suggested where a massively modular system consisting of tens of thousands of these spheres can lead to a very high mass power density device (comparable to a LWR),[1] Such systems should be very economically competitive.

However, a major uncertainty in this plasma system is the behavior of the electrons. Previous theoretical work has shown that the required electron cloud is susceptible to an instability which is analogous to the Rayleigh-Taylor mode present in fluid mechanics.[3] In the kinetic limit (counter-streaming electrons) one expects this instability to go over to a type of two-stream instability. Simple two-stream analysis suggests that the mode may be absolutely stable at a finite value of ratio of the Debye length to plasma radius. This conclusion is consistent with previous experimental results. [4]

These experiments are presently being redone on the INS-e IEC. Results indicate fluctuations in the electron plasma frequency $(\sim 60 \mathrm{MHz})$ range that show bursting behavior. The plasma is also showing coherent fluctuations at lower frequencies $(\sim 100 \mathrm{kHz})$. This data will be presented along with plans for this years experimental campaign.

Electron dynamics are also being studied in the Penning Fusion eXperimentIons (PFX-I). A virtual cathode is produced in this device by a nonthermal electron plasma confined in a modified-geometry Penning trap. Ions are then confined in the virtual cathode. RF signals resulting from instability caused by the trapped ions serve as a nondestructive diagnostic of the density of the electron plasma at the virtual cathode. Trapped ions can also be destructively detected by a negatively-biased microchannel plate located below the trap by dumping the electrons out the top of trap, thereby destroying the virtual cathode and releasing the ions.

1. R. A. Nebel, D. C. Barnes, Fusion Technology 38, 28 (1998).

2. D. C. Barnes, R. A. Nebel, Physics of Plasmas 5, 2498 (1998).

3. R. A. Nebel, J. M. Finn, Physics of Plasmas 8, 1505 (2001).

4. R. L. Hirsch, Physics of Fluids 11, 2486 (1968). 


\title{
Advances in Energy Confinement in the Reversed Field Pinch
}

\author{
S.C Prager and the MST Group \\ The University of Wisconsin - Madison \\ Madison, Wisconsin 53706
}

The primary approach to improve confinement in the reversed field pinch (RFP) is to modify the current density profile so as to reduce magnetic fluctuations. To this end, we program the time dependence of the Ohmic loop voltages. Past confinement gains were accomplished with poloidal loop voltage programming. Recently, we have added toroidal loop voltage programming. The goal is to increase the parallel electric field in the outer region of the plasma - to flatten the driven current density profile. The result is that confinement has improved further; the energy confinement time is now about $10 \mathrm{~ms}$ (a ten-fold improvement over the standard RFP), corresponding to thermal diffusivity of about $5 \mathrm{~m}^{2} / \mathrm{s}$. These values exceed the empirical confinement scaling that has characterized all past RFP results, and might be sufficiently low that the effect of electrostatic fluctuations may no longer be negligible. Beta values of $15 \%$ and electron temperature of $1.3 \mathrm{keV}$ are achieved. For the first time in MST, runaway electrons are confined up to $100 \mathrm{keV}$ (measured by hard xray emission). Fokker-Planck modeling suggests that the confinement time of the energetic electrons is about equal to that of the bulk. This implies that the magnetic surfaces are changing from stochastic to wellformed. Ion temperature (measured by charge exchange recombination spectroscopy and Rutherford scattering using diagnostic neutral beams) is unchanged in improved confinement plasmas.

The current density profile is measured with laser Faraday rotation (a UCLA collaboration), and aided by motional Stark effect diagnosis. Interestingly, the current density profile peaks in improved confinement plasmas -perhaps from reduction of the current-flattening effect of the fluctuations (the dynamo effect).

We have gathered new evidence that improved confinement is accompanied by reduced magnetic fluctuations. Faraday rotation measurements yield the first observation of the core magnetic fluctuations, which are observed to reduce from standard to improved confinement discharges by at least a factor of two. First measurement of core particle transport by electrostatic fluctuations in the RFP is obtained from a heavy ion beam probe (an RPI collaboration). Electrostatic transport is small in standard plasmas. Its value in improved confinement plasmas is not yet determined.

Recently, we have investigated plasma behavior in non-reversed plasmas, with the toroidal magnetic field zero, or slightly positive, at the wall. Energy confinement in these plasmas is about equal to that of RFP plasmas. Hence, reversal is not necessary for good confinement. This result is not in conflict with stability or relaxation theory. However, we do observe that anomalous ion heating and anomalous angular momentum transport are absent or weak in non-reversed plasmas. In standard plasmas, these anomalous processes occur during sawtooth crashes. Plasma relaxation is weaker in these plasmas, for reasons not yet discovered.

Future plans include RF wave current drive, oscillating field current drive (ac helicity injection) and neutral beam injection for further plasma control. 


\title{
Status and prospects for laser direct-drive for fusion energy
}

\author{
Andrew J. Schmitt \\ Laser Plasma Branch \\ Plasma Physics Division \\ Naval Research Lab
}

Direct drive laser fusion has been an innovative fusion concept for the past twenty five years. Its utility for energy is dependent upon the possibility of achieving high energy gains, which puts stringent constraints on the uniformity of the laser and target. Within the past twenty or so years, the implementation of smaller wavelength lasers and discovery of optical smoothing methods have all but eliminated laser-plasma coupling issues for direct-drive and placed us closer to satisfying the symmetry constraints. More recently, the increased use of large two (and sometimes three dimensional) radiation-hydrocodes have enabled more quantitative assessment of the performance and stability of high-gain target designs. Recent experimental results also show the promise of reducing laser imprint further. We will present these and other results and discuss the status and prospects for high gain using the direct drive laser concept. 


\title{
Evidence of Stabilization in the ZaP Flow Z-Pinch Experiment
}

\author{
U. Shumlak, B. A. Nelson, J. E. Bright, E. A. Crawford, R. P. Golingo, \\ S. L. Jackson, \\ Aerospace \& Energetics Research Program, University of Washington \\ D. J. Den Hartog, \\ University of Wisconsin
}

The stabilizing effect of an axial flow on the $m=1$ kink instability in z-pinches has been studied numerically by reducing the linearized ideal MHD equations to a one-dimensional eigenvalue equation for the radial displacement. The principal result reveals that a sheared axial flow stabilizes the kink mode when the shear exceeds a threshold value which is inversely proportional to the wavelength of the mode. Nonlinear simulations support the stabilizing effect by showing the stabilization of the $m=0$ sausage mode when the equilibrium contains a sheared axial flow.

The ZaP Flow Z-Pinch Project at the University of Washington seeks to experimentally determine the connection between stability and plasma flow by generating Z-pinch plasmas with an inherent axial flow. The experiment produces Z-pinch plasmas $50 \mathrm{~cm}$ in length by initiating the plasma with a one meter coaxial gun. The coaxial gun generates the axial plasma flow. After leaving the coaxial gun the plasma assembles along the axis to form a flow Z-pinch. Magnetic probes measure the acceleration and assembly process, as well as. the evolution of fluctuation levels of the azimuthal modes $m=0,1,2$. Axial flow profiles are determined by measurements of the Doppler shifts of impurity lines. Experimental results show a stable period which is over 700 times the expected instability growth time in a static Z-pinch. The experimentally measured axial velocity shear is greater than the theoretical threshold during the stable period and approximately zero afterwards when the magnetic mode fluctuations are high. Time-dependent density measurements are made using a laser interferometer. Gross plasma motion is determined by using a fast framing camera to detect visible emission. An overview of the experimental program and results will be presented. 


\section{An overview of progress on MTF research}

R.E.Siemon, D.C.Barnes, P.N.Assmus, W.J.Feinup, T.P.Intrator, R.C.Kirkpatrick, C.E.Knapp, I.R.Lindemuth, M.C.Langner, R.J.Maqueda, K.F.Schoenberg, K.J.Scott, P.T.Sheehey, E.M.Tejero, J.M.Taccetti, M.G.Tuszewski, Z.Wang, G.A.Wurden, S. Zhang,

Los Alamos National Laboratory, Los Alamos, NM 87545

J.H.Degnan, C.Grabowski, S.K.Coffey, M.H.Frese, D.Gale, E.L.Ruden, G.J.Marklin, W.Sommars, P.J.Turchi

Air Force Research Laboratory, Albuquerque, NM 87117

P.Parks, M.J.Schaffer

General Atomics, San Diego, CA 92121

D.D.Ryutov

Lawrence Livermore National Laboratory, Livermore, CA 94550

Y.C.F.Thio, J. Cassibry, R.Eskridge, M.Lee, A. Martin, G.R.Schmidt, J.Smith, S.T.Wu NASA Marshall Space Flight Center and Univ. of Alabama, Huntsville, AL 35812

\section{B. Bauer, V. Makhin, R. Presura \\ University of Nevada, Reno}

Magnetized Target Fusion (MTF) has emerged in recent years as a qualitatively different approach or "pathway" to fusion energy. The major MTF issues have been identified, and an MTF Research Coordinating Committee (underlined author names) has been formed to facilitate communication between the institutions involved. Posters will report on the following issues:

1. Target formation with adequate temperature, lifetime, and density

- At Los Alamos the new FRC target generator called FRX-L is beginning to operate with the needed electrical parameters to form a suitable MTF target

- New theoretical results from Los Alamos appear to explain the long-standing puzzle of FRC stability for the case of a highly prolate geometry.

- At GA wall-confined oblate FRC targets have been studied that might allow MTF target formation at high $-\mathrm{s}$ while avoiding ideal MHD tilt and interchange instability.

- LLNL studies show that drift instabilities in the MTF, $\beta>1$, strongly-collisional regime are generally weaker than in previously studied collisionless regimes.

- At AFRL the MACH2 code has been modified to allow study of FRC formation

- Calculations suggest that the basic physics of a wall-confined target plasma could be studied using the Nevada Terawatt Facility.

2. Liner heating (wall compression) without mixing impurities

- AFRL has found reasonable efficiency with a theta-pinch-coil driven liner, which avoids z-pinch tapered electrodes and is more suitable for target injection.

- 3-D hydro calculations show that plasma jet liners can be formed, and the implied energy requirements, implosion trajectories, and fusion gain appear suitable to NASA.

3. The application of MTF technology to practical energy or space propulsion.

- A simple generic argument for MTF shows semi-quantitatively why MTF is less expensive than conventional fusion approaches.

- In system studies for space propulsion, MTF appears to deliver the required specific power and specific impulse with relatively low system mass at LEO (Low Earth Orbit). 


\section{ET Results and Burning Plasma Options.* \\ R.J. Taylor, University of California, Los Angeles (UCLA), CA 90024}

Central plasma confinement times close to 1 second are routinely obtained in the Electric Tokamak at $1 / 4$ Tesla fields. Consequently, the energy confinement in ET has exceeded our predictions by about a factor 5. Some of this improvement is related to higher-than-expected densities and some of it is due to an intrinsic improvement in the neo-ALCATOR scaling coefficient $\tau_{\mathrm{E}}(0) \approx 0.3 \mathrm{M}(0) \mathrm{RaB}^{2 / 3}$ (MKS), where $\mathrm{M}$ is the Murakami number. The plasma pulse length has been extended to 3 seconds and we expect trivial extension to 10 seconds soon. No impurity problems exist with ICRF heating up to the $300 \mathrm{~kW}$ level applied on two antennas. More antennas are now ready but RF interference to the control systems has limited full utilization of the available power. The exceptional confinement results, at 1/4 Tesla fields, extrapolate well to D-T and advanced fuel burners but not yet to an economic fusion reactor without the near unity beta core. Achieving high betas is our next challenge. Nevertheless, our results up to date are so encouraging, for large size but low field devices, that a preliminary analysis on the ignitability of ET/DT device in first stability and at 2 Tesla fields will be presented and then extended to the unity beta requirements for economic devices.

*This Research Supported by US DOE grant DE-FG03-86ER53225 


\section{Physics progress on the NSTX Device}

J.R. Wilson, PPPL

The NSTX research program is structured to explore the physics of the Spherical Torus and to establish the potential of this configuration as a fusion device. In the past year significant progress has been made in exploring the physics of the NSTX device particularly in the area of plasma transport. Unusual behavior in the energy balance points to a non-understanding in the relative role of electron and ion energy transport in neutral beam heated discharges. Technical progress has been made both in plasma current initiation via coaxial helicity injection and in creating discharges with a large bootstrap current fraction. 
Poster Sessions 
II

in

|1

1.

1

I

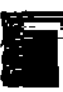

T.

$\bar{F}$

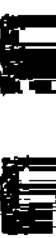

$F$

F 


\section{FRC Stability Explained?}

D.C. Barnes, Los Alamos National Laboratory

More than 3 decades after the first FRC formation, stability remains an enigma. Extensive particle simulations have not yet explained why FRC's are exempt from the internal tilt, and a host of other axial modes (those for which the field lines shift primarily along the axis of symmetry). In spite of this indictment, real world FRC's happily thumb their noses (one per end) at unconvinced theorists and persist for transport times, provided that they are kinetic and long. This paper describes a nearly complete theory of long FRC stability. Special things happen in the long case:

1. Only one pressure profile is allowed

2. Axial modes localize to a single field line

3. There is precisely one unstable mode on each field line (this is independent of toroidal mode number!)

4. These modes are stabilized when the Hall term is considered 5. There is good agreement between the Hall stability condition and Tuszewski's empirical region of good FRC behavior

6. Ion FLR effects will add to the Hall stability but..

7. Some ions resonate with the stabilized modes (their toroidal drift averages to match the stable mode rotation rate) and destabilize them again but..

8. These destabilized modes either are stabilized by modification of the ion distribution function or saturate at very low amplitude.

Point 1 appeared in November 2001 Physics of Plasmas. Points 2-5 are accepted by Physics of Plasmas. Point 6 is trivial. Point 7 was already shown by the results of Barnes, et al. '86 paper and confirmed recently by Belova and others. Point 8 probably explains why kinetic stabilization really does work in long FRC's, and will be discussed in the paper. 


\title{
Energy transport in wall-confined plasmas relevant to Magnetized Target Fusion (MTF)
}

\author{
B.S. Bauer, V. Makhin, R. Presura, S. Fuelling \\ University of Nevada, Reno \\ R.C. Kirkpatrick, I.R. Lindemuth, P. Sheehey, and R.E. Siemon \\ Los Alamos National Laboratory
}

From an overall fusion system perspective, the possibility of compressing a plasma target with beta greater than unity appears very exciting [1]. The confinement of magnetized high-beta plasma directly by material walls has been considered theoretically [2] and computationally [3], but only a little experimentally $[3,4]$. The reason is partly that considerable power input to a plasma is required to establish the density and temperature profiles associated with the transition from thermonuclear temperatures to $\mathrm{eV}$ temperature at the boundary. Plans are being formulated to deploy the 2-terawatt Zebra generator at the Nevada Terawatt Facility (NTF) to perform well-characterized experiments on plasmas with parameters relevant to MTF. The geometry considered is a cylindrical inverse pinch, in which a current pulse on a central conductor drives a plasma current sheet radially outward, until it stagnates and thermalizes when it encounters the inner surface of the outer, conducting, return-current cylinder. A similar inverse-pinch geometry has been studied in the context of plasma opening switches [5]. The plasma is expected to evolve into a stable, magnetostatic equilibrium, with radial plasma pressure gradients. The kink mode is stabilized by the close outer wall. Confinement in the axial direction depends on wall confinement. Thin sheaths next to the conducting end plates contain steep temperature and density gradients perpendicular to the wall (and the azimuthal magnetic field), but no magnetic force or pressure gradient in this (axial) direction. Simple analytic estimates and a well-benchmarked two-dimensional radiationMHD simulation code called MHRDR indicate that a few-hundred-eV deuterium plasma should be formed, with density around $10^{17} \mathrm{~cm}^{-3}$ in the central region. The plasma density, temperature, and impurity content will be measured with EUV spectroscopy and an array of magnetic field ("B-dot") probes. According to MHRDR, the plasma should take microseconds to cool, even in the presence of considerable convection. This cooling rate is much slower than would result if free-streaming losses of ions or unmagnetizedelectron conduction losses were present. Such a slow cooling rate would verify expectations for wall-confined MTF target plasmas. Experimental verification and understanding of the energy transport in this simple wall-confined plasma would provide increased confidence in the design of integrated liner-on-plasma experiments.

1. R.E. Siemon et al., Proc. ITC-12 (2001), and poster this conference.

2. G.E. Vekshtein, Rev. Plasma Physics 15, Consultants Bureau, NY (1990).

3. I.R. Lindemuth et al., Phys. Rev. Letter 75, 1953 (1995).

4. B. Feinberg, Plasma Phys. and Contr. Fusion 18, 265 (1976).

5. B.V. Weber et al., IEEE Trans. On Plasma Science 25, 189 (1997). 


\title{
Fast ignition of liner-compressed fusion fuel
}

\author{
B.S. Bauer \\ University of Nevada, Reno
}

Fast ignition [1] could be an important complement to conventional inertial confinement fusion, offering increased fusion energy gain, decreased implosion drive energy, decreased symmetry requirements, simplified target design, and new perspectives on fusion burn. Compressed DT would be locally ignited by an intense laser, and then, in turn, ignite lower-density $\mathrm{D}_{2}$. Current-driven-liner $(\mathrm{j} x \mathrm{~B})$ compression $[2,3]$ could be an inexpensive method of fuel preparation. In addition, the large magnetic field generated could shrink the region heated by the intense laser, reducing the laser energy required for ignition. Isentropic compression with a programmed voltage pulse would maximize fuel density. Radiative cooling of the liner will help keep the fuel pressure at the Fermidegenerate minimum, $p \sim 0.05 n e^{5 / 3} h 2 / m$. Growth of instabilities in the liner compression could be mitigated by rotational and axial shear flow, resulting in compression of fuel to very high density. Options for creating such flow include making the liner in the form of a corrugated helical sawtooth-shaped capillary tube. Many of the numerical models and facilities needed to explore this scheme are currently available or under development. Fast ignition of liner-compressed fusion fuel appears to be a promising and cost-effective area of fusion science to explore.

1. M. Tabak et al., Phys. Plasmas 1, 1626 (1994).

2. F. Winterberg, Z. Naturforsch. 54a, 459-464 (1999).

3. S.A. Slutz et al., Sandia National Lab Report SAND98-2269 (1999). 


\title{
How initially vacuum flux tubes develop uniform cross-section when an axial current is made to flow
}

\author{
P. M. Bellan, Caltech
}

A new model describing a previously unrecognized fundamental MHD process is presented. This model is inspired by recent solar coronal observations but should also be quite relevant to fusion plasmas. The model explains how initially axially non-uniform, potential-like flux tubes tend towards having axially invariant crosssections via complex bi-directional, flux-conserving plasma flows. It also explains several other enigmatic features of flux tubes which have been observed in the solar corona and which should have lab counterparts, namely: rotating jets emanating from the flux tube ends and traveling axially towards the middle to form counterstreaming beams, ingestion of material from the ends, and elevated pressure/temperature compared to adjacent plasma. The model shows that when a steady current flows along a flux tube with a bulging middle (i.e., a flux tube that is initially produced by a potential magnetic field), non-conservative forces develop which accelerate fluid axially from both ends towards the middle. Remarkably, this axial pumping of fluid into the flux tube causes the flux tube cross-section and volume to decrease in a manner such that the flux tube develops an axial uniform cross-section as observed in coronal loops. The pumping process produces counter-rotating, counter-streaming Alfvenic bulk motion consistent with observations. Collision of the counter-streaming beams at a stagnation point causes bulk heating. Collision of particles bouncing between the approaching beams results in Fermi acceleration of a small fraction of the particles to extremely high energies (i.e., formation of a highly energetic tail). This model has relevance to astrophysical jets and coaxial spheromak guns and explains why these systems tend to form an axial jet along the geometric axis (such as has been observed in observations, experiments, and numerical simulations). 


\title{
Kinetic Stability of the Field-Reversed Configuration ${ }^{1}$
}

\author{
E. V. Belova, R. C. Davidson, H. Ji, and M. Yamada \\ PPPL, Princeton NJ 08543
}

The global stability of the Field-Reversed Configurations (FRC) has been investigated using hybrid (fluid electrons and kinetic ions) simulations. The role of kinetic effects on the $n=1$ tilt mode and other global modes in prolate FRCs have been studied. The linearized hybrid simulations show that there is a significant reduction in the tilt mode growth rate when $\bar{s}<2$, but no absolute linear stability has been found even in the highly kinetic regime when the ion distribution function is taken to be Maxwellian, where $\bar{s}$ is the approximate number of ion gyroradii between the field null and the separatrix. One of the possible explanations of the instability existing beyond the FLR theory stability threshold is the resonant interaction of the wave with ions for which the Doppler shifted wave frequency matches the betatron frequency. The evidence for the existence of betatron resonance-driven instabilities in the large Larmor radius (kinetic) regime is presented. It is shown that the resonant particles contribute significantly to the instability drive and can be responsible for a finite linear growth rate in the large gyroradius regime. The results of nonlinear hybrid simulations at low values of $\bar{s}$ show that the nonlinear evolution of the tilt mode is different from that of the MHD model, and the nonlinear saturation of the tilt instability becomes possible. The saturation occurs due to nonlinear wave-particle interaction, and the nonlinear deviation of the ion distribution function from a local Maxwellian. The effects of realistic boundary conditions and Hall stabilization are also investigated.

\footnotetext{
${ }^{1}$ Research supported by the Department of Energy Contract No. DE-AC02-76-CHO-3073
} 


\section{Numerical Simulations of Compressional Alfven Modes in NSTX*}

E. V. Belova, N. N. Gorelenkov, R. C. Davidson, C. Z. Cheng, and E. D. Fredrickson $P P P L$, Princeton NJ 08543

Hybrid MHD/particle ion simulations are performed to study the excitation of Compressional Alfven Eigenmodes (CAE) by resonant interaction with energetic ions in NSTX. Recent experimental observations and analytic calculations ${ }^{\dagger}$ suggest that $\mathrm{CAE}$ are excited by energetic neutral beam ions during neutral beam injection in NSTX. The modes are predicted to be localized near the plasma edge on the low magnetic field side, and driven unstable through cyclotron resonance with the beam ions. The strong anisotropy in the fast-ion pitch-angle distribution provides the energy source for the CAE instability. In the numerical model, energetic ions are treated using delta-f particle simulations, while the one-fluid resistive MHD description is used to represent the background plasma. The two plasma components are self-consistently coupled using a current coupling scheme. Self-consistent equilibria with anisotropic fast ion pressure has been calculated. It is shown that contribution of the fast ions with energy $E=80 \mathrm{keV}$ and density about $3 \%$ can be comparable to that of the background plasma, and the equilibrium profiles are significantly modified in the presence of the beam ions. Initial simulation results for $\mathrm{CAE}$ will be presented.

*Research supported by U.S. Department of Energy

${ }^{\dagger}$ N.N. Gorelenkov and C.Z. Cheng, Nucl. Fusion 351743 (1995) 


\title{
Sheared magnetofluids and Bernoulli confinement.
}

\author{
Roger D. Bengtson, S. M. Mahajan, H. J. Quevedo, and P. M. Valanju. \\ The University of Texas at Austin.
}

New magnetofluid states that differ qualitatively from those accessible to either neutral fluids or to conventional MHD plasmas have been predicted theoretically. [S. M. Mahajan and Z. Yoshida, Phys. Rev. Lett. 81 4863(1988)] They are predicted to appear if plasmas with strong velocity shear flows with large initial values of both magnetic and magnetofluid helicity are created and allowed to relax. The dynamic invariance of these two helicities will force the plasma to self-organize and to relax to a long lived quasi equilibrium state away from thermal equilibrium. We have built a magnetic mirror device designed to create and investigate these theoretically predicted pressure confining magnetofluid states. Our machine has a central bias rod to create a radial electric field for generating fast plasma flow, a large mirror ratio for good centrifugal confinement, and probes to measure the plasma rotation profiles and fluctuations. Initial results will be presented demonstrating plasma rotation. 
3
0
0

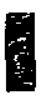

$=0$
$\therefore$
$\vdots$
$\vdots$

$\because:$
$\therefore$

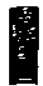

$\because$

$\because$

:

$\therefore$

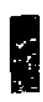

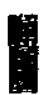

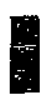

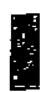

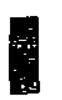

I

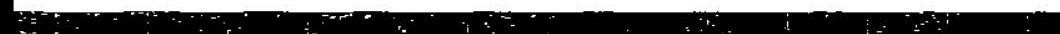




\author{
RMS - Repetitive Merging of Spheromaks: \\ A Steady-State ffusion Reactor with Pulsed Heating, Fueling, and Current Drives* \\ R.F. Bourque, P.B. Parks, and M.J. Schaffer \\ General Atomics, P.O. Box 85608, San Diego, California 92186-5608
}

\begin{abstract}
Despite its nascent stage of development, the spheromak configuration offers a variety of possible formation and operating scenarios, any of which may lead to an attractive fusion reactor power plant. We have carried out a zero-D time-dependent scoping study for a new spheromak concept that uses colliding compact toroids ( $\mathrm{CTs}$ ) injected, at high velocity if needed, along the geometric axis into a main plasma chamber. The repetitive injection of CTs supplies inductive energy and heating in order to gradually build up the main spheromak plasma to full performance steady-state reactor conditions and sustain it there. Plasma poloidal beta remains below the stability limit of $\sim 10 \%$, by virtue of the very high toroidal plasma current $\mathrm{I}_{\mathrm{p}} \sim 85 \mathrm{MA}$ which is obtained during the gradual $\sim 2 \mathrm{~h}$ inductive energy rampup phase that uses fairly modest CT formation hardware. A near by copper shell stabilizes the plasma to fast rotating external modes under the resistive wall condition, and external moderate-field superconducting coils outside the reactor blanket provide the equilibrium magnetic field and shape control.

The reactor scoping study employed several performance assumptions, were chosen to be conservative, such as efficiency and maximum repetitive rate of the RACE-like CT injectors and capacitor banks, and an RFP-like heat transport scaling model in which $\tau_{e} \sim I_{p}$. Ions and electrons are treated separately and models were written for partitioning of thermal, kinetic and magnetic energies. Woltjer Taylor relaxation with helicity conservation is included, as is the dynamo effect that converts toroidal flux to poloidal flux and releases some magnetic energy to heat. This heating mechanism may eliminate the need for high-speed injection. Key physics issues also being addressed are reconnection and coalescence phenomena, and the effect of the merging processes on confinement and stability.

A $500 \mathrm{MW}$ (e) net power RMS plant was studied which had a modest neutron wall loading of $1.9 \mathrm{MW} / \mathrm{m}^{2}$. The recirculating power in steady state was only $\sim 8 \%$. The initial startup power was $\sim 250 \mathrm{MW}(\mathrm{e})$. The startup energy was recovered in only two hours of operation on the grid.
\end{abstract}

\footnotetext{
*Work supported by General Atomics IR\&D funds.
} 


\section{Overview of the SSX-FRC experiment}

M. R. Brown, ${ }^{1}$ C. D. Cothran, ${ }^{1}$ M. Landreman, ${ }^{1}$ V. S. Lukin, ${ }^{1}$ and M. J. Schaffer ${ }^{2}$

${ }^{1}$ Swarthmore College

${ }^{2}$ General Atomics

\section{Abstract}

The Swarthmore Spheromak Experiment [1] has been modified to produce a Field Reversed Configuration (FRC) by merging two counter-helicity spheromaks. A new, higher aspect ratio flux conserver has been completed $(R=20 \mathrm{~cm}, L=60 \mathrm{~cm})$. The degree of merging will be controlled with a pair of recomnection control coils. The midplane coils are close to the plasma, in the vacuum space between the flux conserver and the vacuum vessel. Complete merging will fully annihilate the original + and - toroidal flux of the spheromaks and produce a conventional FRC. Partial merging will prevent complete toroidal flux annihilation forming a new object called a doublet-FRC.

We have performed experiments using a $3 \mathrm{D}$ magnetic probe array consisting of a $5 \times 5 \times 8$ rectangular grid of 600 coils to measure $B$ at 200 locations with $2 \mathrm{~cm}$ resolution in space and sub- $\mu \mathrm{s}$ resolution in time. In the first set of experiments [2], we studied magnetic structure of local $3 \mathrm{D}$ reconnection in the original SSX configuration allowing only partial merging. Counter-helicity spheromaks merge rapidly, and reconnection activity clearly self-generates a local component of $B$ which breaks the standard 2D symmetry at the ion inertial scale. Consistent with prior esults, no reconnection is observed for co-helicity merging. In a recent campariste field structure consistent with FRC ing of spheromaks into an FRC. Preliminary results

A recent hybrid simulation [3] predicts FRC tilt stabilization due to the spontaneous generation of + and - toroidal flux ropes. We will monitor generation of toroidal flux, as well as global equilibrium and stability characteristics of the FRC with arrays of up to 600 magnetic probes in an azimuthal array dispersed throughout thill be snonitored with Mach probes. Line averaged density (needed to to be driven by flow which will be monitored completed He-Ne laser interferometer system.

compute $s$ ) will be monitored by a spheromak merging

Finally, we have performed axisynmetrict identical global [4]. We will present results from several nacteristics. In particular, comparison between the resistivity $(S=1000)$ but different local chat wiste

[1] M. R. Brown, Phys. Plasnas 6, 1717 (1999).

[2] C. D. Cothran, et al, Phys. Rev. Lett. (submitted).

[3] Yu. A. Omelchenko, Phys. Plasmas 7, 1443 (2000).

[4] V. S. Lukin, et al, Phys. Plasmas 8, 1600 (2001).

Research supported by US DOE grant ER54604 and General Atomics. 


\title{
Comparison of z-pinch and theta-pinch drive for imploding solid liners suitable for compression of Field Reversed Configurations's
}

\author{
J. H. Degnan, P. J. Turchi, and R. E. Siemon (1) \\ Air Force Research Laboratory, Directed Energy Directorate \\ (1) Los Alamos National Laboratory
}

\begin{abstract}
A comparison of Z-pinch and Theta-pinch driven implosions of metal shells (solid liners) is presented. The liners are Al, $30 \mathrm{~cm}$ long, $10 \mathrm{~cm}$ outer diameter, and $\sim 0.1 \mathrm{~cm}$ thick. The circuit parameters are those of the 1300 microfarad Shiva Star capacitor bank, operated at $~ 80$ kilovolts charge. The initial inductance used for this study is 35 to 44 nanohenries. The series resistance includes a safety fuse $\left(2.125 \mathrm{~cm}^{2}\right.$ cross section, 0.94 meter long, Al using a glass bead quench medium) and an external resistance of approximately a milliohm.
\end{abstract}

Both schemes are feasible, and they have different advantages for compression of magnetized plasmas to Magnetized Target Fusion (1) conditions. The Z-pinch approach has already demonstrated at least $35 \%$ conversion efficiency from stored electrical energy (4.4 megajoules) to implosion kinetic energy ( 1.5 megajoules), with good implosion behavior and symmetry, and at least 13 times radial convergence of the liner inner surface (2). The Theta-pinch approach has potential advantages for purer and easier injection of Field Reversed Configurations, easier diagnostic access, and may be more easily operated repetitively. Its calculated conversion efficiency is $\sim 25 \%$, or $\sim 70 \%$ that of the Z-pinch driven approach.

This work is supported by DOE-OFES.

(1) K.F.Schoenberg, R.E. Siemon et al, LA-UR-98-2413, 1998

(2) J.H.Degnan et al, IEEE Transactions on Plasma Science 29, p.93-98 (2001). 


\section{Performance and Stability Limits at Near-unity Aspect Ratio in the Pegasus Toroidal Experiment}

R.J. Fonck, S.J. Diem, G.D. Garstka, A.C. Sontag, K.L. Tritz, E.A. Unterberg, University of Wisconsin-Madison

The accessible parameter space at near-unity aspect ratio in the Pegasus Experiment has been increased. Beta $a_{t}$ values up to $25 \%$ $\left(\right.$ bet $_{N} \sim 5$ ) were obtained with no evidence of a limit, while densities range up to the Greenwald limit. A toroidal field utilization factor up to 1.2 and normalized currents greater than 5 have been achieved. The stored energy is consistent with expectations from the ITER98pby2 scaling. Plasma startup is characterized by high current ramp rates (15-45 MA/s) and low internal inductance $(l i \sim 0.3)$. The current is usually limited by volt-seconds or a large $\mathrm{m} / \mathrm{n}=2 / 1$ MHD instability, which results in a rapid decrease in $d I_{p} / d t$. The appearance of this mode is coincident with $q(0)<=2$ and low shear across a broad region of the plasma interior. A more complete set of magnetic diagnostics was installed to better characterize these plasmas. Increased ohmic power, HHFW pre-heating, variable shaping, improved conditioning and gas handling, and transient high toroidal field during plasma formation provide new tools to control the MHD activity and move closer to near-unity beta plasmas in this geometry.

Supported by U.S. DoE Grant No. DE-FG02-96ER54375 


\title{
Field Reversed Configuration Formation Simulations with MACH2
}

\author{
M.H.Frese and G.J.Marklin, Numerex
}

A Field Reversed Configuration (FRC) plasma is formed by applying a modest magnetic bias field to a gas, then ionizing the gas - perhaps with a high frequency electromagnetic field - and, finally, quickly applying a much larger magnetic field of opposite polarity. The field reversed theta pinch that results can produce magnetically insulated plasma of hundreds of $\mathrm{eV}$ in temperature. Simulations of this experiment can now be performed using the 2 dimensional magnetohydrodynamic code $\mathrm{MACH} 2$. this poster will briefly describe how the code was modified. The poloidal components of the magnetic field are now defined from the flux function which replaces them as the primary state variable and a new finite differencing scheme is used which exactly preserves div(B) $=0$. A critical issue in simulations of this type is the anomalous resistivity model. Spitzer resistivity is never adequate because it invariably leads to hot, highly conducting vacuum regions, which prevent electric fields from diffusing into the plasma. Past FRC experiments at LANL have shown that the Chodura model with empirically determined parameters has led to good agreement with simulations [1], so this model, with their parameters, was used. Three runs were done using this model, with three different initial densities. Additional runs used the lower hybrid model with MACH2's default parameters, and the Spitzer model with Ohmic heating turned off in low density regions. Contour plots are shown of the flux, density, and temperature as a function of time using the different resitivity models. The maximum temperature is weakly dependent on the resistivity model, and strongly dependent on the initial density.

References:

This material is based on work supported by the United States Air Force, and by DOEOFES.

Footnotes:

[1] Brackbill and Milroy, Physics of Fluids, May 1982 


\section{Equilibrium and Stability Analysis of Pegasus Plasmas}

G.D. Garstka, S.J. Diem, R.J. Fonck, A.C. Sontag, K.L. Tritz, E.A. Unterberg, University of Wisconsin-Madison

Magnetic equilibrium analyses of near-unity aspect-ratio discharges in the PEGASUS Toroidal Experiment have been performed using a locally developed code which incorporates a nonlinear least-squares fitting routine coupled to a Grad-Shafranov solver. Induced currents in the continuous, resistive vessel wall are estimated using a time-evolving current filament model and are constrained during the reconstruction by wall-mounted flux loops and B-dot coils. With $I_{\text {wall }} / I_{p}$ up to 2 , the wall contribution to the total poloidal field often dominates early in the discharge. An upgrade of the internal magnetics set to include 20 poloidal flux loops, a poloidal array of 20 B-dot coils, and a diamagnetic loop has increased the accuracy of equilibrium reconstructions. Plasmas with $A<1.3$, $I_{p}<=0.15 M A, 0.2<l_{i}<0.8$, and beta $a_{t}<25 \%$ have been analyzed. The presence of a broad $q \sim 2$ region inside the plasma corresponds to the growth of a large $m=2 / n=1$ internal mode; at higher values of plasma current an $m=3 / n=2$ mode has been observed. Ideal stability analyses have been performed using DCON; these analyses predict instability to external kink modes in good agreement with observed plasma disruptions.

Supported by U.S. DoE Grant No. DE-FG02-96ER54375 


\section{Equilibrium of the Electric Tokamak*}

J.-L. Gauvreau, P.-A. Gourdain, D.J. Lafonteese, L.W. Schmitz and
R.J. Taylor, University of California, Los Angeles (UCLA), CA 90024

The Electric Tokamak (ET) is now operating at .25 Tesla with $60 \mathrm{kA}$ of plasma current and with a pulse length as long as $2.5 \mathrm{sec}$. The current as well as the radial and vertical positions are controlled in real-time, giving a stable target for ICRH at the 2nd harmonic. Discharge cleaning is done overnight and Titanium gettering on the walls is done on a daily basis to insure excellent wall conditions and $Z_{\ldots}\{$ eff $\}<1.1$. V_L of $<.6$ Volt is achieved in ohmic conditions. Without Ti deposition, V_L $>1$ Volt and semidisruptions caused by internal kink modes become frequent. With about 50 $\mathrm{kW}$ of RF heating, the loop voltage decreases by $30 \%$ and sawtoothing disappears, indicating a broadening of the current profile. We are in the process of increasing the RF input power to the $2 \mathrm{MW}$ level. The effect of such power on the loop voltage and the ion temperature will be presented. Stability analysis is performed using DCON and NIMROD codes. We are experimenting with plasma elongation to improve antenna coupling. We are also preparing higher harmonic current drive for long pulse operation (30 sec) and for shaping the current profile to peak near the outer edge. This profile will help avoid low n,m internal kink modes and the longer pulses will allow plasma current relaxation to take place.

*This Research Supported by US DOE grant DE-FG03-86ER53225 


\section{Crowbar Switch for Configuration Formation Field Reversed}

T.C. Grabowski, T.C. Cavazos, D.G. Gale, W.E. Sommars, J.H. Degnan (1), T. P. Intrator (2), R.E. Siemon (2), J. M. Taccetti (2), B. Waganaar (2), and G. A. Wurden (2)

SAIC

(1) Air Force Research Laboratory, Directed Energy Directorate

(2) Los Alamos National Laboratory

The formation of a Field Reversed Configuration (FRC) suitable for compression to Magnetized Target Fusion (MTF) conditions [1] requires at least 3 discharges. These include (1) a slow risetime discharge (> 100 microseconds) to establish an $\sim 0.4$ Tesla axial magnetic field in a $10 \mathrm{~cm}$ diameter, $30 \mathrm{~cm}$ long theta discharge tube with $\sim 50$ milliTorr deuterium prefill; (2) a faster, modest energy, oscillating pre-ionization discharge, with an amplitude comparable to the bias field; and (3) a more energetic reverse-field discharge with a risetime of 2 to 3 microseconds, a longer decay time, and an amplitude $\sim$ factor of 10 greater than the initial bias field. A Shiva Star module drives this third discharge, which must be crowbarred so that the FRC will last long enough (10 to 20 microseconds) for subsequent translation and compression.

This presentation reports the crowbar switch development. The transmission line connecting the Shiva Star module to the theta discharge coil includes a section that converts from parallel plate to an array of 48 coaxial cables. The crowbar shorts out the cable connections when the discharge current is near maximum. This has been designed, built, assembled, and tested at the Air Force Research Laboratory. It has been operated successfully for 80 kilovolt, $\sim 1$ megamp discharges into a $40 \mathrm{nH}$ theta coil. The oscillations in the crowbarred current are approximately $+/-20 \%$. The crowbar switch is a pressurized railgap switch very similar to those used as closing switches. Tests with a pre-ionization-like discharge driving the same theta coil indicate no pre-triggering of the main reverse-field discharge. This switch is now mounted on the Colt capacitor bank (a single Shiva Star module) and integrated with the rest of the FRC experiment hardware at Los Alamos.

[1] K.F.Schoenberg, R.E. Siemon et al, LA-UR-98-2413, 1998 


\section{Convective Cell Formation in a Magnetic Dipole Configuration}

Priyanka Goswami, A. M. Rey, W. Dorland, and A. B. Hassam Institute for Research in Electronics and Applied Physics University of Maryland

Convective cell formation for a magnetic dipole configuration is being studied. Close to the axis, the dipole configuration can be considered to be a Zpinch. In a Z-pinch the convection of the plasma results from asymmetric heat and particle sources. It was found that there is no convection if there are no particle sources, independent of the distribution of the heat sources. Particle sources result in convection which in turn influence heat transport. Our analysis closely follows the calculation of the convective cell formation in a Z-pinch. The work for dipole configuration is of interest because of the recent LDX experiment. 

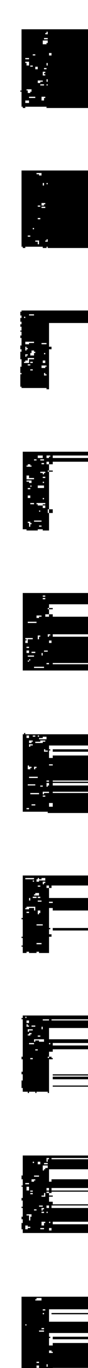

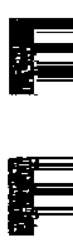

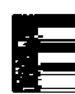

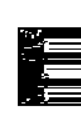

$E$

E

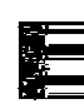




\section{Ion ring formation by radial injection}

\section{J.B. Greenly, W.J. Podulka, S.C. Glidden, Cornell University}

A first set of experiments has been completed on the Cornell ion ring experiment FIREX, with a novel ion diode. The diode is coaxial with the anode at $5 \mathrm{~cm}$ radius and the cathode at $6 \mathrm{~cm}$ radius from the axis of a solenoidal magnetic field. The diode accelerates an intense proton beam radially outward. The object is to use the solenoidal field to confine this beam to form a strong ring. The field $(0.6 \mathrm{~T})$ also provides magnetic insulation of the diode accelerating gap. The anode is composed of axially streaming hydrogen plasma produced by an inductive gas-breakdown source inside the anode (high-voltage) feed of the $700 \mathrm{kV}$ FIREX pulser. The cathode is a drifting electron population emitted from the end of the cathode of the biconic MITL feed. First results showed that this diode and feed operate with good efficiency and produces 50-100 A/cm2 proton current density over $\sim 40 \mathrm{~cm}$ axial length $(\sim 1500 \mathrm{~cm} 2$ area) with excellent uniformity. This represents a possible factor of two increase in the proton population for ring formation in FIREX, with favorable stability properties. 


\title{
Simulations of ion ring dynamics
}

\author{
A.V. Gretchikha, J.B. Greenly, Yu.A. Omelchenko, Cornell \\ University
}

Sensitivity of Alfven wave dynamics to changes in the ion ring and background plasma in the FIREX experiment are being studied in simulations with the 2D code FIRE in both $(r, z)$ and $(r$,theta) geometry. Comparisons are made between the experimental Bdot probe measurements and the magnetic fields in the simulations. Diagnostics of ring proton orbits are also compared with simulations. Both experimental rings and simulations show instability with loss of ring energy to alfven waves. Factors affecting stability are being clarified. Regimes in simulation have been found which show relaxation from the very unstable,nearly singular injected ring distribution to more disordered states, driven by instability, and followed by stable, slow resistive decay. Differing amounts of ring energy are lost in the relaxation depending on the initial ring distribution. The results indicate that the standard FIREX cusp injection may be much less favorable than the new radial injection. 
FRC Translation and Capture Measurements on TCS. J.A. Grossnickle, H.Y. Guo, A.L. Hoffman, J.T. Slough. University of Washington

Field Reversed Configurations (FRC) have been formed, accelerated to high velocity, and captured in the TCS device. This is necessary in order to achieve a hot $(\sim 500 \mathrm{eV})$, low density $\left(\sim 10^{20} \mathrm{~m}^{-3}\right)$ FRC for Rotating Magnetic Field (RMF) current drive studies. Approximately $\mathrm{n}_{\mathrm{e}} \sim 10^{21} \mathrm{~m}^{-3}, \mathrm{~T}_{\mathrm{e}}+\mathrm{T}_{\mathrm{i}} \sim 500 \mathrm{eV}$ FRCs are formed in the $40 \mathrm{~cm}$ diameter $\mathrm{LSX} / \mathrm{mod}$ device at about $1 \mathrm{~T}$ fields, ejected through a smaller $27 \mathrm{~cm}$ diameter translation section (previously used for acceleration experiments), and then expanded into the much larger $80 \mathrm{~cm}$ diameter by $3 \mathrm{~m}$ long TCS quartz chamber. Upon translation into the $0.2 \mathrm{~T}$ translation section and 0.1 T TCS chamber; the FRCs cool adiabatically and reach highly super-Alfvénic speeds. This velocity is lost upon several reflections off the TCS end mirrors and much of the original temperature is recovered. A testament to the FRC's robustness (at least at low $\mathrm{s}$ ) is the ability to recover from such violent deceleration, and form an FRC that has even better lifetimes than predicted by previous high-density empirical scaling laws. A remarkable feature of the process is an apparent increase in poloidal flux after the initial reflection, which is not presently understood. Detailed studies of the FRC poloidal and toroidal fields, using a set of internal B probes, will be used to determine the origin of this flux enhancement. One previous problem has been impurity pickup upon reflection from the conical stainless steel end of the TCS chamber. A Tantalum shield has been installed over the stainless steel to reduce this impurity pickup. RMF drive experiments on the translated FRCs will begin once the initial conditions are optimized. 
Formation and steady state maintenance of FRCs using Rotating Magnetic Field (RMF) current drive in the TCS experiment. H.Y. Guo, R.D. Brooks, A.L. Hoffman, Z.A. Pietrzyk, University of Washington. The Translation, Confinement, and Sustainment (TCS) experiment was designed to demonstrate flux build-up and sustainment of standard, elongated, flux confined FRCs. The ability to overcome initial radiation barriers was provided by attachment to the LSX/mod theta pinch device, so that hot FRCs could be translated and expanded into TCS. Initial experiments have been preformed in TCS using RMF formation alone from preionized gas fills. Although no fueling was provided for in the initial phase of the experiments, quasi steady-state (flux, particles, and energy) FRCs were produced for the length of the RMF pulse (about 2 $\mathrm{msec}$ ). Up to $60 \mathrm{kA}$ of toroidal current was maintained by RMF fields of about $60 \mathrm{G}$. The RMF not only maintains the FRC flux, but also provides a strong radial inflow that results in a near zero density at the separatrix and prevents particle loss over that portion of the FRC under the RMF antennas. For cases where the maximum possible synchronous line current, $I_{s y n c}^{\prime}=0.5\left\langle n_{\mathrm{e}}\right\rangle e \omega r_{\mathrm{s}}{ }^{2}$ exceeds the equilibrium reversal line current $I_{r e v}^{\prime}=2 B_{\mathrm{e}} / \mu_{0}$, (represented by the parameter $\zeta=I_{r e v}^{\prime} / I_{s y n c}^{\prime}$ ), the RMF only partially penetrates the FRC, in agreement with simple numerical models. Experiments with initial high RMF frequency $\left(\omega=1.0 \times 10^{6} \mathrm{rad} / \mathrm{s}\right)$ had relatively low values of $\zeta$ and the current was primarily edge driven. The drive efficiency was relatively low and could lead to unsteady behavior as a field null could be formed too close to the separatrix. When the RMF frequency was lowered to $\omega=0.5 \times 10^{6} \mathrm{rad} / \mathrm{s}, \zeta$ increased, allowing higher densities $\left(n_{\mathrm{e}} \sim 3 \times 10^{19} \mathrm{~m}^{-3}\right)$ to be produced and resulting in steady behavior for over ten normal flux lifetimes of similar unsustained FRCs. The plasma temperature cannot be increased much above $50 \mathrm{eV}$ for these purely RMF formed FRCs, and the cross-field resistivity is high, This would be expected to lead to rapid ion spin-up, but the spin-up was observed to saturate at about $0.2 \omega$. It is thought that this is due to ion-neutral friction, although the calculated necessary neutral fraction is considerably higher than estimated from global power balance. Nevertheless, it is expected that some form of counter momentum injection, i.e. from tangentially injected neutral beams, will be desirable for future facilities. Experiments have begun on translating and capturing hot $(\sim 300 \mathrm{eV})$, theta pinch formed FRCs, which exhibit much lower resistivities. As soon as initial densities can be lowered slightly, RMF flux build-up and sustainment experiments will begin in this more interesting plasma regime. 


\title{
Status of the Maryland Centrifugal Experiment(MCX)
}

\author{
D.K. Gupta, R.F. Ellis, A.B. Hassam, S. Messer, University of \\ Maryland
}

The Maryland Centrifugal Experement is in the midst of the construction phase. The purpose of MCX is to test centrifugal confinement of plasmas and velocity shear stabilization of MHD interchange instabilities. The geometry of the magnetic field is that of a solenoid with axisymmetric mirror end fields. Biasing of an inner core relative to the outer wall produces a radial electric field which will drive supersonic ExB azimuthal rotation and the resulting centrifugal force will contain plasma to the solenoidal portion. On MCX the solenoidal field is produced by two 25 inch ID coils with a maximum strength of $0.5 \mathrm{~T}$ and the mirror fields are produced by four 18 inch ID coils with a maximum field of $1.8 \mathrm{~T}$; the mirror ratio is variable from $3-10$. The mirror and solenoidal coils have been installed and completely tested and meet specifications. A future upgrade will incorporate a toroidal magnetic field with a strength at midplane up to $0.3 \mathrm{~T}$. The vacuum vessel is $55 \mathrm{~cm}$ diameter at midplane with an overall axial length of $410 \mathrm{~cm}$. The vessel consists of three sections to allow ease of access and has multiple diagnostic ports. The vessel has been delivered and will be installed in the system by February. The plasma will have a radial width of $15-20 \mathrm{~cm}$ at midplane and an elongation of 5-10. A $10 \mathrm{KV}$, 0.4 MJ capacitor bank has been constructed as the power source, with upgrade capability to $18 \mathrm{KV}$ and $1.5 \mathrm{MJ}$. Preionization sources are being studied. Plasma densities in the range (1-10) $10^{13} \mathrm{~cm}-3$, temperatures from 10-50 eV, and Mach numbers greater than 2 are expected. 


\section{Physics and Operations Plan for LDX}

A. Hansen, D. T. Garnier, M. E. Mauel, T. Sunn Pedersen, E. Ortiz, Columbia University

J. Kesner, C. M. Jones, I. Karim, J. Minervini,

P. Michael, A. Zhukovsky, MIT Plasma Science and Fusion Center

Immediately after construction, the Levitated Dipole Experiment (LDX) will begin its plan to study the confinement and stability of high-temperature plasma in dipole geometry. The primary objective of this program is to investigate the possibility of steady state, high-beta dipole confinement with near classical energy confinement. The experimental program will also include new studies of basic and applied plasma science including: (1) the study of high beta plasma stabilized by compressibility, (2) the relationship between drift-stationary profiles having absolute interchange stability and the elimination of drift-wave turbulence, (3) the induced flows and characteristics of lowfrequency electrostatic potential structures, or convective cells, (4) the coupling between the scrape-off-layer and the confinement and stability of a high-temperature core plasma, (5) the stability and dynamics of high-beta, energetic particles in dipolar magnetic fields, and (6) the long-time (near steady-state) evolution of high-temperature magneticallyconfined plasma.

In order to achieve these goals, we have developed a staged physics and operation plan that both establishes the operation of LDX and minimizes risks to the superconducting coil systems. The initial experiments will feature the dipole field coil supported within the vacuum chamber, rather than levitated. This configuration has end losses for magnetic field lines that intersect the supports, and consequently provides benchmark plasmas for comparison with levitated coil operation.

LDX plasmas will be formed and heated using ECRH. Initially, $3 \mathrm{~kW}$ will be applied at $6.4 \mathrm{GHz}$, because that source is currently operational. Eventually power will be applied at multiple frequencies, i.e. multiple resonance regions. We plan to use this multiple-frequency capability to control the heating (and pressure) profile by adjusting the input power from each frequency. Profiles adjusted to be near marginal stability should maximize $\square\langle\beta\rangle$ We also plan experiments that adjust the axial symmetry of the heating and fueling in order to produce and/or suppress convective cells. Control of the pressure profile will also help reduce convective energy losses. When the pressure profile has gradients near critical, these so-called "stationary profiles" minimize thermal convective-cell transport associated with particle circulation.

Only base-case diagnostics will be employed for the first plasmas. The initial magnetic diagnostics set will include flux loops, Hall probes, and Mirnov coils. The edge magnetics will be employed for fluctuation measurements as well as for equilibrium reconstruction. X-ray and microwave diagnostics will be used to measure the energy of the energetic electrons and the total electron density profile. External coils will be switched on to change the magnetic topology and directly test high-beta plasma stability due to compressibility effects. 


\section{Modeling SSPX Formation and Equilibria \\ E. B. Hooper, S. Woodruff, B. I. Cohen, and the SSPX Team* \\ Livermore, CA 94551}

examine associated physics issues. The CalTrans (Corsica) code calculates axisymmetric,
op MHD equilibrium to design experiments and fit magnetic probe data [1] equilibria, are simulated by pendent effects. Current-drive and trabe data [1]. It can also be assumed approximately closed. Cuced models in the presence of fluxsport, and their effects on law. The NIMROD code [2] is a 3Dent drive results from a hyper-surfaces which are magnetic reconnection; usually a 3D, resistive-MHD code whyper-resistive term in Ohm's about flux surfaces, and in mally it uses a one-fluid approximatich includes the effects of present. The code describes thest calculations the fieldintines arcon. No assumption is made current injected from the "

This poster compares ren," and includes fluid flow ond the spheromak, starting with the of using the two approxim results from the two codes and related physics.

1) Mean magnetic fields sogether to understand the the experiment, with the goal agrees well with CalTrans, as. The $n=0$ component of the spheromak physics. be interpreted as a "mean" as do the n=0 components of $q$ and $\lambda=N$ iMROD calculations open with average lengn" field despite the fact that fiel $q$ and $\lambda=j \bullet B / B$. This field cons

2) Evidence fongths only a few circumference of tines as calculated by field can thus gun $\lambda \approx \lambda_{F C}$, where $\lambda$ against closed magnetic surfaces the flux conserver. $\nabla \times B=\lambda_{F C} B$, the $\lambda_{F C}$ is the eigenvalue for surfaces. In experimental

values on either electron temperature and the first resonance of the foperation with the The experimer side of the axis are on ind density are peaked on the flux conserver, data, and we col magnetic fluctuations antical values of the flux calculagnetic axis and the However, whenclude that closed flux surf small $(\sim 1-2 \%)$ and have low caled by CalTrans. Thomson when the gun current is raised, the fluctuations become firt approximation.
that fieldlines are the

3) Current open as predicted by NIMROD. which can be conive. The hyper-resistive curient low fluctuations. NIMROD wheory. The results are prive model uses an ad hoc coefficient comparison betwinterior field measurements in $\lambda$-profiles which show "patchy" spatial with

4) Temperatures. Rechester-Rosenblus. The measured electron temperath electron betas contuth model for transport Howeratures in SSPX are are being includedsistent with experiment. However, the NIMROD code consistent with a comparison with in the NIMROD calculation, effects of open fieldline also generates "This work was prich will permit a more explicit

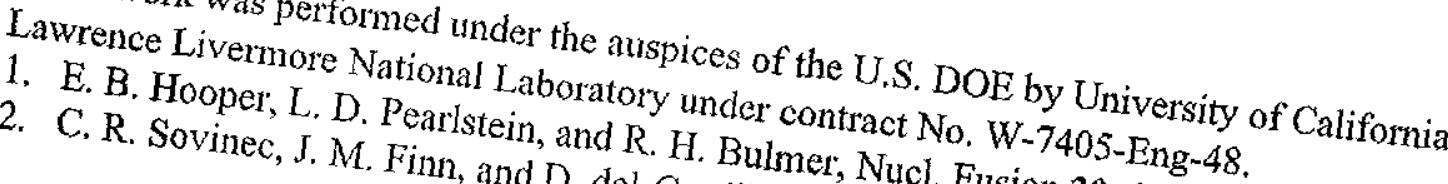

C. R. Sovinec, J. M. Finn, and D. del-Castillo-Negrete. Fusion 39, 863 (1999). 
Experimental Study of Spheromak Formation Using a Planar Coaxial Gun, S. C. Hsu and P. M. Bellan, California Institute of Technology

We have constructed the simplest possible coaxial helicity source, a planar disk-annulus electrode setup, in order to study spheromak formation in a clean manner. The issues we are studying include gas injection, gun efficiency and impedance, helicity injection, and the non-axisymmetric instabilities required for spheromak formation. Using a state-of-the-art multi-frame CCD camera, we have obtained high-quality images of the spheromak formation process. The plasma evolution is most strongly determined by the Taylorstate eigenvalue $\lambda_{\text {gun }}=\mu_{0} I_{\text {gun }} / \psi_{\text {gun }}$ imposed at the gun (where $I_{\text {gun }}$ is the gun current and $\psi_{\text {gun }}$ is the initial poloidal flux intercepting the inner gun electrode). For low values of $\lambda_{\text {gun }}$, a plasma column forms along the geometric axis and persists for many Alfvén times. For intermediate values of $\lambda_{\text {gun }}$, the central column develops a highly coherent and nonlinear helical instability when $\lambda_{\text {gun }} L \gtrsim 4 \pi$, where $L$ is the column length. This condition is equivalent within a constant of order unity to the Kruskal-Shafranov condition $q(a)>2 \pi a B_{Z}(a) / L B_{\phi}(a)$, where $q$ is the safety factor and $a$ is the column radius. For high values of $\lambda_{\text {gun }}$, the plasma detaches quickly and propagates along the geometric axis on the order of the Alfvén speed. The planar gun impedance is nominally $30 \mathrm{~m} \Omega$, about three times larger than that of a predecessor cylindrical gun ${ }^{1}$ using the same power supplies. This is thought to be due in part to the elimination of the gun "coupling region," resulting in higher mass flow and therefore higher inductive $V_{\text {gun }}$ and also higher helicity injection rate $\dot{K}=2 V_{\text {gun }} \psi_{\text {gun }}$. Parameters are as follows: $n_{\mathrm{e}} \sim 10^{14} \mathrm{~cm}^{3}$, $T_{\mathrm{e}} \approx 10-20 \mathrm{eV}, I_{\text {gun }} \approx 100 \mathrm{kA}, B \sim 0.2-1 \mathrm{kG}, V_{\text {gun }} \approx 2 \mathrm{kV}, \psi_{\text {gun }} \approx 1-2 \mathrm{mWb}$, $L \approx 30 \mathrm{~cm}, V_{\mathrm{A}} \sim 50 \mathrm{~km} / \mathrm{s}, \rho_{\mathrm{i}} \sim 1 \mathrm{~cm}$, and $S \sim 10^{2}-10^{3}$. Because our gun boundary conditions approximate those of a magnetized star-disk system, we are also investigating the relationship of our configuration to accretion disks and astrophysical jets. The formation of a stable central column in our experiment supports the concept of magnetically-driven jets and winds. A magnetic probe array will be installed in the near future to obtain localized magnetic field vector components.

\footnotetext{
${ }^{1}$ Yee and Bellan, Phys. Plasmas 7, 3625 (2000).
} 


\title{
NMCX: Numerical Maryland Centrifugal Experiment
}

\author{
Y.M. Huang, A.B. Hassam, R.F. Ellis, IREAP, University of \\ Maryland
}

MHD stability is a key issue of a centrifugally confined fusion device. In this paper, a magnetized, centrifugally confined plasma was numerically shown to be stable on account of velocity shear, and a laminar profile was maintained. As a comparison, we set up a new system wherein the plasma was confined by a fake gravitational field instead of the centrifugal force, and a extra heating was added in to maintain the temperature. This new system was observed to be grossly unstable to the "flute" interchange modes. The turbulent plasma was then fed into the original system, and a laminar profile was recovered by the imposed velocity shear. This opens up the possibility of a magnetically confined plasma for thermonuclear fusion that has a particular simple coil configuration. 


\section{Simulation of Inductive Startup of a Spherical Torus without using an $\mathrm{OH}$ Solenoid}

S.C. Jardin, PPPL

Several methods have been proposed for the startup of an ST without using an $\mathrm{OH}$ solenoid between the plasma and the inner TF leg. One method, being explored in NSTX, is to drive a coaxial current (CHI) across an insulating slit in the vessel, and then to rely on plasma instabilities to rearrange the configuration to create nested flux surfaces with current that do not intersect the vessel walls. Another method, which is a variation on this method, is to create a high current arc using CHI, in such a way that a field null exists in the vacuum vessel. Abruptly terminating this arc will be like a "plasma OH-coil" and can induce a current at the field null.[1] A third method requires internal PF coils as in the MAST experiment. The plasma can be formed around the internal coils, merged together, and compressed. Here we focus on a 4th method that involves an external PF coil, an insulating break, and electrodes. The electrodes draw a poloidal current across the break, which develops a toroidal component (in order to remain force-free). This is sufficient to produce an o-point just inside the vessel. Subsequent ramping down and reversing the outer midplane $\mathrm{PF}$ coil will induce a large toroidal current and nested closed field lines in the plasma. TSC simulations are presented to show how the current profile in the final configuration depends on the strength and timing of the electrode current and external PF ramp.

[1] This method has been independently proposed by L.Zakharov 


\title{
Theory of Plasma Confinement in a Levitated Dipole
}

\author{
J. Kesner \\ MIT Plasma Science and Fusion Center \\ D.T. Garnier. A. Hansen, M.E. Mauel \\ Columbia University
}

Plasma confinement in the field of a levitated ring offers a new and exciting approach for magnetic confinement. As observed in space plasmas a dipole confined plasma is intrinsically steady state and can have $\beta>1$. In a laboratory plasma, losses to supports can be eliminated by levitating a superconducting coil within a vacuum chamber. Compared with a tokamak, a dipole plasma does not require current drive and is free of disruptions. Theoretical studies indicate the following:

- In the "inner" good-curvature region between the internal ring and the pressure peak the plasma is MIID stable. However, drift waves can be present in this region.

- In 1.le "outer" region between the pressure peak and the limiter (or separatrix) the plasma will be stable to both MHD interchange and ballooning when $\delta\left(p l^{\circ}\right) \geq 0: l^{\circ}=\oint d \ell / B, \gamma=5 / 3$.

- In the outer region drift wave instability can exist for $\eta<2 / 3(\eta=$ $d \ln T / d n n)$. Drift wave stability boundaries are relatively independent of plasma collisionality regime.

- In the outer region convective cells can exist. They can give rise to nonlocal thermal transport. When $p I^{r \gamma} \approx$ constant the flow is adiabatic and only transports particles (not energy).

These results indicate that a dipole confined plasma can have near-classical confinement, exhibit particle self-fueling without energy degradation and support high 3. The pressure gradient limitation requires that a dipole confinement device consists of a superconducting magnet that floats freely within a relatively large vacuum chamber. 
Field Gradient Dependence for Fusion Self-Heating in MTF Targets

Ronald C. Kirkpatrick

Los Alamos National Laboratory

Abstract: The lack of high areal density $(\rho R)$ is augmented by an increased field times radius product (BR) for magnetized target fusion (MTF) targets. It has been shown that for $B R>1 \mathrm{Tm}$ the energy deposition in the target is substantially increased over the zero field value. However, the increase is dependent not only on field geometry, but also on the field gradients. Results for a static azimuthal field having various gradients will be presented.

LAUR-01-6763 
PLANNED MHD STABILITY STUDIES OF CURRENT-CARRYING PLASMAS IN THE COMPACT TOROIDAL HYBRID S.F. Knowlton, G.J. Hartwell, J.D. Hanson (Auburn University, Auburn, AL) The Compact Toroidal Hybrid (CTH) will investigate MHD instability and disruptions in current-carrying compact stellarator plasmas as part of a national compact stellarator program. The central scientific issue is the effectiveness of the external helical field in passively suppressing and/or mitigating major disruptions in current-carrying toroidal plasmas. CTH is presently under construction, and is expected to begin operation in 2003 . The experiment will use ohmic current to investigate both ideal (vertical and kink) and resistive current-driven instabilities in a low aspect ratio $\left(A_{p}=4\right)$, low- $\beta$ stellarator. The configuration of CTH is highly flexible, with the edge vacuum rotational transform variable from $\mathbf{t}_{v}(a)=0.2$ to 0.5 , and the currentgenerated transform $\mathfrak{t}_{f}(a)>0.5$. The experimental coil configuration is being designed to tolerances of $: \pm 1 \mathrm{~mm}$ to reduce static magnetic island sizes to negligible levels. Error correction coils for additional island control are also under design. Stability will be investigated with ohmic currents $I_{p} \leq 60 \mathrm{kA}$ applied to plasmas in which both peaked and hollow, bootstrap-like current profiles will be generated. Target plasmas will be generated by ICRF using a Nagoya Type-III antenna. The expected RF power is $\mathrm{P}_{\mathrm{RF}} \cong$ $250 \mathrm{~kW}$ at $\mathrm{f} \leq 12 \mathrm{MHz}$. Diagnostic plans include the application of an MSE/LIF system for measurement of the internal rotational transform to contribute to the reconstruction of the 3-D plasma equilibrium from magnetic loops. Supported by US DoE Grants DE-FG02-98ER54470 and DE-FG02-00ER54610 


\section{Spectroscopic Diagnostics of Plasma Response to Ion Ring Dynamics}

B. Knyazev, J.B. Greenly, D.D. Lee, W.J. Podulka, Cornell University

Two new spectroscopic diagnostic systems have been developed for the Cornell ion ring experiment, FIREX. First, a MCP-intensified, wide-dynamic rnage CCD is used to record streaked spectral lines from a $1 \mathrm{~m}$ spectrometer. With sub-A resolution and submicrosecond time resolution, plasma ion temperature can be observed by line broadening. The ion rings being formed in FIREX produce largeamplitude Alfven waves near the ion cyclotron frequency. These waves, carrying hundreds of joules heating of the plasma ions will be presented. The second diagnostic is a novel large-aperture spatial Fourier spectrometer, also with MCP-CCD output and computerized data analysis, for general survey spectrometry. 


\title{
The Engineering Physics of an Optimized Confinement Concept (The Plasmak)
}

\author{
P.M. Koloc, J.A. Bowery, R.M. Fujii, C.F. Harrison, \\ W.D. Jackson, A. Lowrey III, and R.B. Pittman
}

We began inventing a potentially best workable concept for fusion in $1973^{1}$, and have since continued that effort from just beyond the community's mainstream. This effort, including experiments, is summarized, and more important advanced features are noted. The topology is Spheromak with fully compressible and impervious conducting shell. What makes the embodiment unique is its self-generated construction that forms the magnetized structure of hyperconducting plasma (no rigid matter). The conceptual and then physical formation of the Plasmak was very difficult to solve. The formation mechanism will be briefly described.

The next experimental effort will be to form with substantially higher energy $(25 \mathrm{~cm}$ diameter at STP in atmospheric air) and with a fully viable lifetime of one to five seconds. The capacitor storage bank will be operated at 12 to $16 \mathrm{kV}$, and, the use of a large solid-state crowbar will allow for longer and more reliable runaway electron currents acceleration time.

If formed of fusionable fuel, the object is ideally suited as an MTF target that is capable of being mechanically fluid compression heated within a boron burn chamber. The system's compactness, simplicity, and efficiency may allow cycled operation at sixty Hertz. Direct injection of the aneutronically heated compression blanket through an Inductive MHD generator will produce electric power with exceptionally high efficiency.

\footnotetext{
1. P. M. Koloc, "Method and Apparatus for Utilizing a Compound Plasma Configuration," U.S. Patent No. 4,023,065, issued 10 May 1977.
} 
The PLASMAK ${ }^{\mathrm{TM}}$ power generator system will be comprised of a formation chamber, a $25 \mathrm{kbar}$ ram fluid-driven compression burn chamber, and an Inductive MHD electric converter that operates at $60 \mathrm{HZ}$. At the core of the system is a fully compressible, highly stable magnetoplasmoid (PMK) that has colossal ruggedness due to its hyperconductivity and high current density ("g" is about a third of superconductivity on the log scale). While compact toroids have similar topologies, the formation, physical embodiment, and fluid mechanical compression to ignition of the PMK are an elegant symphony of engineering physics. For example, the PMK will self generate in a one or two bar blanket of $\mathrm{p}^{-11} \mathrm{~B}$ due to a single injection of helicity from a modified button gun. Energetic runaways are accelerated when the central magnetized stem channel that connects the surrounding axisymmetric sheath with a central electrode begins to kink and form a tightly wound helix, which then coalesces into a magnetized torus and insulating field in the central midplane. Complete descriptions will be addressed in the presentation. We have been blessed to have captured, sometimes serendipitously, a large cross section of the PMK's unique, and occasionally spectacular, characteristics. Our experimentally produced atmospheric plasmoids have steady lifetimes that are 10,000 times longer than typical Spheromak lifetimes. Our laboratory is a skunkworks, and our work is self-funded. Our next development will be to form atmospheric PMKs of $\sim 25 \mathrm{~cm}$ (much larger) diameter, and with steady lifetimes from 1 to 5 seconds. 


\section{Exhausting plasma to outside the TF coils}

\section{Kotschenreuther, IFS}

Here we show that it is possible to exhaust the separatrix plasma of toroidal magnetic configurations (e.g. tokamaks) to outside the TF coils without contacting material surfaces. This can produce a very hot separatrix plasma, resulting in a new confinement regime and enhanced energy confinemnt, possibly up to several times standard operation. Innvative energy conversion options become possible and will be discussed, including deuterium based fuel cycles with direct conversion and D-He3 interplanetary propulsion with an ST. For DT operation, serious engineering problems due to heat loads on the divertor and first wall can be aleviated, enabling higher power density. 


\section{Muon Catalyzed Fusion Revisited}

R.M. Kulsrud, Princeton Plasma Physic Laboratory

One way to make power out of muon fusion is to extend the number of muons catalyzed by one muon beyond the limit given by trapping into an He-muon ion. This can be done by fragmenting the target into thin slabs (100 microns) and keeping the He-muon ion going by cyclotron acceleration in between the slabs. Up to a thousand fusions can be catalyzed by fusion using this method but the gain is nil because the fusions melt the slabs at low temperature and one loses on thermodynamic grounds. This can be cured by allowing a rapid expansion of the slabs as the fusion goes on and due to the fusion energy. The consequence of all this is that the fusion gain is limited by the molecular formation rate. Up until last year this was believed to be too slow for a net gain of the fusion energy over the cost of the slabs plus the cost of the muon. Last year Fujiwara et al. actually measured the molecular formation rate, and found it to be higher by up to a factor of seven due to a resonance in the formation rate that occurs at 5000 degrees Kelvin. With this faster rate the fusion $Q$, the fusion power (thermal) divided by the cost of the slabs plus the muons is at least as great as four. A more skillful placement of the slabs could increase this still further. The implications of all this will be discussed.

Fujiwara et. al. Phys. Rev. Lett. 85, 1642,2000 


\section{PHYSICS OVERVIEW OF THE QPS EXPERIMENT}

\section{J.F. Lyon, Oak Ridge National Laboratory, representing the QPS team}

The Quasi-Poloidal Stellarator (QPS) is being developed to study very-low-aspect-ratio $(\mathrm{R} / \mathrm{a}=$ 2.7) compact stellarators in which the dominant components in the magnetic field spectrum are poloidally symmetric in flux coordinates. This leads to small B $x$ grad B drifts out of a flux surface and reduced neoclassical transport at low collisionality. The magnetic configuration may also have reduced poloidal viscosity, allowing larger poloidal flows, and relative insensitivity of the magnetic configuration to increasing beta. QPS has $<\mathrm{R}>=0.9 \mathrm{~m},<\mathrm{a}>=0.33 \mathrm{~m},<\mathrm{B}_{\text {axis }}>=$ $1 \mathrm{~T}$ for a 1-s pulse, and $\mathrm{P}$ (heating) $=1-3 \mathrm{MW}$. The experiment is designed to study regimes in which either anomalous transport or neoclassical transport is dominant and to test ballooning stability limits at $\langle\beta\rangle=2.5 \%$, the configuration dependence of the bootstrap current, and equilibrium robustness at finite beta. The poster will address the physics basis for the QPS approach, the relationship with other stellarator concepts, the key features of the proposed QPS experiment, and the connection to higher-beta quasi-poloidal-stellarator configurations.
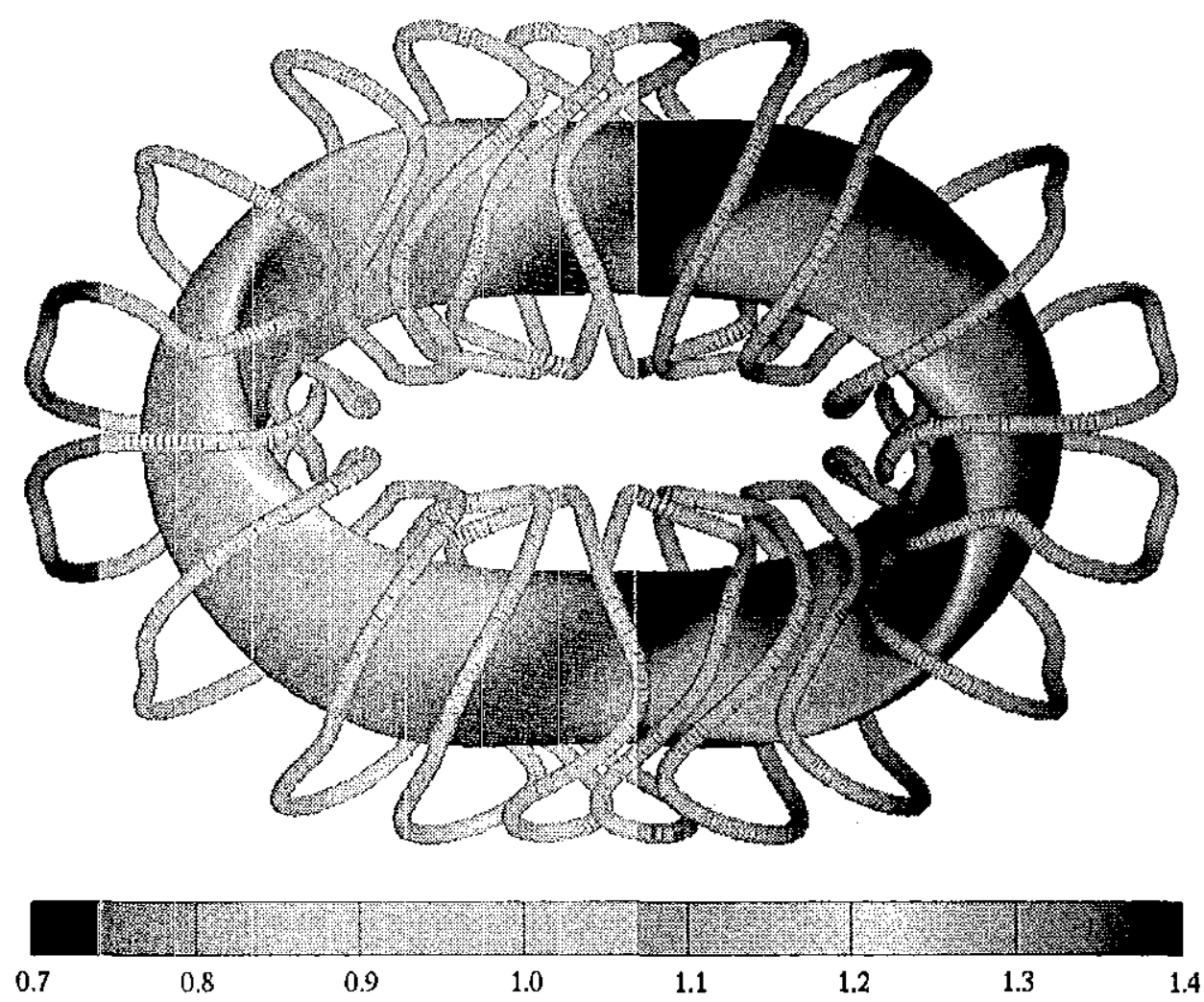

Top view of the QPS plasma and coil configuration. The colors indicate contours of $|B|$ in $T$.

Work supported by U.S. Dept. of Energy under Contract DE-AC05-00OR22725 with UTBattelle, LLC. 


\section{DESIGN OF THE QPS EXPERIMENT}

\section{J.F. Lyon, Oak Ridge National Laboratory, representing the QPS team}

The proposed Quasi-Poloidal Stellarator, QPS, is a low-aspect-ratio $(\mathrm{R} / \mathrm{a}=2.7)$, compact stellarator with a near-poloidally-symmetric magnetic configuration. The QPS parameters are $<\mathrm{R}>=0.9 \mathrm{~m},\langle\mathrm{a}\rangle=0.33 \mathrm{~m},\left\langle\mathrm{~B}_{\mathrm{axis}}>=1 \mathrm{~T}\right.$ for a $1-\mathrm{s}$ pulse, with 1.2-MW ECH and 3-MW ICRF for plasma heating. The stellarator core consists of a modular coil set that provides the primary magnetic field configuration, auxiliary coils including vertical field and toroidal field coils and an ohmic heating solenoid, machine structure, and an external bell-jar vacuum vessel. The modular set has two field periods with eight modular coils per period. Due to symmetry, there are only four different coil types. Flexible copper cable conductor would be wound on a form, vacuum impregnated with epoxy, and canned for compatibility with the vacuum. The coil form also allows the coils to be connected into an integral structure. There is excellent access inside the vacuum vessel for plasma diagnostics and heating. Four years would be required from start of design to first plasma. The status of the engineering design and analysis is described.

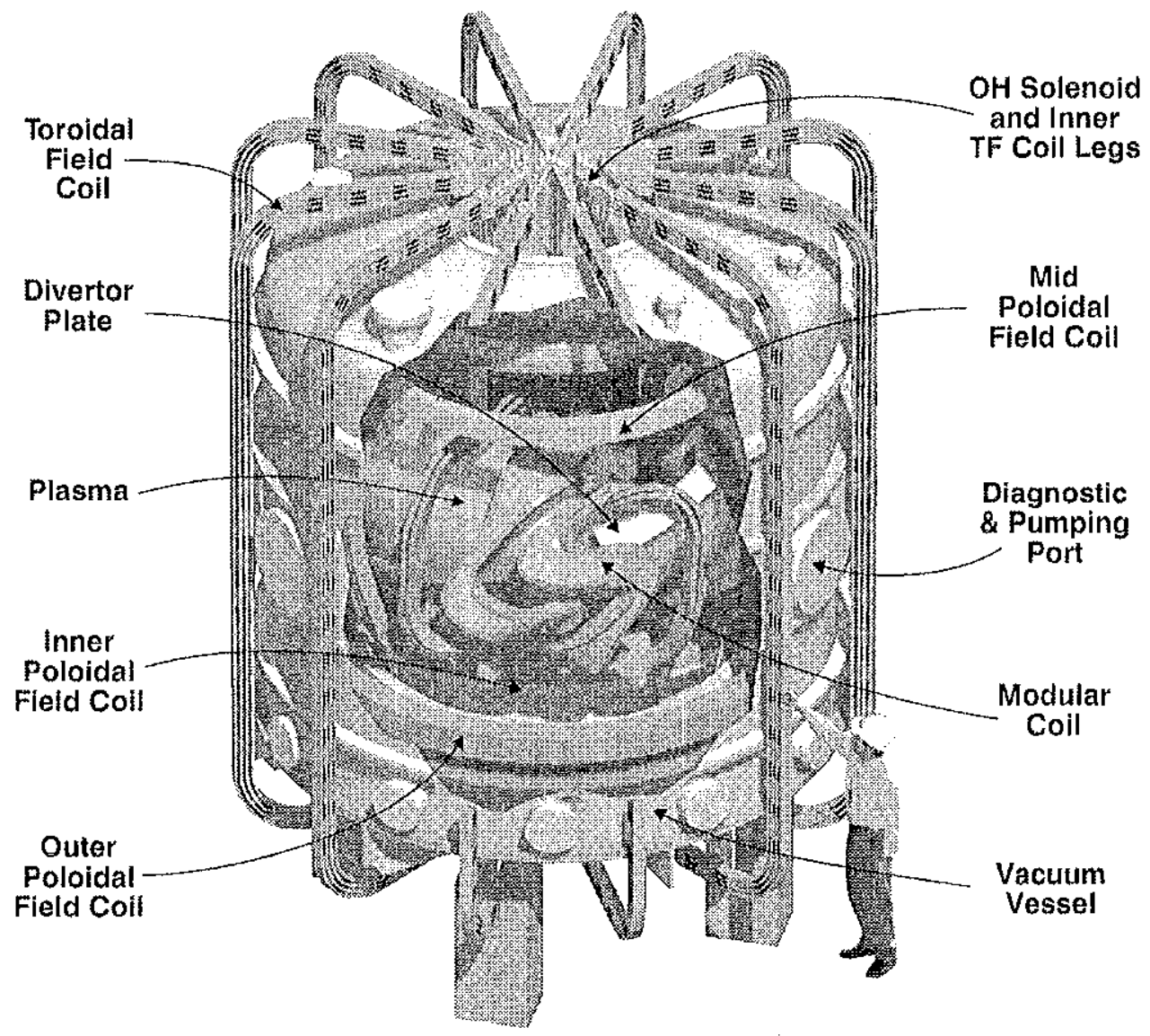

Cutaway view of QPS in its bell jar vacuum tank.

Work supported by U.S. Dept. of Energy under Contract DE-AC05-00OR22725 with UTBattelle, LLC. 
A Lithium Wall Tokamak Experiment* - R. Majeski, R. Kaita, and L. Zakharov, Princeton Plasma Physics Laboratory -- Experiments have been performed on the Current Drive experiment - Upgrade (CDX-U) at the Princeton Plasma Physics Laboratory to investigate the suitability of the use of liquid lithium as a plasma facing component, as part of the ALPS Fusion Technology program. These experiments have largely confirmed the expected low recycling properties of liquid lithium. Theory and modeling [1] indicate that a tokamak discharge entirely bounded by an absorbing, nonrecycling wall will have novel, very favorable properties for confinement and stability. Discharges with peaked electron density, and flat temperature and current density profiles, should result. If the temperature of the lithium layer is maintained at a relatively low value (less than $400^{\circ} \mathrm{C}$ ) by the heat capacity of the wall (in a small experiment) or by fast flow of liquid lithium (in a reactor), then the lithium covered wall can be situated right at the plasma boundary. The wall will thus perform three functions: particle control, energy extraction, and stabilization of the plasma by the wall. The end result would be a tokamak discharge which is stable at far higher beta values than could be accessed in a discharge with conventional peaked current and temperature profiles. Factor-of-ten increases in the beta limit for a TFTR-like device employing close fitting flowing lithium walls have been predicted. Such an innovative regime is of great interest for both plasma science and for fusion applications. Here we, first, present recent results from experiments on CDX-U which characterize plasma behavior with a large-area (2000 $\mathrm{cm}^{2}$ ) liquid lithium limiter. However, at present, wall areas in CDX-U which are not covered with molten lithium still constitute recycling sites. Therefore, we also discuss the possibility of a new test of the lithium wall tokamak concept built around the present CDX-U device. Lithium coatings applied to a heated, conducting wall, installed in the present vessel to completely surround the discharge, would provide the nonrecycling boundary conditions for the plasma. Core fueling would be provided by a multiple pellet injector. The new experiment would be designed to test the prediction of novel, flat temperature and current profiles in a tokamak discharge with a completely nonrecycling wall.

*Supported by US DOE contract \#DE-AC02-76CH-03073

[1] L.E.Zakharov Bull. Am. Phys, Soc. 45, 225 (2000). 


\section{Status of LDX Fabrication}

M. E. Mauel, D. T. Garnier, A. Hansen, T. Sunn Pedersen, E. Ortiz, Columbia University J. Kesner, C. M. Jones, I. Karim, J. Minervini, P. Michael, A. Radovinsky, J. H. Schultz, B. A. Smith, A. Zhukovsky, MIT Plasma Science and Fusion Center

The Levitated Dipole Experiment (LDX) will be the first experiment able to study high-beta plasma confined by a magnetic dipole with near classical energy confinement. LDX consists of three superconducting magnets and illustrates the role of innovative magnetic technology that makes possible explorations of entirely new confinement concepts. In this poster, we describe the LDX machine design and detail the fabrication status of the superconducting floating-coil, charging-coil, and levitation-coil. In addition, we summarize our plan to test the operation of the superconducting magnets and our procedure to cool, to inductively charge, and to levitate the 1.3 MA floating coil.

The LDX Team is a partnership of plasma scientists and magnet technology experts. In order to achieve our scientific objectives, LDX required innovative engineering and design. The LDX design was motivated by several factors: (1) a sufficiently large size to allow for large magnetic flux expansion, (2) the highest possible field consistent with low-weight which allowed use of ECRH to start-up high-beta plasma equilibria $(\beta \sim 30 \%)$ and reduced effects from field-errors and from levitation fields, (3) the experience from previous levitron and spherator experiments, and (4) desire for simple cyrogenics. The resulting design consisted of three innovative magnets: (1) a high-field persistent $\mathrm{Nb}_{3} \mathrm{Sn}$ floating coil with low mass, small size, a light-weight cyrostat with distributed supports having low-thermal conductivity, and relatively easy-to-operate cyrogenics, (2) a large, inductive charging system designed for thousands of high-field cycling through daily charging and discharging, and (3) fusion's first high-temperature superconducting magnet providing a distant

levitation coil coupled to a laser-based stabilization system.

All three coil systems will be completed during the next few months. The floating Coil (714 turns, $\mathrm{Nb}_{3} \mathrm{Sn}, 5 \mathrm{~T}, 1.3 \mathrm{MA}$-turns) has been completed and tested at full current. Ability Engineering (South Holland, IL) is completing final assembly of the outer cryostat. The charging coil (8000 turns, NbTi, 5T, 4.2 MAturns) has been wound by SINTEZ/Science Technology Center (St. Petersburg, Russia), and manufacturing of its cyrostat has begun. Finally, the $\mathrm{HT}_{\mathrm{c}} \mathrm{SC}$ levitation Coil (1310 turns, steelreinforced $\mathrm{Bi}-2223,0.275 \mathrm{MA}-t$ urns) is being built by Everson Electric (Allentown, PA). Winding of this coil should begin by this meeting.

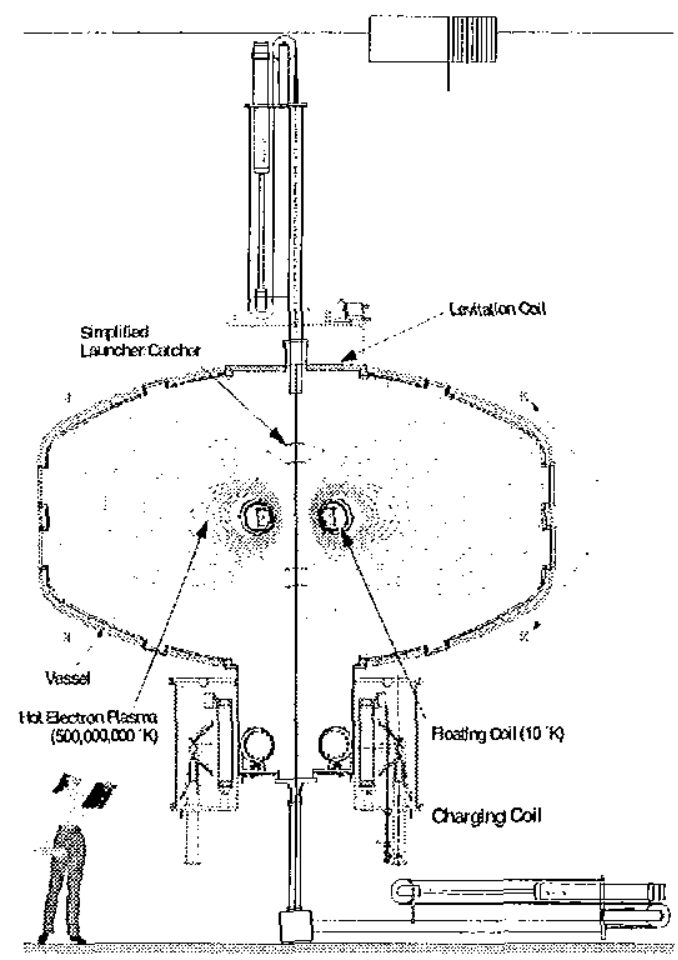




\title{
Oscillating Field Current Drive in the Madison Symmetric Torus
}

\author{
K.J. McCollam, A.P. Blair, J.S. Sarff, J.K. Anderson, D. Craig, \\ W.X. Ding, F. Ebrahimi, G. Fiksel, C.B. Forest, P.D. Nonn, \\ S.C. Prager, S.D. Terry, J.C. Wright, University of Wisconsin
}

Oscillating field current drive (OFCD), or 'F-Theta Pumping', is tested in the Madison Symmetric Torus (MST). In OFCD, an AC toroidal voltage is applied to the plasma 90 degrees out of phase with an applied AC poloidal voltage. This results in net magnetic helicity injection, and OFCD is hoped to be a steady-state current drive method for the reversed field pinch (RFP). The first experimental results for OFCD in MST are reported, which show the current drive effect. Also shown are the effects of OFCD on RFP magnetic fluctuations, plasma energy content, and plasma-wall interaction. An experimental study of AC magnetic field penetration into the RFP is shown. Here it is found, for instance, that the sawtooth oscillation cycle in MST is entrained to the applied AC voltage cycle, and that the sawtooth aids field penetration to the RFP core. Also, the applied AC poloidal voltage seems to affect ion heating and mode dynamics in RFP transport. The experimental results for OFCD are compared to theoretical calculations, and these agree with each other in basic respects. 


\title{
Abstract
}

Innovative Confinement Concepts - ICC2002

\section{Initial Exploration of the Potential for Driving TN Fusion Using Sonic Cavitation}

\author{
William C. Mead \\ woniounst.com \\ Adaptive Network Solutions Research, Inc. \\ Los Alamos, NM \\ D. F. Gaitan, and R. Tessien \\ gaitan@impulsedevices.com, ressien@impulsedevices.com \\ Impulse Devices, Inc. \\ Grass Valley, CA
}

Sonoluminescence (SL) has received much attention in recent years as a fascinating physical phenomenon. ${ }^{1}$ Hydrodynamic modeling has been able to account for many aspects of the bubble dynamics. ${ }^{2}$ We have begun to explore the possibility that sonic cavitation reactors could be designed to drive fusion. We discuss key physical processes and present calculations performed using the HYADES 1-D hydrodynamics code. ${ }^{3}$ Our HYADES calculations have been built on the foundation of a number of hydrodynamics test cases and a representative sonoluminescent bubble example, discussed in a separate report. ${ }^{4}$ We describe intriguing results of preliminary simulations indicating that achieving detectable fusion yields in the laboratory might be feasible. ${ }^{5}$ We present a few highlights of recent work exploring the calculated scaling of bubble performance with key parameters such as bubble mass. ${ }^{6}$

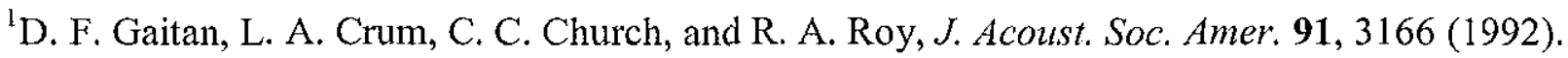

${ }^{2}$ W. C. Moss, D. B. Clarke, J. W. White, and D. A. Young, Phys. Fluids 6, 2979 (1994).

${ }^{3} J$. T. Larsen and S. M. Lane, JQSRT 51, 179 (1994).

4"Hydrodynamic Simulations for a Prototypical Sonoluminescent Air-Bubble-in-Water Example," W. C. Mead, Adaptive Network Solutions Research Rpt. SL_Ex1 (July, 2000).

5“"Preliminary Design Calculations for an LiD/D2 Sonically Driven Reactor Core to Produce Detectable Fusion Reactions," W. C. Mead, Adaptive Network Solutions Research, Inc. Rpt. ANSR_SF_00 (October, 2000).

"'Sonic Cavitation-Driven Fusion: Calculational Exploration of Bubble Performance Scaling with Some Key Design Parameters," W. C. Mead, Adaptive Network Solutions Research, Inc. Rpt. ANSR_SF_02a (January, 2002). 


\title{
A Comparison of Numerical Simulations and Experimental Probe Measurements in RMF Driven FRCs
}

\author{
Richard D. Milroy and Kenneth E. Miller \\ Redmond Plasma Physics Laboratory, University of Washington
}

It has been found that RMF penetration will not occur until the drive parameter $\gamma=\omega_{c c} / \nu_{e j}$ exceeds the penetration parameter $\lambda=r_{s} / \delta$, where $r_{s}$ is the separatrix radius and $\delta=\left(2 \eta / \mu_{o} \omega\right)^{1 / 2}$ is the classical skin depth. When this condition is satisfied, the RMF is capable of exerting a torque sufficient to overcome the resistive drag on electrons that are co-rotating with the RMF, and full penetration can occur. However, experimentally it was found that when RMF is used in conjunction with flux conserving boundaries to drive current in an FRC, full penetration is not observed. Instead, the RMF only penetrates to the magnetic field null, so there is no direct RMF current drive on the inner field lines. A 2-D $(r-\theta)$ Hall MHD model was developed to model this process, and it was found that this phenomenon could be explained with a radially inward plasma flow (which must also be accompanied by a phenomenologically implemented axial flow). Then the $u_{r} \times B_{z}$ component of Ohm's law can provide the needed $E_{\theta}$ on the inner field lines. We think the ability to experimentally realize quasi-steady state flux maintenance is related to maintaining this radial flow. Even though we have only used a simple $r-\theta$ model, it correctly predicts the same partial penetration feature of the RMF, and the $B_{z}$ profile has the same distinct flattening in the region near the null as observed experimentally. We will present a detailed comparison of magnetic probe measurements with the numerical predictions. 


\section{Engineering Design of the National Compact Stellarator Experiment (NCSX)}

G. H. Neilson ${ }^{1}$, W. T. Reiersen ${ }^{1}$, B. E. Nelson ${ }^{2}$, A. Brooks ${ }^{1}$, T. Brown ${ }^{1}$, M. J. Cole ${ }^{2}$, F. Dahlgren', H. M. Fan', P. J. Fogarty ${ }^{2}$, P. Goranson', P. Heitzenroeder', G. Jones ${ }^{2}$, C-H. Jun ${ }^{1}$, D. E. Williamson ${ }^{2}$

I Princeton Plasma Physics Laboratory, Princeton, NJ 08543-0451

${ }^{2}$ Oak Ridge National Laboratory, Oak Ridge, TN 37831-8071

The engineering design of the National Compact Stellarator Experiment (NCSX) is being developed to meet requirements for a proof-of-principle facility to develop the physics of compact stellarators. The core device is a medium-scale $(1.4 \mathrm{~m})$, low aspect ratio (4.3) stellarator whose rotational transform is generated by a combination of internal $(25 \%$ at the edge) and external (75\%) sources. It is optimized around a 3-period reference quasiaxisymmetric plasma that is stable at beta $=4 \%$. It provides substantial flexibility in configuration properties (e.g., internal and external transform, shape, ripple) that affect the physics so that physics models can be tested. The device has a field on axis of up to $2 \mathrm{~T}$ and a nominal plasma current of $175 \mathrm{kA}$. Co- and counter-injected neutral beams will be used to heat the plasma. Provisions will be made to accommodate RF heating as an upgrade. An extensive complement of diagnostics can be accommodated. The engineering design of the stellarator core has evolved to address these needs and requirements. A magnet system consisting of 18 modular coils of three different shapes, with additional toroidal field (TF) and poloidal field (PF) coils controls the magnetic configuration. A conformal inconel vacuum vessel is used. A stand-alone liner composed of formed carbon fiber composite (CFC) panels will provide power and particle handling. The liner accommodates local pumped divertors and is bakeable to $350 \mathrm{C}$. Key features of the design are described in detail. The rationale for the design choices is presented.

* This work was supported by United States Department of Energy Contract No. DE-AC0276-CHO-3073. 


\title{
Fast Particle Confinement Studies on MST using HXR flux and Fokker-Planck Modelling
}

\author{
O’Connell R, Den Hartog DJ, Forest CB, Anderson JK, \\ Biewer T, Fiksel G, Harvey RW, Prager SC, Reardon JC, Sarff JS, Terry SD, \\ University of Wisconsin-Madison
}

Measurements of the fast particle confinement in improved confinement MST dicharges suggest well defined flux surfaces in the core and low fast particle diffusion. The hard X-ray flux spectrum $(10-200 \mathrm{KeV})$ has been measured using a $\mathrm{CdZnTe}$ detector with up to $1 \mathrm{~ms}$ time resolution and $\approx 5 \mathrm{KeV}$ energy resolution during standard and improved confinement plasmas. The measured spectra give direct evidence for the existence of well defined flux surfaces with connection lengths $\left(L_{c}>10^{4} \mathrm{~m}\right)$. Simulations of electron transport during improved confinement plasmas using the Fokker-Planck code CQL3D suggest that diffusion of fast electrons is lower than Rechester-Rosenbluth scaling $\left(<<v_{t h} B_{r}^{2}\right)$ since the simulations require a diffusion coefficient independent of thermal velocity to predict the measured hard $\mathrm{X}$-ray flux. The CQL3D simulations also give estimates for particle, current and energy densities of the fast electron population: initial simulations show densities up to $2 \%$ and current density fractions up to $30 \%$ influencing power calculations and current drive. The calculated parallel electric field is consistent with power balance and equilibrium measurements. 
ICC2002 Abstract

\title{
Experimental Studies of Space Charge Dominated Beams for Heavy Ion Inertial Fusion (HIF)
}

\author{
P.G. O'Shea, S. Bernal, Y. Cui, D. Feldman, M. Glanzer, T. Godlove, J. Harris, H. Li, \\ R.A. Kishek, B. Quinn, M. Reiser, A. Valfells, M. Virgo, M. Walter, M. Wilson, R. Yun \\ Institute for Research in Electronics and Applied Physics, University of Maryland, \\ College Park, MD 20742 \\ I. Haber, \\ Naval Research Laboratory, Washington DC.
}

A detailed understanding of the physics of space-charge dominated beams is vital in the design of heavy ion inertial fusion (HIF) drivers. In that regard, low-energy, highintensity electron beams provide an excellent model system. The University of Maryland Electron ring (UMER), currently under construction, has been designed to study the physics of space-charge dominated beams with extreme intensity in a strong focusing lattice with dispersion. The tune shift in UMER will be more than an order of magnitude greater than in exiting synchrotrons and rings. The $10-\mathrm{keV}, 100 \mathrm{~mA}$, UMER beam has a generalized perveance in the range of 0.0015 , and a tune depression of 0.12 . Though compact (11-m in circumference), UMER is a very complex device, with over 140 focusing and bending magnets. We report on the unique design features of this research facility, the beam physics to be investigated, simulation studies and first experimental results.

\footnotetext{
${ }^{*}$ Research supported by the US Department of Energy.
} 


\title{
Inertial Fusion Energy with the Advanced Fusion Fuels
}

\author{
L. J. Perkins, M. Tabak, B. G. Logan \\ Lawrence Livermore National Laboratory \\ Y. A. Omelchenko, D. E. Baldwin \\ General Atomics, San Diego, CA
}

\begin{abstract}
We are exploring the potential of IFE targets based on $D+D$ or $D+{ }^{3} H e . A$ major advantage of these "advanced" fuels over conventional D-T is that, with appropriate design, most $(\geq 90 \%)$ of the fusion energy escapes the target as energetic charged particles and not as fast neutrons. This suggests the potential for:
\end{abstract}

(a) Coupling charged particle energy directly to advanced, high-efficiency energy conversion schemes thus bypassing the need for complex and expensive conventional thermal conversion

(b) Use of directed charged particle thrust for advanced space propulsion for deepspace missions.

We will present ICF target designs based on $\mathrm{D}-{ }^{3} \mathrm{He}$ main fuel and, by means of "spectrum convertors" surrounding the hohlraum, seek to optimize the charge particle output and its spectral characteristics. Attention will also be directed to interaction of the exit charged particle stream with a cusp magnetic guide field in the chamber and initial results from a hybrid PIC code (particle ion, fluid electron) are presented .

Of course the reactivities of the D-D and D- ${ }^{3} \mathrm{He}$ reactions only become comparable to that of D-T at significantly higher plasma temperatures. Thus such targets will require typical areal densities of $\geq 10 \mathrm{~g} / \mathrm{cm}^{2}$ for adequate fuel burn up and gain. Accordingly, "fast ignition" techniques are required to make these concepts viable at (compression) driver energies in the $\leq 10 \mathrm{MJ}$ range. Adequate performance may be achievable by depositing the fast ignition energy in a small, pre-compressed region of D-T fuel that acts as an ignitor for the main $\mathrm{D}-{ }^{3} \mathrm{He}$ fuel. The overall tritium inventory in the capsule can be less than $1 \%$ and, with appropriate design, these targets can be self-sustaining in tritium through the $\mathrm{D}(\mathrm{d}, \mathrm{p}) \mathrm{T}$ branch of the D-D reaction, thus obviating the need for any tritium breeding in the external plant (S.Atzeni, M. Ciampi, Nucl Fusion, 37 l665 (1997)).

In the design of these targets, a key optimization task is to match the output charged particle kinetic energy spectrum to the external guide field and to the requirements of the energy conversion system or, for advanced space propulsion, the thrust conversion system.

This work was performed under the auspices of the U.S. Department of Energy by the University of California, Lawrence Livermore National Laboratory under contract No. W-7405-Eng-48. 
Doppler Effect Measurement of Ion Spin-up in an FRC with RMF Current Drive. A. M. Peter, E. A. Crawford, University of Washington. The TCS device has been equipped with an intensified CCD spectrometer to determine ion toroidal rotation via the Doppler effect. C-III emission at $229.7 \mathrm{~nm}$ is viewed at six chords, allowing determination of a rotation profile. In the presence of electrons synchronous with the $\mathrm{RMF}$, the ions experience a frictional drag force and will spin up in a short time $\tau=\left(m_{i} / m_{c}\right) v_{\perp}^{-1}$, or $\sim 100 \mu \mathrm{s}$ for TCS. This is not observed in these low density, low temperature experiments; rather, the ions spin up to a small fraction of the electron velocity $\left(3 \%\right.$ at $\omega_{\mathrm{RMF}}=1.0 \mathrm{MHz}, 15 \%$ at $\left.\omega_{\mathrm{RMF}}=0.5 \mathrm{MHz}\right)$ and the current is maintained in steady state, even with $\omega_{c i} / \omega_{R M F}$ as high as 0.65 . The primary braking mechanism may be charge-exchange with a background of cold, non-rotating neutrals. However, the inferred charge-exchange rate requires a neutral density which is higher than that suggested by power balance. There may be additional braking mechanisms, such as shorting of the radial electric field or preferential loss of ions rotating in the paramagnetic direction. The ion rotation usually results in the $n=1$ or $n=2$ rotational instabilities seen in standard theta-pinch formed FRCs. These modes generally saturate at low levels and multipole stabilization has not been necessary. In the future it may be desirable to control the ion rotation using oppositely directed neutral beams. 


\section{Dynamics of strong proton rings in the Cornell Ion Ring Experiment}

W.J. Podulka, J.B. Greenly, A.V. Gretchikha, D. Lee, Cornell

University

In the Cornell ion ring experiment FIREX, a proton ring is formed in one ion cyclotron period by cusp injection into a solenoid. The rapid magnetic field change generated by the ring current launches large-amplitude Alfven waves with amplitudes as large as $25 \%$ of the $6 \mathrm{kG}$ field. In high-density plasmas, the predominant component of the wave spectrum is the lowest-order (nearly radial) magnetoacoustic mode of the magnetized plasma column in the solenoid. In lower density plasmas in which the Alfven speed approaches the ring ion velocity, a much more complex wave spectrum is generated. Significant heating of the background plasma by the Alfven waves and substantial azimuthal energy loss by the ring particles is observed. Diamagnetic loop arrays and magnetically-insulated proton detectors diagnose the wave and ring dynamics, and spectroscopic diagnostics measure energy deposition in the background plasma. We present our devcloping understanding of the ring-plasma dynamics, including ring stability and particle loss, and details of the magnetic dynamics. 


\title{
Coaxial Helicity Injection for plasma start-up in NSTX*
}

\author{
R.Raman ${ }^{1}$, D.Gates ${ }^{2}$, T.R.Jarboe ${ }^{1}$, D.Mueller ${ }^{2}$, B.A.Nelson ${ }^{1}$, M.J.Schaffer ${ }^{3}$, R.Maqueda ${ }^{4}$, \\ J.Menard ${ }^{2}$, M.Nagata ${ }^{5}$, F.Paoletti ${ }^{6}$, S.Paul ${ }^{2}$, S.Sabbagh ${ }^{6}$, C.H.Skinner ${ }^{2}$, V.Soukhanovskii ${ }^{2}$, \\ D.Stutman ${ }^{7}$, X. Tang ${ }^{4}$, L. Zakharov ${ }^{2}$ and the NSTX Research Team \\ ${ }^{\prime}$ University of Washington, Seattle, WA 98195 \\ ${ }^{2}$ Princeton Plasma Physics Laboratory, Princeton, $N J$ \\ ${ }^{3}$ General Atomics, San Diego, $C A$ \\ ${ }^{4}$ Los Alamos National Laboratory, Los Alamos, NM \\ ${ }^{5}$ Himeji Institute of Technology, Himeji, Japan \\ ${ }^{6}$ Columbia University, New York, $N Y$ \\ ${ }^{7}$ Johns Hopkins University, Baltimore, $M D$
}

To minimize the aspect ratio of toroidal plasmas, elimination of the central solenoid is a consideration for future ST designs. Implementation of CHI on NSTX draws on extensive experience from the HIT experiments at the University of Washington. Experiments on NSTX thus far have succeeded in attaining 390kA of CHI generated toroidal current using about $28 \mathrm{kA}$ of injector current. $\mathrm{CHI}$ discharges have been obtained without relying on the ohmic solenoid. Methods to safely ramp the CHI produced toroidal current to durations over $330 \mathrm{~ms}$ have been developed. Discharges obtained on NSTX are more than an order of magnitude longer than previously attained by $\mathrm{CHI}$ in a spheromak device or an ST. A rotating $n=1$ mode, previously observed in optimized discharges on smaller STs driven by CHI, has been observed for the first time in NSTX CHI discharges. The flux utilization efficiency continues to be high, approaching 100\%. EFIT and TSC codes are being used to assess flux closure.

Experiments to date have shown that $\mathrm{CHI}$ engineering systems can be applied to a large ST for the production of substantial toroidal current. Our next step plans on NSTX are to produce a high current long flat-top discharge for characterization with and without auxiliary heating, to implement feedback control algorithms, and to modify the absorber region. Absorber modifications will allow for the implementation of two feedback control systems. An absorber feedback control system (based on the HIT-II system) to reduce unwanted magnetic fields in the absorber and an equilibrium feedback control system, (based on the present NSTX flux expansion method) to predict and correct the boundary flux. Feedback control of NSTX discharges should allow improved control of the discharge and enable one to retain the large amount of closed flux that is expected from future $500 \mathrm{kA}$ CHI discharges on NSTX.

*Work supported by US DOE contract numbers: DE-AC02-76CH03073 and DE-AC05-00R22725. 


\title{
Current Drive Experiments in the HIT-II Spherical Torus
}

\author{
A. J. Redd, T. R. Jarboe, R. G. O'Neill, P. Gu, W. T. Hamp, \\ V. A. Izzo, B. A. Nelson, R. Raman, P. E. Sieck and R. J. Smith, \\ Aerospace and Energetics Research, University of Washington, \\ Box 352250, Seattle, Washington 98155-2250
}

The Helicity Injected Torus [HIT-II: T. Jarboe, et al, Phys. Plasmas 2, 1807 (1998)] is a low-aspect-ratio tokamak capable of both inductive (Ohmic) and Coaxial Helicity Injection (CHI) current drive. HIT-II is modest in size (major radius $R=0.3 \mathrm{~m}$, minor radius $a=0.2 \mathrm{~m}$ and on-axis toroidal field of up to $0.5 \mathrm{~T}$ ), but has.demonstrated $200 \mathrm{kA}$ of toroidal plasma current, using either $\mathrm{CHI}$ or induction separately. The loop voltage, boundary flux and plasma equilibrium are controlled by a real-time flux feedback system. HIT-II Ohmic plasmas exhibit reconnection events during both the current ramp-up and decay, events which relax the current profile while conserving the magnetic helicity. A new operating regime for $\mathrm{CHI}$ plasmas, using a double-null divertor (DND) boundary flux, has been explored. DND CHI plasmas exhibit good shot-to-shot reproducibility, low impurity content, minimal shorting current in the absorber region, and EFIT-reconstructed equilibria consistent with significant closedflux core regions [EFIT: L. Lao, et al., Nucl. Fusion 25, 1611 (1985)]. HIT-II DND $\mathrm{CHI}$ discharges also exhibit a continuous $n=1$ mode at the outer midplane, a mode which has been correlated experimentally with current-profile relaxation. A detailed explanation of helicity injection current drive has been developed which is consistent with experimental observations of HIT and HIT-II discharges. According to this mechanism, asymmetric distortion of the $n=1$ mode structure generates current drive in the core plasma by dynamo action, relaxing the CHI-driven current profile. This relaxation can also generate differential rotation between the core plasma and the driven edge region, a result that is observed experimentally. 


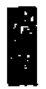

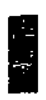

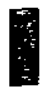

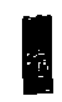

I

:-

3

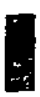

$\therefore$

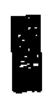

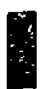

$\therefore$

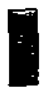

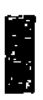




\title{
Application of the Penning trap to the study of neoclassical transport
}

\author{
S.H. Robertson, Q. Quraishi, R.A. Walch, Center for Integrated \\ Plasma Studies
}

In a Penning trap confining electrons there is diffusion and mobility from collisions of the elcctrons with background gas, as well as some anomalous transport arising from asymmetric fields. A conductor along the axis of the Penning trap has been used to create an azimuthal field which results in banana-like electron orbits. Experimental studies have shown a mobility drift from a radial electric field and a drift arising from an azimuthal (ohmic heating) electric field (Ware's drift) $[1,2]$. The measured transport has the neoclassical scaling: inversely with the square of the axial field and no dependence upon the azimuthal field. Experiments are in progress to extend the parameter range in order to observe the transition regime from classical transport to neoclassical that occurs when the collision frequency becomes greater than the axial bounce frequency.

[1] S. Robertson, J. Espejo, J. Kline, Q. Quraishi, M. Triplett and B. Walch, Physics of Plasmas 8, 1863-1869 (2001).

[2] J. Kline, S. Robertson, N.I. Triplett, and B. Walch, Physical Review E 63, 056406 (2001). 


\section{Active experiments on determining the quality of magnetic flux surfaces in a spheromak}

\section{D.D. Ryutov, Lawrence Livermore National Laboratory}

There are reasons to believe that, at some stages of spheromak discharges, especially during the so-called decay phase, relatively good magnetic flux surfaces can be formed. We suggest to study an effect of a calibrated external perturbation of the magnetic field on the confinement properties of a spheromak (and, specifically, of the SSPX spheromak at Livermore) and thereby obtain an information on the quality of the flux surfaces. One can expect that, by a gradual increase of a non-axisymmetric perturbation, one can reach formation of the islands and their eventual overlapping. This would show up in significant degradation of plasma parameters. Another signature of a disappearance of good flux surfaces would be a disappearance of runaway electrons. A gross change of the plasma equilibrium will affect also signals from the magnetic probes. We consider several ways of introducing these perturbations: by an external coil, by an external permanent magnet, and by an internal permanent magnet. We describe the island formation in a model of a "rectified" spheromak with a non-circular cross-section and evaluate the magnitude of perturbations required to reach various degrees of deformation of the flux surfaces. We find that equilibria with flat distribution of the safety factor are most vulnerable to the external perturbations. We also note that the introduction of nonaxisymmetric perturbations in the situation, where magnetic field lines begin and end on the electrodes (which is supposedly the case during the formation phase), has only a relatively minor effect on the plasma parameters.

This work was performed under the auspices of the U.S. Department of Energy by University of California Lawrence Livermore National Laboratory under contract W-7405-Eng-48. 


\title{
Drift instabilities in a strongly collisional, $\beta>1$ plasma
}

\author{
D.D. Ryutov \\ Lawrence Livermore National Laboratory, Livermore, CA 94551, USA
}

Some versions of Magnetized Target Fusion devices will be using a very high beta plasma, with local beta in the range of 10-20 and more. Drift instabilities in a very high beta plasma are electromagnetic and are quite different from the analogous instabilities in a low beta plasma. In a collisionless limit they have been analyzed by El Nadi and Rosenbluth ("Infinite $\beta$ - limit of the drift instability," Phys. Fluids, 16, 2036, 1973) who have shown that the cross-field transport coefficients in such a plasma reach a Bohm value. On the other hand, high-density plasma in MTF systems is usually strongly collisional in the sense that the drift frequency $\omega_{D \sim}(c T / e B a) k$ (with $a$ being the plasma radial length-scale, and $k$ being the wave number) for large-scale perturbations $\left(k \rho_{i}<<1\right)$ is smaller than the ion-ion collision frequency. This regime is studied in the present paper. We show that plasma becomes generally more stable, and transport coefficients fall well below the Bohm level. We address also a special version of the drift instability caused by the presence of high- $Z$ impurities. Its collisionless analog has been treated by Coppi, Furth, Rosenbluth, and Sagdeev (Phys. Rev. Lett. , 17, 377, 1966). We demonstrate that this instability may also be weakened in a strongly collisional plasma. This work was performed under the auspices of the U.S. Department of Energy by University of California Lawrence Livermore National Laboratory under contract W7405-Eng-48. 


\title{
Improved Confinement and Future Plans in MST
}

\author{
J.S. Sarff, J.K. Anderson, T.M. Biewer, S. Castilo, B.E. Chapman, D. Craig, D.J. Den Hartog, G. \\ Fiksel, C.B. Forest, R. O'Connell, S.C. Prager, J.C. Reardon \\ University of Wisconsin-Madison \\ D.L. Brower, W.X. Ding, S. Terry \\ University of California-Los Angeles \\ K.A. Connor, D.R. Demers, P.M. Schoch \\ Rensselaer Polytechnic Institute
}

Control of magnetic turbulent transport in the reversed field pinch yields increased energy confinement, plasma temperature, and beta. Relative to standard toroidal induction with incumbent dynamo relaxation, added poloidal current drive in MST increases the energy confinement time nine-fold to $9 \mathrm{~ms}$, increases beta from $9 \%$ to $14 \%$, and permits electrons to exceed $1 \mathrm{keV}$ despite decreased Ohmic heating, a clear demonstration of reduced transport. The electron heat diffusivity drops to $\sim 5 \mathrm{~m}^{2} / \mathrm{s}$, comparable to typical tokamak plasma values. Fast electrons up to $100 \mathrm{keV}$ are confined, suggesting a transition to well-formed magnetic surfaces. An increased understanding of the magnetic transport process and the role of the magnetic spectrum is resulting, supported by new diagnostic capabilities. Core magnetic fluctuations are detected with FIR polarimetry, the core mean magnetic field is measured by MSE, the ion temperature is measured by CHERS and Rutherford scattering, and the electric field by heavy ion beam probing. New auxiliary systems are in development: neutral beam heating to investigate beta limits, rf current drive (lower hybrid and EBW) to refine current profile control, and oscillating field current drive to test Ohmic dc current sustainment.

Work supported by US DoE 


\title{
ADVANCES IN NOVEL FRC PLASMAS ${ }^{*}$
}

\author{
M.J. Schaffer, M.R. Brown, $†$ J.A. Leuer, P.B. Parks, C.D. Cothran $\dagger$ \\ General Atomics, P.O. Box 85608, San Diego, California 92186-5608 \\ 'Swarthmore College, Swarthmore, Pennsylvania 19081-1397
}

Field Reversed Configurations (FRCs) can be sustained in steady state by rotating magnetic field current drive, which also greatly improves plasma confinement. ${ }^{1}$ If radial transport is diffusive, near-spherical geometry yields the lowest power steady-state FRC fusion reactors. Furthermore, oblate FRCs can be tilt stabilized by an external wall or active feedback coils. Therefore, short configurations are preferred for steady state FRC fusion reactors. The doublet $\mathrm{CT}$ is a new FRC. Even with no toroidal field $\left(\mathrm{B}_{\mathrm{T}}=0\right)$ it can be interchange-stable inside and outside its internal figure-eight separatrix (by compressibility and average good curvature, respectively) and near its bounding separatrix (by compressibility).

The recent prediction ${ }^{2}$ of FRC tilt stabilization by weak, spontaneously generated toroidal magnetic fluxes leads us to investigate this effect. The Swarthmore Spheromak Experiment $(\mathrm{SSX})^{3}$ has been modified to produce FRCs within a copper cylindrical flux conserver by merging two gun-generated, counter-helicity spheromaks. Frozen flux from external reconnection control coils ( $\mathrm{RCCs}$ ) will control the extent of spheromak reconnection and thus the residual $\mathrm{B}_{\mathrm{T}}$ surrounding the two magnetic axes of a CT doublet equilibrium, to test aspects of the Ref. 2 stability prediction. Single-axis FRCs will also be made and studied.

A new finite-element, Grad-Shafranov, free-plasma-boundary CT equilibrium solver was written and used to design the SSX RCCs, whose frozen flux controls the final plasma shape. The code calculates both doublet and conventional $\mathrm{CT}$ equilibria. It was validated against analytic equilibria, including a new analytic FRC solution.

Two-fluid (electron and single ion species) calculations ${ }^{2}$ show that rotating FRCs self generate $\mathrm{B}_{\mathrm{T}}$. Centrifugal density stratification makes $\nabla \mathrm{p}$ non-perpendicular to magnetic surfaces, especially near their axial extremes, which yields a non-zero $\nabla \times \mathrm{E}$. Total generated toroidal flux is zero, but calculated local $\mathrm{dB}_{\mathrm{T}} / \mathrm{dt}$ are sizeable. Therefore, it might not be necessary to externally drive $\mathrm{B}_{\mathrm{T}}$ if local $\mathrm{B}_{\mathrm{T}}$ is needed for stability.

We outline a D- ${ }^{3} \mathrm{He}$ reactor based on a steady-state oblate FRC.

\footnotetext{
*Work supported by General Atomics IR\&D funds and Grant DE-FG02-00ER54604.

IJ.T. Slough et al., Phys. Rev. Lett. 85 (2000) 1444.

2 Yu.A. Omelchenko, Pliys. Plasmas 7, 1443 (2000).

${ }^{3}$ C.G.R. Geddes, T.W. Kornack, M.R. Brown, Phys. Plasmas 5, 1027 (1998).
} 


\title{
STEADY-STATE, OBLATE, D-3 He FRC FUSION FOR TERRESTRIAL POWER AND SPACE PROPULSION ${ }^{*}$
}

\author{
M.J. Schaffer \\ General Atomics, P.O. Box 85608, San Diego, California 92186-5608
}

This paper presents preliminary concepts for steady-state, D- ${ }^{3} \mathrm{He}$ Field Reversed Configurations (FRCs) for terrestrial fusion power and space propulsion. Basic parameters are scoped for D- ${ }^{3} \mathrm{He}$ FRC fusion power and reactor size and mass. A more or less spherical geometry FRC yields the minimum size for steady state operation. Moreover, oblate FRCs can be tilt stabilized by an external wall or by active feedback. Point designs are given for a terrestrial power reactor and a fusion rocket fusion power core. The specific power is found to be high, justifying further research into this concept.

FRCs are among the most attractive magnetic confinement systems for controlled fusion power. They intrinsically have $\beta \approx 1$, which makes efficient use of the magnetic field. Magnetic field in the hot plasma core is small, virtually eliminating synchrotron radiation loss and opening the way to the high temperatures required by low-neutron fuels. Plasma exhausts naturally to the external world. In principle, exhaust plasma can be used directly for rocket thrust. Exhaust can also be directly converted to make reactor recirculating power and power for sale. Steady state allows superconducting magnets and reduces recirculating power. Steady loads also relieve components from fatigue damage.

To capitalize on its advantages, FRC stability must be guaranteed, and for steady state applications, confinement must become comparable with (suitably scaled) tokamaks. FRCs can be sustained in steady state by rotating magnetic field current drive, which also greatly improves plasma confinement. ${ }^{1}$ Weak internal toroidal magnetic fluxes, which may be spontaneously generated, aid tilt stability. ${ }^{2}$ Fundamental investigation of FRC stability and confinement is the principal task required to validate the steady-state FRC fusion concept.

\footnotetext{
"Work supported by General Atomics IR\&D funds.

1J.T. Slough et al., Phys. Rev. Lett. 85 (2000) 1444.

${ }^{2}$ Yu.A. Omelchenko, Phys. Plasmas 7, 1443 (2000).
} 


\section{Ion Resonance Instability in the Virtual Cathode of the Penning Fusion Experiment}

M.M. Schauer, Los Alamos National Laboratory

The goal of the Penning Fusion eXperiment-Ions (PFX-I) is the production of thermonuclear conditions in a Penning trap by means of spatial and/or temporal compression of a high temperature plasma. The present approach involves confinement of positive ions in a virtual cathode produced by a non-thermal, electron plasma held within a Penning trap of modified geometry. In this poster we review data from destructive measurements of the electron energy distribution and ion inventory in our trap. We then present a new, non-destructive electron density diagnostic based on the ion resonance instability. Finally, progress on a system redesign which will allow higher voltage operation, better control of ion density, and more diagnostic access is presented. 


\section{Sawtooth and Edge Plasma Behavior during ICRH Heating in ET*}

L.W. Schmitz, J.-L.Gauvreau, P.-A. Gourdain, D.J. LaFonteese, R.J. Taylor, University of California, Los Angeles, CA 90024.

The Electric Tokamak $\left(\mathrm{R}=5 \mathrm{~m}, \mathrm{a}=1 \mathrm{~m}, \mathrm{~B}_{\mathrm{t}}<0.25 \mathrm{~T}\right)$ has produced Ohmic and H-mode plasma with excellent energy confinement. Second Harmonic ICRH heating so far has been the most promising RF heating regime. Charge exchange measurements indicate ion temperatures up to $600 \mathrm{eV}$ and tail enery up to $2 \mathrm{keV}$. We report $3^{\text {rd }}$ and $4^{\text {th }}$ Harmonic ECE measurements as well as Langmuir and triple probe data confirming electron heating and energetic electron production during ICRH. Under certain conditions we observe a sudden increase of the ECE radiation from the central plasma region about 200 $\mathrm{ms}$ into the RF pulse, indicative of electron heating and/or the formation of a central transport barrier. Sawtooth propagation and suppression during ICRH application is examined by probes and ECE. We also discuss the effects of RF heating on edge potential, density and electron temperature profiles as measured by a 16 element radial Langmuir probe array. Initial measurements of toroidal plasma flow from $\mathrm{H}_{\mathrm{a}}$ and $\mathrm{C}$ III Doppler spectroscopy are presented.

*This work is supported by U.S. DoE grant \# DE-FG03-86-ER53225 


\section{Construction of the Helicity Injected Torus with Steady Inductive Helicity Injection (HIT-SI)}

P.E. Sieck, W.T. Hamp, V.A. Izzo, T.R. Jarboe, B.A. Nelson, J.A. Rogers, University of Washington

HIT-SI is a "bow tie" spheromak designed to implement Steady Inductive Helicity Injection (SIHI). The engineering requirements of SIHI lead to several unique design features, including a multiply connected electrically insulating o-ring seal and a close-fitting passive flux conserver that is electrically insulated from the plasma. Prototype tests have been performed to verify the performance of the o-ring seal and the plasma sprayed zirconia insulation. Mechanical stresses in the flux conserver have been predicted using a finite element model under atmospheric and magnetic loads. NIMROD simulations for decaying spheromak equilibria in the HIT-SI geometry are presented, including a preliminary survey of the dependence of the evolution on the magnetic Lundquist number, S. Progress in the construction and assembly of HIT-SI will be presented. 


\title{
The MTF prospects for low-cost fusion energy development
}

\author{
Richard E. Siemon ${ }^{a}$, Peter J. Turchib, Daniel C. Barnes ${ }^{a}$, James H. Degnan ${ }^{b}$, \\ Paul Parks ${ }^{\mathrm{c}}$, Dmitri D. Ryutov ${ }^{\mathrm{d}}$, Francis Y. Thio ${ }^{\mathrm{e}}$
}

a) Los Alamos National Laboratory, Los Alamos, New Mexico 87545, USA

b) Air Force Research Laboratory, Kirtland AFB, NM 87117-5776, USA

c) General Atomics, San Diego, CA, 92121-1194, USA

d) Lawrence Livermore National Laboratory, Livermore, CA 94550, USA

e) NASA Marshall Space Flight Center, Huntsville, AL 35812, USA

We have argued for some time that Magnetized Target Fusion (MTF) should be explored because it has the potential to resolve one of the major problems with conventional fusion energy research - the high cost of facilities to do experiments and in general develop practical fusion energy $[1,2,3]$. In this paper we refine that argument by examining the costs for facilities that would be needed to do burning-plasma experiments in a generic MTF context. We find semi-quantitatively that MTF should allow fusion energy production without costly facilities within the same generally accepted physical constraints used for conventional magnetic and inertial fusion. The results are mostly independent of detailed plasma physics, and depend instead on the cost of energy and pulsed power, which we estimate based on existing facilities (NIF, Z, and Atlas.) We further compare the generic MTF costs with a nominal steady-state tokamak cost model as well as three specific types of MTF plasma targets. As an aside, we note that MTF burning plasma experiments are needed in addition to "ignition" experiments being proposed in the tokamak context, because MTF is intrinsically a pulsed, high-pressure, batch-burn type of system with quite different physics issues. For example, MTF is not likely to rely upon alpha heating to any significant extent.

1. I.R.Lindemuth et al., Phys. Rev. Letter 75, 1953 (1995).

2. R.P.Drake, et al., Fusion Tech. 30, 310 (1996).

3. R.E.Siemon et al., Proc. $18^{\text {th }}$ IAEA Conf. on plasma physics and controlled nuclear fusion research, Sorrento, Italy (2000) 
Stability of Axisymmetric Plasmas in Closed Line Magnetic Fields

Andrei N. Simakov, Peter J. Catto, R. J. Hastie, and Jesus J. Ramos MIIT Plasma Science and Fusion Center

The stability of axisymmetric plasmas confined by closed line poloidal magnetic fields is investigated. First, the ideal MHD theory is employed to study isotropic pressure plasmas. The energy principle is used to derive an interchange stability condition and an integro-differential ballooning equation for pressure driven Alfvén modes. The results are applied to a point dipole equilibrium to show that it is stable for arbitrary $\beta$. The ideal MLHD treatment is generalized to include sound waves and effects of plasma resistivity on the Alfvén and sound wave stability. It is found that the resistivity destabilizes the ideally stable modes above some critical $\beta$.

MHD fails to describe many important phenomena and assumes collisional orderings which are often not appropriate for LDX. The important case of semi-collisional plasmas is studied kinetically for arbitrary $\beta$. It is assumed that $\omega_{b}>\nu>\omega \sim \omega_{d} \sim$ $\omega_{*}$, where $\omega_{b}, \nu, \omega, \omega_{d}, \omega_{*}$ are the bounce, collision, mode, magnetic and diamagnetic drift frequencies. An integro-differential ballooning equation for Alfvén modes is derived, which reduces to the ideal MIHD ballooning equation when $\omega>\omega_{d}, \omega_{*}$, but also describes a drift frequency entropy mode when $\omega \sim \omega_{d}, \omega_{*}$. Stability of the modes depends on $\eta=d \ln T / d \ln n, d=\omega_{*}(1+\eta) / \omega_{d}$ and $\beta$. Collisional effects, such as ion gyro-relaxation effects, are important for the entropy mode stability and are found capable of destabilizing it.

A. N. Simakov, Doctoral Dissertation, MIT (2001), and references therein.

Work supported by U.S. D.o.E. 


\title{
RMF Driven FRC Fusion Reactor
}

\author{
John Slough \\ University of Washington \\ Redmond Plasma Physics Laboratory \\ Redmond, WA 98052 \\ PH: 425-881-7706 \\ Fax: 425-882-9137 \\ slough@aa.washington.edu
}

There have been several experiments in the past that have formed and sustained a field-reversed configuration (FRC) with a rotating magnetic field (RMF). But it is only recently that the confinement and scaling properties of these plasmas have been assessed. While this work is still ongoing, recent experiments where there has been adequate separation of the FRC from wall contact, the confinement and stability of the plasmas look promising. These RMF driven FRCs display a resistivity comparable to that predicted by past FRC experiments. Enhanced particle confinement with much better confinement than previous FRC scaling has also been observed. Even with a scaling no better than that observed in past FRC experiments, a fusion system based solely on RMF driven currents is envisioned. The fusion system would be compact ( $\mathrm{rp}<0.1 \mathrm{~m}$ ), and would operate with a significant RMF field $(<1 \mathrm{~T})$. The power flow into the FRC during formation would reach several hundred MW and ohmically heat the FRC to fusion conditions in less than $1 \mathrm{~ms}$. Although the RMF field is large compared to that generated in current experiments, the necessary oscillating currents are within the capability of current solid-state switching technology. Details of the final reactor size and power depend on the confinement scaling observed as $\mathrm{RMF}$ plasmas reach more fusion relevant conditions. Various burn scenarios that include plasma translation with a spatially rotating field are also possible. 


\title{
Fast ignition of strongly magnetized plasma
}

\author{
V.I. Sotnikov, M. Angelova, and B.S. Bauer \\ University of Nevada, Reno \\ V.B. Krasovitskii and Yu.A. Volkov \\ Institute of Applied Mathematics, Moscow
}

Fast ignition [1] could be an important complement to conventional inertial confinement fusion, offering increased fusion energy gain, decreased implosion drive energy, decreased symmetry requirements, simplified target design, and new perspectives on fusion burn. Compressed DT would be locally ignited by an intense laser, and then, in turn, would ignite lower-density $\mathrm{D}_{2}$. Magnetizing the fusion fuel could shrink the region heated by the intense laser, reducing the laser energy required for ignition. Alternatively, magnetization of the fusion fuel may be a side effect of inexpensive current-driven-liner (j $x$ B) compression $[2,3]$ and other methods of fuel preparation [4,5]. To understand the heating of magnetized plasma by intense, ultrashort laser pulses, analytic calculations and numerical simulations have been performed. The response of the plasma to the intense laser pulse takes place in two stages. First, plasma electrons are accelerated to relativistic energies, and expelled from the region occupied by the resting ions, by the laser wave field (the longitudinal component of the Lorentz force). This strongly polarizes the plasma and generates a huge electrostatic wake-field wave, with maximum amplitude behind the rear edge of the pulse. This wake field accelerates ions to high energy. The first-stage electron acceleration has been investigated with cold fluid theory, for an O-mode electromagnetic wave propagating in a uniform magnetized plasma. The later ion-acceleration stage (along with the initial electron-acceleration stage) has been examined with two-dimensional particle-in-cell simulations. Cases of laser pulse propagation along and perpendicular to the magnetic field have been investigated. The focus is on plasma and laser parameters that are readily accessible experimentally, i.e., $1 \mathrm{MG}<\mathrm{B}<100 \mathrm{MG}, 10^{18} \mathrm{~W} / \mathrm{cm}^{2}<\mathrm{I}<10^{21} \mathrm{~W} / \mathrm{cm}^{2}$, duration $0.1-10 \mathrm{ps}, \lambda=1.05 \mathrm{~nm}$, etc. This will help guide the development of ultra-intense laser-plasma interaction experiments at the Nevada Terawatt Facility (NTF, at UNR) and elsewhere.

1. M. Tabak et al., Phys. Plasmas 1, 1626 (1994).

2. F. Winterberg, Z. Naturforsch. 54a, 459-464 (1999).

3. S.A. Slutz et al., Sandia National Lab Report SAND98-2269 (1999).

4. F. Winterberg, Z. Naturforsch. 53a, 933-936 (1998).

5. F. Winterberg, Z. Naturforsch. 55a, 909-911 (2000). 


\title{
Alpha Physics Issues in Compact Stellarators
}

\author{
D. A. Spong, ORNL
}

Compact stellarators share many alpha physics issues in common with tokamaks. For example, both devices are susceptible to some level of alpha particle loss during slowing down due to departures from perfect symmetry (e.g., field ripple from a finite number of coils in the tokamak and deviations from quasi-symmetry in the stellarator). The primary impact of these losses is through localized heat loads on the first wall that must be evaluated and designed around. Alpha populations can also destabilize Alfven modes in both tokamaks and stellarators, leading to the possibility of enhanced losses of fast ions. We will discuss and present results from both computational tools that are currently available and those being developed to address these alpha physics issues in compact stellarators. One advantage of stellarators is the substantial design flexibility possible using numerical optimization methods and 3D shaping. As the above alpha physics issues become better understood it is expected that this design flexibility will lead to reactor configurations with improved optimization for these isstes. 


\title{
Simulating RFP and Spheromak Relaxation with Thermal Transport
}

\author{
Carl Sovinec, Fatima Ebrahimi, UW \\ Bruce Cohen, Simon Woodruff LLNL \\ Eric Held, USU
}

Nonlinear simulations of the RFP and spheromak using the zero-beta MHD model have provided fundamental information on the role of three-dimensional current-driven activity in these configurations. Equally significant insight on the role of pressure-driven activity is expected from simulations with finite-beta models. However, since relaxation occurs on time scales that are comparable to energy transport, basic closures are inadequate. Furthermore, realistic treatments have to account for the extreme anisotropy of transport processes with respect to the magnetic field direction. The NIMROD code [http://nimrodteam.org] address the numerically challenging anisotropy by using high-order finite-element representations. Convergence studies show that realistic parallel to perpendicular diffusivity ratios can be achieved with cubic basis functions, even with severe and nonuniform misalignment between the grid and the magnetic field.

The spatial accuracy afforded by the NIMROD algorithm will be applied to thermal transport in relaxing configurations in two ways. We will first use fluid closures with large parallel coefficients. Though this approach does not capture electron streaming effects, it will reproduce the correct dependence with regard to magnetic field fluctuation amplitudes and stochasticity. Resulting pressure profiles will be qualitatively correct, exciting pressure-driven modes where appropriate. The second approach uses a Chapman-Enskog-like closure for electrons that is valid at arbitrary collisionality and captures free-streaming effects [Held, et al. PoP 8 1171, (2001).]. This approach integrates nonlocal temperature information along magnetic field lines to determine the kinetic heat flux at each point in the computational mesh.

Initial simulation work with the fluid closure is being used to determine if centrally peaked temperature profiles can result with open magnetic field lines in the spheromak configuration. We are finding significant heating from compression, a result worthy of further investigation. We have also found that local pressure-driven modes in the RFP transition from resistive to ideal behavior only at conditions well above the Suydam criterion. 
3
8

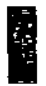

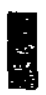

是
-
-

$\therefore$

草

$\therefore$

$\because$

:

$\therefore$

8

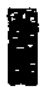


Considerations of Helicity Balance in SSPX

B.W. Stallard, R.H. Bulmer, D.N. Hill, H.S. McLean, S. Woodruff

Lawrence Livermore National Laboratory, Livermore, CA 94550

Using helicity balance to analyze and predict plasma performance is challenging.

Relevant parameters for helicity balance are gun voltage, including wall sheaths, gun flux captured during spheromak formation, and helicity dissipation rates. In CTX, helicity balance was demonstrated during formation using the measured gun flux, ignoring sheaths $\left(\mathrm{V}_{\mathrm{g}}>\mathrm{V}_{\mathrm{sh}}\right)$ and neglecting dissipation (small for $\left.\mathrm{t}<\tau_{\mathrm{K}}\right)^{1}$. In SSPX Gun flux and sheaths are not directly measured and lower gun impedance produces a lower gun voltage. From gun current thresholds for onset of gun voltage fluctuations, an inferred sheath voltage is $V_{\mathrm{sh}} \sim 100 \sim 150 \mathrm{~V}$. For plasmas sustained near threshold $\left(\mathrm{I}_{\mathrm{g}} \sim \mathrm{I}_{\mathrm{FC}}\right)$, when gun voltage is low, $\mathrm{V}_{\mathrm{g}}$ and $\mathrm{V}_{\mathrm{sh}}$ magnitudes are comparable and sheath helicity losses must be taken into account. For SSPX plasmas we use caltrans mhd equilibria fit to magnetic probe data to determine the captured gun flux and helicity content $\mathrm{K}^{2}$. From this analysis Thomson scattering profile measurements show improved confinement (reduced decay rate $\tau_{\mathrm{K}}$ ) with increasing electron temperature. This decay rate is compared with the helicity dissipation rate $\mathrm{K} / 2 \int(\mathrm{E} \cdot \mathrm{B}) \mathrm{dr}^{3}$ computed in caltrans using $\mathrm{E}=\eta \mathrm{J}$ and computing $\eta$ from the measured temperature profile. During slow field buildup with a long gun current pulse $(\delta \mathrm{t} \sim 3 \mathrm{~ms}) \mathrm{V}_{\mathrm{g}}$ is larger in the presence of long duration mhd fluctuations (e.g. $\mathrm{m} / \mathrm{n}=1,2)$. Although not yet confirmed by measurements of increasing $\mathrm{T}_{\mathrm{e}}$ during buildup, $\tau_{\mathrm{K}}$ increases during the pulse. Details of these results will be presented.

1. C.W. Barnes et al, Phys. Fluids 29 (1986) 3415

2. T. A. Casper, et al., 27th EPS Conference on Controlled Fusion and Plasma Physics, 12-16 June 2000, Budapest, Hungary.

This work was performed under the auspices of US DOE by the University of California Lawrence Livermore National Laboratory under Contract No, W-7405-ENG-48. 
MAGNETIZED TARGET FUSION DRIVEN BY PLASMA LINERS

Y C Francis Thio, Jason Cassibry ${ }^{1}$, Richard Eskridge, Ronald C. Kirkpatrick ${ }^{2}$, Charles E.

Knapp2, Michael Lee, Adam Martin, James Smith, S. T. Wu ${ }^{1}$

NASA Marshall Space Flight Center, Huntsville, Alabama, 35812, USA

francis.thio@msfc.nasa.gov (256) 544-7094

For practical applications of magnetized target fusion, standoff drivers to deliver the imploding momentum flux to the target plasma remotely are required. Quasispherically converging plasma jets have been proposed as standoff drivers for this purpose [1]. The concept involves the dynamic formation of a quasi-spherical plasma liner by the merging of plasma jets, and the use of the liner so formed to compress a spheromak or a field reversed configuration (FRC).

Theoretical analysis and computer modeling of the concept are presented. It is shown that, with the appropriate choice of the flow parameters in the liner and the target, the impact between the liner and the target plasma can be made to be shockless in the liner or to generate at most a very weak shock in the liner. At the same time, an extremely strong shock propagates through the target, producing the required preliminary heating of the target, thus setting the target at an initially high temperature (of a few 100 's eV) on an efficient adiabat for subsequent compression. The first converging and reflected shocks are analyzed using Richtmyer-Lazarus theory for spherically converging shocks. With the target temperature significantly raised by these first two shocks, subsequent shocks are relatively weak, and the compression is nearly acoustic. The acoustic phase of the compression has been modeled using Braginskii's transport coefficients for a magnetized plasma, allowance being made for losses due to bremsstrahlung and synchrotron radiation, and assuming ideal flux freezing in the target plasma. With the appropriate choice of operating parameters, the thermal losses are found to be relatively small. To check the overall flow dynamics, 3-D simulations of the merging of the jets and the compression of a target plasma are performed with the Los Alamos SPHINX code which uses a numerical scheme based upon the smoothed particle hydrodynamics (SPH) method. The detailed analysis and modeling of the implosion of a spheromak or an FRC by the plasma liner and of the Rayleigh-Taylor instabilities associated with this implosion remains to be done. Pilot experiments to study the interaction of the jets to form a 2-D cylindrical liner by the merging of 12 plasma jets launched by a circular ring of plasma guns are underway.

[1] Y. C. F. Thio, E. Panarella, R. C. Kirkpatrick, C. E. Knapp, F. Wysocki, G. R. Schmidt, "Magnetized Target Fusion in a Spheroidal Geometry with Standoff Drivers," Current Trends in International Fusion Research II, ed. E. Panarella, National Research Council Canada, Ottawa, Canada, 1999.

\footnotetext{
1 University of Alabama, Huntsville, AL, USA.

${ }^{2}$ Los Alamos National Laboratory, Los Alamos, NM, USA
} 


\title{
Plasma Liner Research for MTF at NASA Marshall Space Flight Center
}

\author{
Y C F Thio, R. Eskridge, M. Lee, A. Martin, J. Smith, \\ NASA Marshall Space Flight Center, Huntsville, AL 35812 \\ J. T. Cassibry, S. T. Wu, \\ University of Alabama in Huntsville \\ R. C. Kirkpatrick, C. E. Knapp, P. J. Turchi, \\ Los Alamos National Laboratory \\ J. F. Santarius \\ University of Wisconsin-Madison \\ francis.thio@imsfc.nasa.gov \\ (256) 5447094
}

The current research effort at NASA Marshall Space Flight Center (MSFC) in MTF is directed towards exploring the critical physics issues of potential embodiments of MTF for propulsion, especially standoff drivers involving plasma liners for MTF. There are several possible approaches for forming plasma liner's. One approach consists of using a spherical array of plasma jets to form a spherical plasma shell imploding towards the center of a magnetized plasma, a compact toroid (Figure 1). Current experimental plan and status to explore the physics of forming a 2-D plasma liner (shell) by merging plasma jets are described. A first-generation coaxial plasma guns (Mark-1) to launch the required plasma jets have been built and tested. Plasma jets have been launched reproducibly with a low jitter, and velocities in excess of 50 $\mathrm{km} / \mathrm{s}$ for the leading edge of the plasma jet. Some further refinements are being explored for the plasma gun. Successful completion of these single-gun tests will be foilowed by an experimental exploration of the problems of launching a multiple number of these jets simultaneously to form a cylindrical plasma liner.

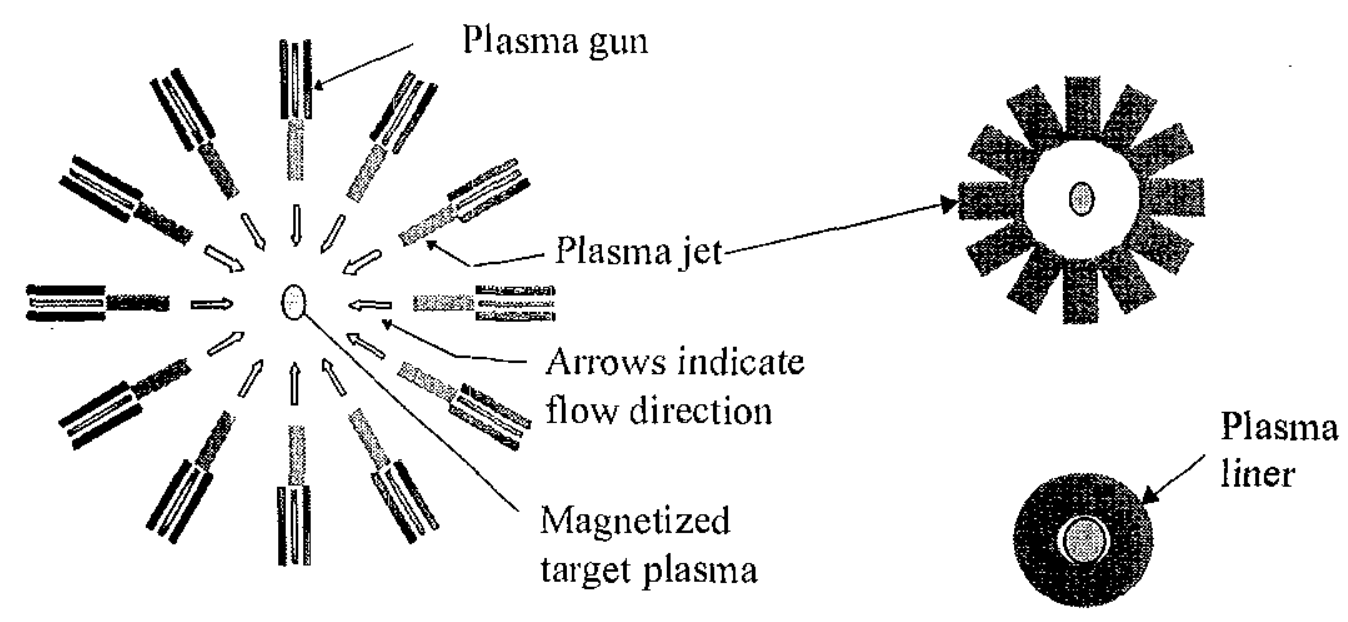

Figure 1a. A spherical array of plasma jets is launched towards a compact toroid at the center of a vessel.
Figure $1 \mathrm{~b}$. The jets merge to form a spheroidal shell imploding towards the center 


\title{
Conceptual Design of an MTF Space Propulsion System
}

\author{
Y. C. F. Thio and G. R. Schmidt \\ NASA Marshall Space Flight Center, Huntsville, Alabama \\ R. C. Kirkpatrick, P. J. Turchi \\ Los Alamos National Laboratory, Los Alamos, New Mexico
}

\begin{abstract}
Most fusion propulsion concepts that have been investigated in the past employ some form of inertial or magnetic confinement separately, and are encumbered by the need for advanced drivers (e.g. laser) or steady-state magnetic confinement systems (e.g.

superconductors) that have historically resulted in large, massive spacecraft designs. Here we present a comparatively new approach, Magnetized Target Fusion (MTF), which offers a nearerterm avenue for realizing the tremendous performance benefits of fusion propulsion. MTF attempts to combine the favorable attributes of both inertially and magnetically confined fusion to achieve both efficient and low-cost compressional plasma heating and energy confinement. The key advantage of MTF is its less demanding requirements for driver energy and power processing. Additional features include: 1) very low system masses and volumes, 2) relatively low waste heat, 3) substantial utilization of energy from product neutrons, 4) efficient, low peakpower drivers based on existing pulsed power technology, 5) very high $I_{s p}$, specific power and thrust, and 6) relatively affordable R\&D pathways. MTF overcomes many of the problems associated with traditional fusion techniques, thus making it particularly attractive for space applications. $I_{s p}$ greater than 50,000 seconds and specific powers greater than 20 kilowatts/kilogram appear feasible using relatively near-term pulse power and plasma gun technology.
\end{abstract}


DISCUS: A very high beta dipole disk-like equilibria physics experimental concept

\section{G.R. Tynan, S. Krasheninnikov, J. Slough}

Confinement of very high beta plasmas remains an important issue for magnetic fusion and space physics investigations. Recent theoretical studies of plasma equilibrium and stability in dipolar magnetic configurations have led to an important insight into high $\beta$ magnetic confinement [Krasheninnikov, Catto and Hazeltine, Phys, Rev. Lett. 82 (1999) 2689; Catto and Krasheninnikov, Phys. Lett. A 258 (1999) 153; Garnier, Kesner and Mauel, Phys. Plasmas, 5 (1999) 3431; Krasheninnikov, Catto and Hazeltine, Physics of Plasmas, 7 (2000) 1831 and references therein] - namely that strong magnetic field line curvature permits stable confinement of arbitrarily high local $\beta$ plasmas in a dipolar magnetic configuration. This result suggests that a route to the formation of a very high beta plasma $(\beta>>1)$ in a closed field line topology appropriate for magnetic fusion applications may be possible. In this paper we summarize these theoretical motivations and discuss the DISCUS experiment concept, which is a small-scale device which permits the study of very high beta disk-like plasma equilibria. Particular attention is given to plasma formation concepts which permit such a small-scale experiment to be performed. Similarities and differences with the $\beta<1$ LDX device, currently under construction at MIT, are discussed. 


\title{
High beta stability of magnetic dipole configurations
}

\author{
H. Vernon Wong, W. Horton, J. W. Van Dam \\ Institute for Fusion Studies, The University of Texas at Austin \\ Austin, Texas 78712 USA
}

December 19, 2001

For ideal MHD flute stability of magnetic dipole equilibria, the equilibrium scale length $\left(L_{p}\right)$ of the plasma pressure gradient is typically required to be comparable to or greater than the radius of curvature $(1 / \kappa)$ of the magnetic field line, $\kappa L_{p} \geq 1$. A sufficient condition for ideal MHD flute stability is $\left\langle\bar{\omega}_{\kappa}(\lambda)\right\rangle\left\langle\frac{5}{2} \bar{\omega}_{d}(\lambda)-\omega_{* p}\right\rangle>0$, where $\bar{\omega}_{\kappa}(\lambda)$ and $\bar{\omega}_{d}(\lambda)$ are the bounce averaged curvature and magnetic drift frequencies for particles with pitch angle $\lambda$, and $\omega_{* p}$ is the plasma diamagnetic frequency. The shape of the flux surfaces determines whether or not this inequality can be maintained with increasing plasma beta. If this inequality is violated at higher plasma beta, there will be a critical beta above which ballooning modes will be destabilised. Sharp pressure gradients $\left(\kappa L_{p}<1\right)$ may be possible if ideal MHD growth rates can be significantly reduced by finite Larmor effects and non-uniform $\mathbf{E} \times \mathbf{B}$ equilibrium flows (shear-flow). These non-ideal effects require $\frac{r_{L}^{2}}{\kappa L_{p}^{3}}>1$, where $r_{L}$ is the particle Larmor radius. However, plasma beta limits will be imposed by the necessity to avoid "drift reversals" and the onset of a compressional instability. Although dipole equilibria were specifically considered, these results may also be relevant to other high beta configurations. 

Heuristic model to explain the slow formation of spheromaks in SSPX without a globally
S. Woodruff, B.W.Stallard, H.S. McLean, D.N. mode
Lawrence Livermore Nation

Slow formation of Sational Laboratory, Califomia CA and the SSPX team in recent times of spheromaks by action of the

the $n=1$ mode in order to be formed common knowledge has been so well documented
slow formation with a coaxial spheromaks act

for which we can with a coaxial source (much liked. Not so. In SSPX ${ }^{*}$ waks actually need mode by raising choose to form spherom like the gun in CTX and WPe have observed current at around 200nt above a threshold. Without the $n=I$ mode, Or SWEX and CTCC) threshold, it seems, $\mathrm{kA}$ for $2 \mathrm{~ms}$, allowing We now have the ability to clatch on the $n=1$ would expect with, is related to the ejectis to explore thresholds mo clamp the injected impeded. Howev the programmed jection threshold (or stuffing accurately. This impression that the large amplitude gun of current to flux, for limit), and so one helicity ate thrown source is operating in a tage fluctuations, for the ejection to be conserve helicity from the source into the bursting mode, in which that give the high fluctuation, and form a spheromak by confinement region where finite chunks of increase from $I_{\text {ion }} / I_{\text {inj }}=0$ we observe current allaxation to the Taylor state . Doalesce, current above a thiesh $=0$ to 2 , in an almost amplification - we see the tote. During the even when the curesold. In shots that showear ramp and for as long as wal current in current path: current remains above this thres the $n=1$ mode, the formation is maintain from the source to thent needs to short in threshold. This can be explained seen to quit were to flow in a loop speromak ( $J_{X B}$ pushing gun in order to keep the hel by a change quit (no $J_{X B}$ in the loop around the spheromak andobs of plasma); howevericity flowing present, the current thrce). This is precisely wh return to the wall, ther, if the current (evidenced by a larent that flows from the wall what we observe: while this drive ought to to the mid-plane gape ramp in the toroidal will at the midplane gap increase $n=1$ mode is dies. While this gap, the gun-voltage fluctuld there), and when the curreases with time gaps in our und model is consistent with dations quit, and the configent path changes injector so that wanding. We plan to insta, further experiments arration flails and sheath voltage we can measure the helicity intobe to survey the prequired to fill helicity balane (which does not contributely injection rate $(\dot{K} \approx 2 V \psi$ plasma within the spheromak formaticulations based on to the helicity injection).

"The Suston points the way to higher dataset that show how the will present spheromak, formed confinement pred by a coaxial source (Mars Experiment, SSPX, is temperatures. spheromaks using a low of the spheromak, gun). SSPX has been a Im diameter $T_{\mathrm{e}} \sim 100 \mathrm{eV}, \mathrm{n}_{\mathrm{e}} \sim 1 \mathrm{e} 20 \mathrm{~m}^{-3}$ current source. Typ and to determine routes to assess the experiments although,$\beta_{e} \sim 4 \%$. SSPX is similarameters in SSPX ares to high field closely coupled sough differs substantially by vilar in design to preceding confinement region. 


\title{
Progress on the FRX-L FRC Plasma Injector at LANL for Magnetized Target Fusion
}

G. A. Wurden, P. N. Assmus, W. J. Feinup, T. P. Intrator, M. C. Langner, R. J. Maqueda, K. J. Scott, R. E. Siemon, E. M. Tejero, J. M. Taccetti, M. G. Tuszewski, Z. Wang, Los Alamos National Laboratory, Los Alamos, NM 87545 USA

\author{
C. Grabowski, S. K. Coffey, J. H. Degnan, D. Gale, E. L. Ruden, W. Sommars \\ Air Force Research Laboratory, Kirtland AFB, Albuquerque, NM USA
}

E-mail: wurden@lanl.gov

\begin{abstract}
The FRX-L Field Reversed Configuration plasma is now operational at Los Alamos National Laboratory. The goal of the project is to demonstrate the production of suitable FRC target plasmas for later MTF (Magnetized Target Fusion) implosion experiments which will first be carried out at the Air Force Research Laboratory in Albuquerque, New Mexico, in a few years' time. Expected plasma parameters in the $4 \mathrm{~cm}$ diameter, $30 \mathrm{~cm}$ long $\mathrm{FRC}$ are $\mathrm{n}_{\mathrm{e}} \sim 10^{17} \mathrm{~cm}^{-3}, \mathrm{~T} \sim 100-300 \mathrm{eV}$, at 4-5 Tesla fields, with a lifetime of $\sim 20$ microseconds. The system includes a $0.5 \mathrm{~T}$ bias field, $70 \mathrm{kV} 250 \mathrm{kHz}$ ringing pre-ionization, and a $1.5 \mathrm{MA}, 200 \mathrm{~kJ}$ main-thetacoil bank. Maxwell rail gap plasma switches are used to start the PI bank, the main theta coil bank, and to crowbar the main bank. Initial results using the first diagnostic set of excluded flux loops, B-dot probes, visible light diodes, a fiber-optically coupled gatedintensified visible spectrometer, and a 3.3 micron quadrature interferometer are presented. Future diagnostics include end-on bolometry, Thomson scattering, and a multi-chord fanned HeNe side-on interferometer. Multi-turn cusp and guide coils will be added later this year, to enable translation experiments into a cylindrical metal liner.
\end{abstract}




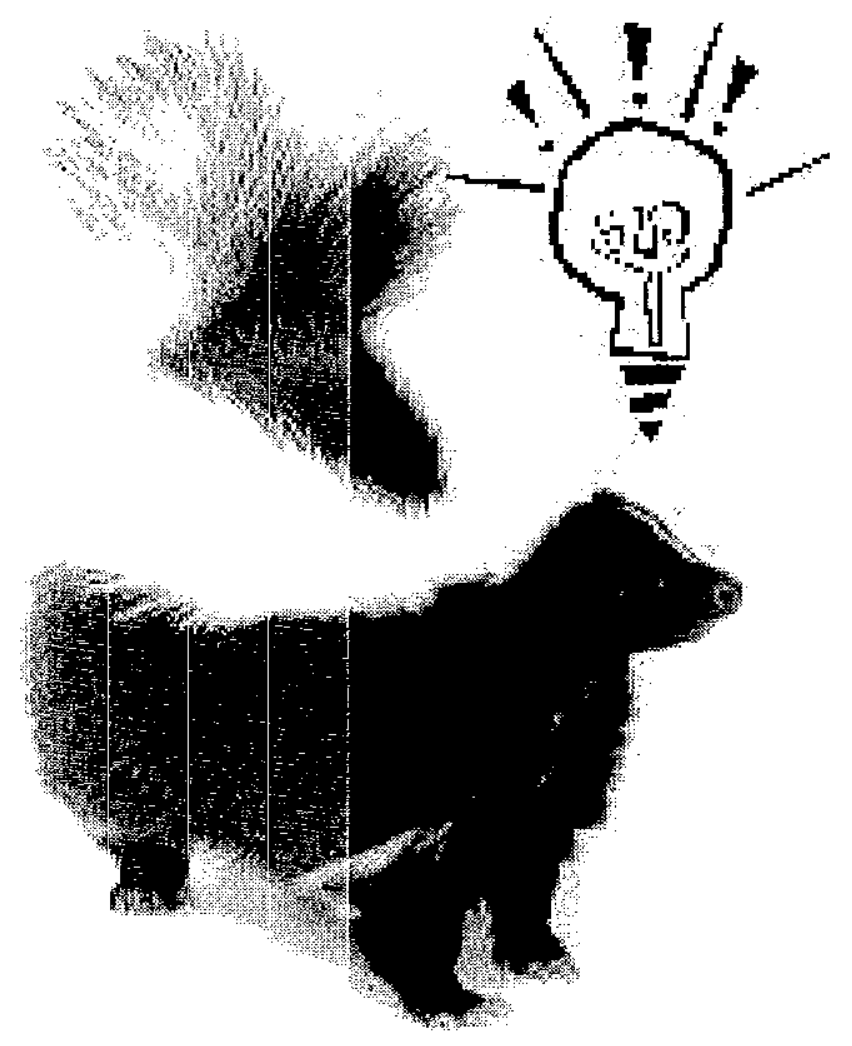


<smiles>[3H]</smiles><smiles>[12BH]</smiles>
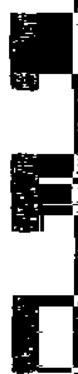

$3=$
$=$

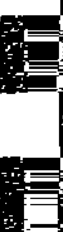




\title{
Plasma Heat Engine for High Q Aneutronic Fusion?
}

\author{
D. C. Barnes \\ Los Alamos National Laboratory
}

PROPOSED: Plasma oscillation provides a useful target for high $Q$ beam driven fusion.

\begin{abstract}
Consider p-B11. Spherical oscillation of B11 ions periodically produces an essentially pure ion plasma target. Striking this with a $\mathrm{p}$ beam at $650 \mathrm{keV}$ gives aneutronic fusion, but beam energy deposited by collisions is about 10 times the fusion yield. However, consider the oscillation as a heat engine. In a very modest $0.5-1.0 \mathrm{kV}$ well, the compressed $\mathrm{T}$ is $0.1-1.0 \mathrm{keV}$, while the expanded $\mathrm{T}$ is $0.1 \mathrm{eV}$ (radial compression 30-100). Thus, 0.999-0.9999 of the heat energy may be recovered. If only 0.99 were used for beam reacceleration, Q would becomes 10, near the maximum allowed energetically. Plasma engineering to allow this is proposed. Heat is removed from the expanded target by the extreme peaking at low $\mathrm{T}$ of ion-neutral collisions. This drives the energy of oscillations, producing a cyclic space charge. Beam injection during the proper phase then provides beam reacceleration from the oscillation energy. Such physics seem especially accessible in a system like PFX-I, but other possible embodiments are suggested.
\end{abstract}




\title{
Fusion via stagnation of self-excited counter-streaming toroidal flows in a highly rippled tokamak
}

\author{
Paul M. Bellan \\ Caltech
}

In conventional tokamak design one attempts to minimize toroidal field ripple because ripple causes adverse effects such as trapped particles and enhanced transport. Ripple minimization is achicved by using a large number of toroidal field coils. I wish to argue that it would be better not to use a large number of toroidal field coils and instead take advantage of a peculiar MHD pumping effect.

This pumping effect occurs because the curl of the JxB MHD force can be non-zero and so produce a torque on the plasma. Pressure gradients cannot balance a torque and so fluid flows result. The plasma current driven by an $\mathrm{OH}$ transformer will give rise to such a torque if the tokamak has ripple and this torque will drive counterstreaming toroidal plasma flows. These flows will be such as to convect plasma toroidally from regions of small minor diameter (ripple necks) to regions of large minor diameter (ripple bulges). Thus there will be a stagnation layer at the ripple bulges because flows will approach this layer from two adjacent ripple necks, one located toroidally clockwise from the ripple bulge, the other located toroidally counterclockwise. At the stagnation layer, the counterstreaming toroidal flows collide and their flow energy will be turned into heat. Since the flow velocity is of the order of the Alfven velocity calculated using the poloidal field at the ripple neck, the stagnation will produce ions with thermal energy corresponding to this poloidal Alfven velocity. Furthermore, because the flowing plasma is a flux conserver, it will convect poloidal flux from the ripple necks to the ripple bulges. The fluid pile-up at the ripple bulges will thus also cause a pile-up of poloidal field lines. The increased concentration of poloidal field lines at this pile-up will enhance the pinch force and so tend to reduce ripple bulgc. Thus, this pumping/convection effect will tend to reduce the overall ripple while heating the plasma. One can envision an equilibrium where fuel is ingested at the ripple necks, then is pumped toroidally to the ripple bulges where the stagnation of counterstreaming flows heats the plasma to fusion temperatures. In order to enhance ripple, the tokamak would have a very small number of toroidal field coils, say three. In operation, the pumping and flux pile-up effect would cause most of the ripple to go away so that the plasma will become nearly axisymmetric while being continuously heated via flow stagnation at the ripple bulges. Advantages of this scheme are a great reduction in cost due to a reduction in the number of toroidal field coils, a strong heating mechanism, and simplicity.

Evidence in support of this idea is three-fold:

1. Magnetic pumping is a well-known property of MHD and it is well established that this pumping tends to convect fluid from regions of constricted current diameter to regions of less constricted current diameter (this pumping had always been examined in the past for situations with zero helicity, e.g., a z-pinch with no guide field [1], but recently the author has shown that pumping should also occur in situations with magnetic helicity [2]).

2. Solar coronal loops tend to have uniform cross-section along their axis [3] and so can be thought of as being a toroidal segment of a rippled tokamak that has undergone this de-rippling process.

3. An experiment [4] on the ST tokamak at Princeton in which a purposely highly rippled toroidal field was imposed showed that the high ripple situation had significantly more energetic ions than the no-ripple situation. A four-fold increase in neutron production was observed for a $28 \%$ ripple (magnetic mountain) compared to a configuration with $1.8 \%$

An analytic model describing this pumping, flux convection, and heating process has been worked out for the solar coronal problem and application of this model to a fusion scenario will be discussed.

[1] P. M. Bellan, Phys. Rev. Letters, 69, 3515 (1992).

[2] P. M. Bellan, manuscript in preparation.

[3] J. A. Klimchuk, Solar Phys. 193, 53 (2000).

[4] W. Stodiek, K. Bol, H. Eubank, S. Von Goeler, and D. J. Grove, Proc. Fourth Intl. Conf. Plasma Phys. and Controlled Nuclear Fusion Res., (Madison, Wisconsin, June 17-23, 1971) paper IAEA-CN-28/C-10, Vol. 1, p. 465. 


\title{
Alfvenically Confined Plasmas
}

\author{
A.B. Hassam \\ University of Maryland
}

Large amplitude, torsional, standing Alfven waves could be used to confine plasmas for fusion. [1] The plasma is contained along the magnetic field as a result of inertial forces from the large Alfvenic accelerations. This is demonstrated by numerical simulation. Large instantaneous magnetic shear may provide MHD stability. Energy confinement would be limited by parallel electron transport, aided by Pastukhov factors. The Alfven waves would have to be driven at resonance inside the cavity, which makes for a large stored energy, though maybe manageable recirculating power. A "minor miracle" [2] might be needed to handle large, Ohmic, eddy losses in the cavity walls.

[1] A. B. Hassam, Comments in Plasma Physics and Controlled Fusion (1997) [2] as defined by Cris Barnes 


\title{
Muon Catalyzed Fusion Revisited
}

\author{
Russell M. Kulsrud \\ Princeton Plasma Physics Laboratory
}

It is possible to extend the number of fusions catalyzed by a single muon to as much as a thousand by going to low temperatures and utilizing cyclotron resonance to keep the muonic alpha particle going at high energies until the muon is stripped. However, because of low temperatures this can only be done by invoking a version of inertial confinement. The limit on the gain is set by the molecular formation rate. this has recently been shown to be much faster than has been expected and this increased rate enables a postive gain in fusion power divided by cost, Q, to reach values of at least 5 and perhaps more. I would like to discuss the feasibility of this approach. 


\title{
Fast Ignition and Heavy-Ion Indirect Drive
}

\author{
B.G. Logan \\ Lawrence Berkeley National Laboratory
}

\begin{abstract}
We assess heavy -ion accelerator beam requirements for indirect-drive compression of fuel for fast ignition (calculations by Debbie Callahan-Miller, LLNL). Single and double-ended hohlraums are studied with analytic scaling to estimate beam energy, pulse width, spot size, illumination solid angle, peak power required for fuel compression with two different hohlraum drive temperatures.
\end{abstract}




\title{
Carbon Free Energy Self Sufficiency by 2050 ? Possibly Via the Thorium $/{ }^{233}$ U Fission Fusion Hybrid*;
}

\author{
Wallace Manheimer, \\ Code 6707, Plasma Physics Division, NRL, \\ manheime@occ.nrl.navy.mil
}

Today, in the Washington DC suburbs, we buy gasoline for about $\$ 1.15$ per gallon; four years ago it was less than $\$ 1$, one year ago it was about $\$ 1.70$. Is there really an energy shortage, or is this all just the result of natural market fluctuations? While the short term may be murky, there are people who think long term requirements are clear (1). In a twenty first century world with $10^{10}$ people, all of whom will demand a middle class life style, and with a further constraint to minimize $\mathrm{CO}_{2}$ emissions, studies show that even with improvements of efficiency of $1 \% / \mathrm{yr}$, a tremendous improvement over half a century, the world will require an additional 10 Terrawatts of carbon free power by 2050 . There are not many technological options which can achieve this. Regarding nuclear power, estimates are that only about 60-300 TWyr are available in mined uranium, so breeding of some sort (or possibly uranium from sea water) would be essential. Regarding pure fusion, even its most optimistic proponents have not claimed they can achieve 10 TW by 2050. A possible scheme to achieve this power via the fission fusion hybrid is sketched out. We emphasize the thorium $/{ }^{233} \mathrm{U}$ cycle to avoid proliferation hazards, at least with the raw fuel. In the first fifteen or so years, we drop our plasma physics buckets where we are, and build a tokamak the size of JET. However it would run in steady state in DT and generate about 40 $\mathrm{MW}$ of fusion neutrons. With a breeding blanket, this would generate about $600 \mathrm{MW}$ of ${ }^{233} \mathrm{U}$ as well as breed the necessary tritium. We call this the scientific prototype $(2,3)$. In the next 15 year period, we would build the commercial prototype, a tokamak the size of ITER. The capital cost has been estimated at $\$ 10 \mathrm{~B}(\$ 0.6 \mathrm{~B} / \mathrm{yr}$ if amortized at $6 \%)$ and an operating cost of about $\$ 0.5 \mathrm{~B} / \mathrm{yr}$. This would generate $1.6 \mathrm{GW}$ of fusion neutrons, which would breed about $24 \mathrm{GW}$ of ${ }^{233} \mathrm{U}$ as well as the necessary tritium. In a conventional nuclear burner this would generate $8 \mathrm{GW}$ of electric power at a fuel cost of about $\$ 0.015 / \mathrm{kWhr}$. If successful, the world could build several hundred or a thousand of these in the next 15 years to generate multiterrawatts of carbon free power by 2050 . With a large number of these, the nuclear blanket engineering could be easier, because half could breed only tritium and half could breed only ${ }^{233} \mathrm{U}$. Of course for the world to accept nuclear power on this scale, it would have to be convinced that the proliferation and waste disposal problems are in hand. The use of a sub-critical mixture of ${ }^{233} \mathrm{U}$ and ${ }^{238} \mathrm{U}$ solves the proliferation problem, at least for the raw fuel. Solving the waste disposal problem would almost certainly require transmutation of the nuclear waste, preferably on the reactor site, but possible off site. It is possible a fusion reactor could help here as well.

*Work supported by ONR

1. M. Hoffert et al, Nature, 395, 881, 1998

2. W. Manheimer, Fusion Technology, 36, 1, 1999

3. W. Manheimer, Physics and Society, 29, \#3, 5, 2000 


\title{
Modular Systems as a Route to Economical Fusion Power
}

\author{
R. A. Nebel \\ Los Alamos National Laboratory \\ Los Alamos, New Mexico 87545
}

The single best predictor of economic performance for fusion systems is the mass power density (mpd). We will show that for conventional magnetic confinement fusion systems the largest leverage one has on the mpd is the wall load. Thus, first wall technology is a lot more important than the chosen confinement system for conventional fusion devices.

However, modular systems have an extra geometry.factor in the mpd which is the ratio of the device size to the chamber size. This extra factor allows modular systems to achieve high mpd without going to high wall loads. Electrostatic confinement systems are well suited to this approach since their confinement doesn't depend on the device size and the total power output scales inversely with the plasma radius. Some magnetic confinement devices (such as RFPs) may also be able to utilize this approach provided that the confinement permits it. Examples of both modular electrostatic systems and modular magnetic confinement systems will be presented. 


\title{
Nature's Constraints on Fusion: Where Might There be Some Wiggle Room (Thermonuclear and Non-Thermonuclear)?
}

\author{
L. John. Perkins \\ Lawrence Livermore National Laboratory \\ January 9, 2001
}

The fusion "skunkworks" process seeks concepts that:

(a) offer novel twist(s) on conventional fusion physics and/or fusion technology, and

(d) suggest the prospect for either reducing the size/cost/complexity of the fusion core and/or a novel application for fusion energy, and

(c) cannot be classified conveniently under the presently established divisions or research programs, and

(d) do not obviously violate the laws of physics.

In this paper, we will address the fundamental issues (laws of nature) that constrain the size, cost and complexity of conceivable fusion devices, both thermonuclear (magnetic, inertial, ...etc) and nonthermonuclear (non-Maxwellians, muon catalysis....etc). Examination of such constraints will focus our attention on possible wriggle-room for new fusion paradigms or for enhancing the attractiveness of our present concepts.

This work was performed under the auspices of the U.S. Department of Energy by the University of California, Lawrence Livermore National Laboratory under contract No. W-7405-Eng-48. 


\title{
The Kinetic Stabilizer/Tandem Mirror: Some Issues and Opportunities
}

\author{
R. F. Post \\ Lawrence Livermore National Laboratory
}

The Kinetic Stabilizer [1] is a method for the stabilization of axisymmetric open confinement systems, in particular tandem-mirror systems. The ability of open (mirror-based) systems to confine hot plasmas in near turbulence-free states has been demonstrated in experiments over the years. The most recent such demonstrations are those carried out in the axisymmetric Gas Dynamic Trap [2] and AMBAL [3] mirror experiments in Novosibirsk. In recent AMBAL experiments classical cross-field transport of a decaying hot plasma was observed, and in the GDT experiments transport was found to agree with classical rates. In GDT stabilization of MHD modes was achieved by unusual means, namely, by the interaction of the effluent plasma with the expanding magnetic field outside the mirrors, an effect predicted and analyzed by Ryutov [4]. In the GDT, since it operates in a high-density, collisional, plasma regime, the plasma leaking through the mirrors, although much lower in density than the confined plasma, is high-enough in density to MHD-stabilize the interior plasma, at beta values of 30 percent. This approach will not, however, be effective with plasmas having the densities, temperatures, and low-collisionality needed for tandem-mirror fusion systems. The Kinetic Stabilizer, based on an earlier concept, the Kinetic Tandem [5], employs ion sources, located far out in the expanding field outside the outer mirror of an axisymmetric tandem mirror system. These ion sources are aimed at small angles to the field lines so that the injected ions are compressed, stagnated, and reflected upon moving up the magnetic gradient, forming a localized plasma peak in a region of strong positive magnetic curvature. This plasma peak thus performs the same stabilizing function as the effluent plasma in the GDT. Calculations to be presented show that the beam power required to stabilize both the contained central plasma and the endcell plasmas in the tandem-mirror is small compared to the fusion power. Not only is the stabilizer beam power small, but also the use of axisymmetric field coils will allow the achievement of very high fields in the end cells. High fields and axisymmetry shrink the end-cell volume, at the same time increasing the central-cell mirror ratio. These effects, taking together, allow a return to the original tandem mirror idea of Dimov/Fowler/Logan, that is, achieving the required plugging potentials solely by increasing the plasma density in the end-cells relative to that of the central-cell. Now axisymmetric, the tandem mirror should be both simpler and better in its confinement properties than non-axisymmetric tandem mirrors with thermal barriers. The paper will review the MHD-stability calculations and some of the special plasma physics issues that have arisen to date in studying the Kinetic Stabilizer concept.

*Work performed under the auspices of the U. S. Department of Energy by the University of California Lawrence Livermore National Laboratory under contract W-7405-ENG-48.

1) R. F. Post, Transactions of Fusion Technology, 39, 25 (2001)

2) P. A. Bagryansky, et. al., Trans. of Fusion Technology, 35, 79 (1999)

3) T. D. Akhmetov, V. S. Belkin, V. I. Davydenko, G. I. Dimov, et. al., $28^{\text {th }}$ EPS Conference on Controlled Fusion and Plasma Physics, Madeira, Funchal, Portugal, 18-22 June, 2001.

4) D. D. Ryutov, Proceedings of the Course and Workshop, Varenna, Italy, Vol II, 791 (1987)

5) R. F. Post, Trans. of Fusion Technology, 35, 40 (1999) 


\title{
Proliferation-Proof Fusion Power
}

\author{
J.F. Santarius, G.L. Kulcinski, and L.A. El-Guebaly \\ University of Wisconsin \\ 1500 Engineering Drive \\ Madison, WI 53705 \\ santarius@engr.wisc.edu \\ 608-263-1694
}

Few problems trouble today's world as greatly as the proliferation of nuclear weapons. This talk will address the question of whether an advanced-fuel fusion power plant can be designed to be proliferation proof. To achieve this solution, a magnetic fusion power plant would be operated in a very high power density mode that would be extremely difficult to modify for use with the high-neutron fusion fuels that could be used to breed fissile fuel for nuclear weapons. That is, production of $>1 \mathrm{~kg} / \mathrm{yr}$ of weapons-grade fissile fuel would be extremely difficult. We anticipate that the most likely solution will burn the advanced fuel combination of deuterium and helium-3 (D- $\left.{ }^{3} \mathrm{He}\right)$. The high power density would derive, for example, from using (1) a high-_ field-reversed configuration (FRC), (2) a strong magnetic field, and (3) direct conversion of charged-particle energy. The proliferation resistance would stem from (1) high power density coupled with a small radius, thus leaving minimal space for radiation shielding, (2) low neutron wall loading, greatly increasing the time required to produce significant amounts of fissile fuels, (3) use of energy conversion technologies, such as direct conversion, that match advanced fuels well but are poorly suited to D-T fuel, plus (4) full lifetime first wall and shield modules - stemming from the low neutron wall load and making replacement very difficult. Burning an advanced fuel would require substantial, continued progress in plasma physics, particularly better plasma energy confinement and development of the FRC or another suitable innovative confinement concept. The power plant would be optimized for non-proliferation, so the cost of electricity (COE) would likely be somewhat higher than for a power plant optimized for minimum COE. The key objective would be to design a turnkey fusion power plant so difficult to modify for use with high-neutron fusion fuels that it could be exported, yet be easily monitored to prevent nuclear proliferation. 